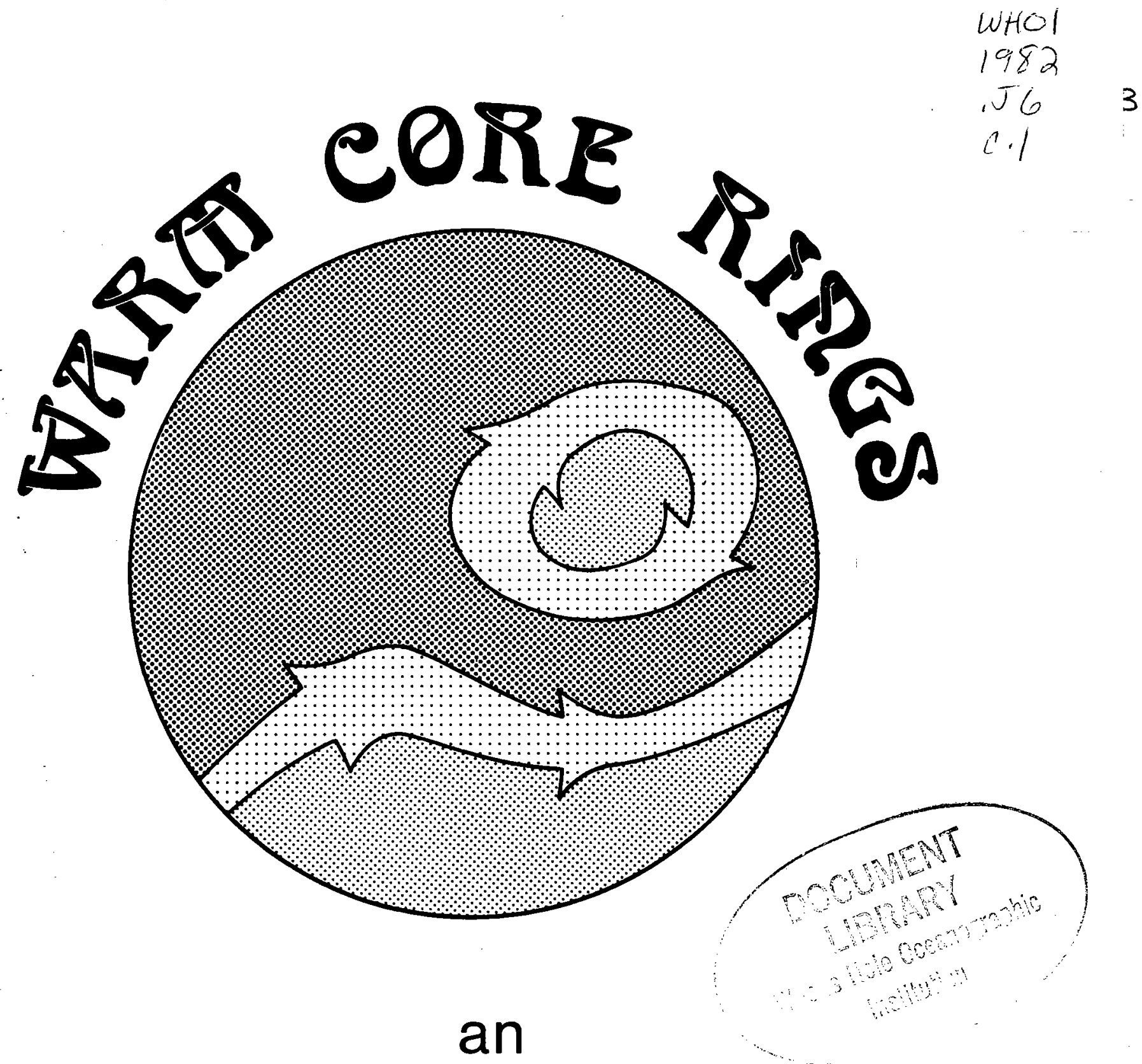

\title{
Interdisciplinary Study of Warm Core Ring Physics, Chemistry and Biology
}

ישטומתוש CRUISE REPORTS

\section{RV/ ENDEAVOR - RV/KNORR - RV/OCEANUS APRIL 1982}


CRUISE REPORT

ENDEAVOR CRUISE NO. $83,4 / 17 / 82-5 / 8 / 82$

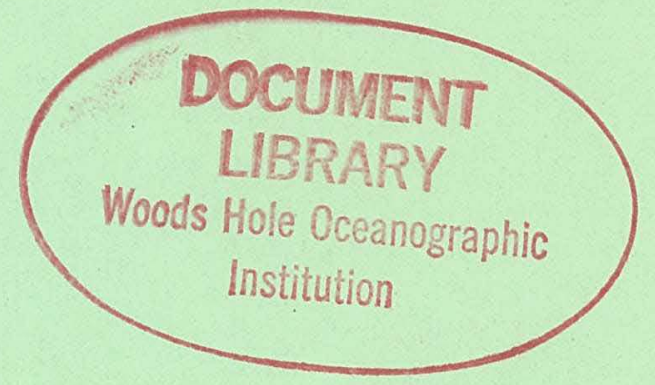

Terrence M. Joyce

Woods Hole Oceanographic Institution

Woods Hole, Massachusetts

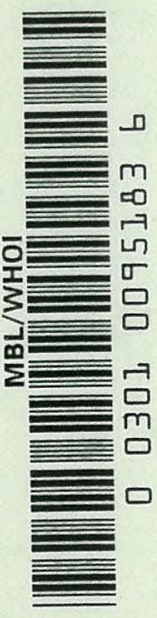


WAFM COFE FINGS CRUISE FEFOFT: ENDEAVOF BS

This report will summarize the shipboard activity aboard the

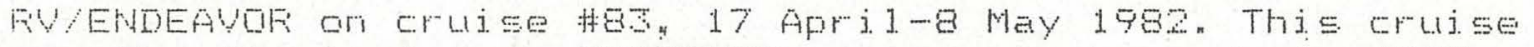
was the first of four ENDEAVOF cruses to be made in dogz as Fart of the Werm Core Fings (wCF) experiment. The report will be presented in a topical maner following an overall cruise narrative" It. js meant to serve as an informative and useful. document to those investigators within the WCF program - not as a puti.jeation for ci.rculation to the general scientific comimurity

\section{CFUISE NAFFATIVE}

The ENDEAVGF departed from Woods Hole shortly before 11 am EST (1600Z) on 17 April with a scientific party of 15 and $T$. Joyce as chief scientist. A list of the scientists and their irstitutional affiliations can be fourd in a later section. A meeting of the chief scientists aboard the ENDEAvOF wes held prior to departure to plen on the strategy to be used in ring Eejection. A mewly forming rjng, 82-D, was to be quickly Eurveyed by ENDEAVOR, and if suitably detatched from the GuIf Strem the other three ships ifNOFF, OCEANUS, E ALEATFOSS IV) would converge on that $r i n g$ as a study site. If BZ-D was not a viable rimg, the NSF-funded ships would study the backup ring 82-E. The ALEATFDSS IU group was not certain whether the interestes of the vational Marine Fisheries would be in the study of a ring which was already west of Georges Eant- (i.en a2-5). The plan for ENDEAVOF was to steam directly to the center of $82-{ }^{-}{ }_{5}$ based upon sateli ite data, mate our first test station in the enter" followed by a quicl: XET survey.

We tegan tating XET's at 2 hourly interval at obooz on day 108 (19 April) ard arrived at the wenter location at $1650 z$ that day, The remote ensing people were quite accurate in their estimate of ring center positiom at the site of our

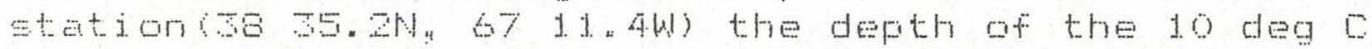
isotherm was 763 m in contrast to 200 m outside: and the ring currents, which reached $\Xi$ tots to the ww of the center. had decreased to orily us ltsen The CTD-az cast in the center revealed no 18 deg $c$ core of Sargased Sea water but rather water of Guif stream origin. Further maping with XET" a and our doppler current profiler reveded the ring 82-D to be. in large part. attached to the Buff stream In order to avoid the rapiddy chenging condjeians encountered in aur previous

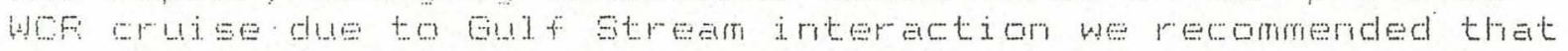
ring Bz-E be selected for study After a consensus decision was reached that WCF wort at az-D be stopoed the ENDEAVOF derarted to the west at $1618 \mathrm{z}$, day 109 , for $\mathrm{Qz}-\mathrm{E}$.

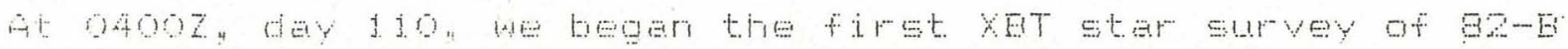

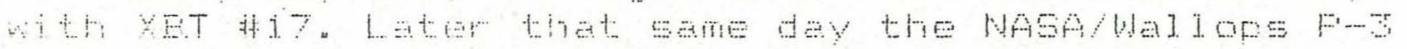

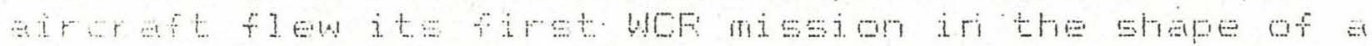

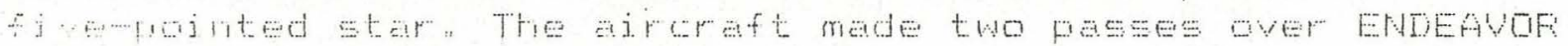


while we were on the second leg of our star on a course of o19. At the end of this leg. the ship was stopped for a shajlow E[FS (Eig-Optical. Frofiling System) cast at the rorthern-mast paint of the star. At $1100 z$, day 111. the Survey was completed, and ENDEAVDF headed to 38 . $20,70,19 w$, Where the first cast of our asterist-shaped CTD-OZ/EDFS serj.es was to be made.

The fjist Erass-ring transect began at $1446 z$, day 111 , with station a and ended at $1100 z$, day 11 . station consisted of a deep (to the bottom) CTD cast and a sha]low (to 2oo m) cast with the EOFS. The station spacing far this and the subsequent two transects was 10 nmi The second $r$ ing trameect was from 1500z, day 113 (station 14 ) to 1457t, day 115 (sta 24). The firal transect started at 1950Z, day 11. completion of sta J5. During the first transect the LOFAM-C drjfter was Ialmohed near the ring center"

The LOFAN drifter bas a compound drifter which consisted of a WHOI radar refjectirg buoy under which a LDEO sedjment trap was susperided at the end of 100 m of line. which acted as a distributed drogue and located the sediment trap at the base of the euptictic zorie. This drifter was tethered to a Fismas LOFAN-C bugy that intermal. y recorded its LQFAN pos tian and telemetered the results at a fined schedule to the ship. The

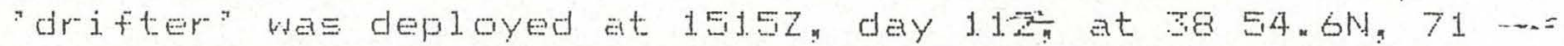
16.7w. Durimg the course of our asterist pattern the ring drifted to the west. New updates of the satelijte-inferred center location enebled tre station pattern to be modified s]ights $y \leq 0$ that each of the three transects was centered on the drifting rirg. while ENDEAVDF was within range of the Lofifid $t e$ ] emetry af the drifter we were able to manitar the drifter tracl: as it roteted at a redils of $5-9$ rim about: the rj. II CEnter.

While on stetion 19 the signel wes lost from the CTD figh as we were haulirig in the instrument. It was later discovered thet the new piece of approsimately 7000 m at conducting cable on the GTD wirch was shorted between the certer conductor and the armored shield. Thereafter al s stator worla had to be done on the aft winctin The ehallow EOFS Caste were not affected, but time was lost duririg the deEp CTD casts becolse thje wimch djd not Ievel-wind properly. Approsimately 15 houre of etetion time wes 10 t. beceuse of level windirg

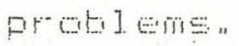

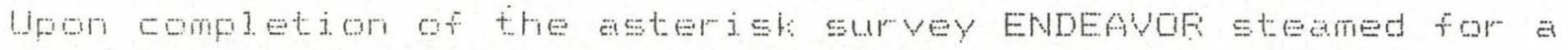
dead-rechoned estimete of the drifter position. without reg virg any further telemetry LQOFAN twarsmission had in fect etopped due to low battery voltege) the drifter wes

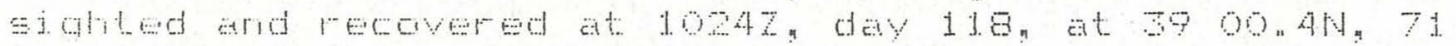

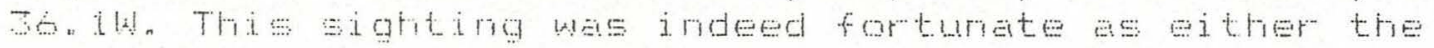


ENDEAVGR 83

drifter or a considerable amount of ship time could have been Iost! Three of the four fUC ededinent traps were recovered as we1]. Fol lowing this recovery ENDEAVOF steamed another hour to join rNDFF and OCEANus for the first muti-ship experiment j. I the ring centier".

At this point the wind was picting up and it was decided not to deploy one of our drifters in the ring center" Instead the three ships were to maneuver" relative to a geographical location. Upon reaching the location for the experiment ENDEAWOF hOVE to near NWOFF fOr a HEEP CTD cast at $3854.5 N$, 71 42.4W, 11367. After this, a BOFS cast was made fallowed by a combiried BOFS/XET survey in the shape of an $x$. This was centered 9 rim SW of the kNofi with the site of the deep cast as the center of the pattern and the kNofing position as the endpoint of ane of the two orthogonal legs. Along each leg. XET" E were launched at ten minute intervals. At the end of each leg. a BuFs cast was made. Due to worsening weather we caricelled the final EOFG (ass ( patterm. We finished our work: in the fest multi-ship experiment at 2340 y day 118.

The chief scientists conferred on VHF and decided that the second we experiment was to be in the Nw quandrant of the ring at the frontal boundary. It was also decided that each of the vesesels would head to the Nw along a different tract: so as to obtain a good spatial picture of the ring structure in the region of the Znd MS experiment. At 1222Z, day 119 a rejuvanated LOFAN drjfter was 1 aunched at $3921.4 \mathrm{~N}, 71.54$. $5 \mathrm{~W}$. MNOFF was to spend nost of her time during the course of the day "hove to" near the drifter location while oceanus and ENDEAVOF were to wort: relative to the drifter. The drifter consisted of the Lofiand buoy tethered to a large, radar transpondirg. plarit-on-edge buoy which had a window shade drogue at a depth of 100 m. The drifter was recovered at the end of the experiment $3917.7 N_{1} 71.12 .5 W$ at $1230 z$ after a drift of nearly 24 hours. During the Znd MS experiment the CTD package was towed at a speed of 2 kts while the j.netrument was rajeed and bowered repeatedly in a yo-yo manmer. This "tom-yo" was switched from the starboard I-frame to the aft a frame to prevent the cable from chafing againet the huld of the ship. At the corners of the bow-tie-miped pattern. BOFs casts were made. The maximum range to the ractar trangponding drifter during the survey wes 3 nom. The second NASA miscion was fiown at this time under joproving weather and cleering slates A pectege conteining two boxes of floppy dj.sts was air dropped to Fusmith on the ENDEAVGF to hedp al levate a eevere elortage of diste for the biomoptical proorem. one of the boxes flodded but the other one wes later put to good use. An AXET pattern was made to map out the fromtel structure in the sub-eurtace thermel fiejd as wej. as the surface color" fluorescencen and irifrered temper ature. We ardicipate thesedate to be of great vedue to the sower 
movirg ships.

As the ENDEAVDF departed far the Sargasso Sea to begin a section to the west of the ring, hourly X.ET"s were taken through the ring center to the southwest. At the sw edge of B2-E, we turned to a southerly heading shooting 2-hrly XET"s. unti]. the GuIf Stream was crossed. A CTD/BOFS section was begur with sta $38,3537.3 \mathrm{~N}, 7209.6 \mathrm{~W}$ at $1309 \mathrm{z}$ on day 121. The section consisted of 10 stations across the Gul f Stream and Slope Water with 20 nom spacing. The section was completed at $150 \mathrm{G}$, day 123 at which time ENDEAVOF returned to 82-B for the second XET star survey.

The secand and final XET star began with XET \#101 at $1900 Z$, dey 125 arid ended with XET 148 at 2000 on day 125 . The fourth leg of the star was brotien and a final. CTD/BOFS station (\#48) was made in the ring center at ożiz on day 125. This second star pattern was larger than the first one in order to get outside the region of strong ring currents to the slower moving Slope Water surrounding the ring.

The final. CTD/EOFG section wes begun in the Sargasso sea with sta 49 et 09172 on day 126. This section consisted of only 7 stations spariring the Gulf Stream and Slope Water along 70 . W with 50 mm spacing. The time lost due to problems with the level-wind on the aft winch required us to cut out three stations along this line. We were able to begin our sectiofm j.n the Sargasso and fid.led our remaining nutrient sample bottles in western boundary undercurrent water flowing to the west along the continenta]. rise. This "upstream" section to the east of $92-E$ ended with sta 55 at $0324 z$, day 128. We then steamed to koods Hole arriving around $1430 \mathrm{z}$ ater that same day (a May). The unloading of the vessel was accomplished in about three hours and the ENDEAVGF sas well as the scientific Farty) headed for home.

A chronological. Summary or event log for the cruise EN-BS can be found in Table 1 .

\section{CTD/O2 FFOEFAM}

A total of 55 caste were made with two djfferent CTD under nater unite during the cruise. Table 2 contains a summery, or extract, from the event log of the CTD stations. Injtialy CTD\#7 was modified to djojtize and transmit data from a two-bannel transmiseometer provided by I. Bishop from LDEO. On its first cast. station 2" the unit functioned on the way dow to the bottom but as it was coming back to the surfacen the sugnd from the transmiseometer dropped to velues much below troes om the down tracen ffter discussion over the radio wjth Bistrop, it was decided to remove the unit.

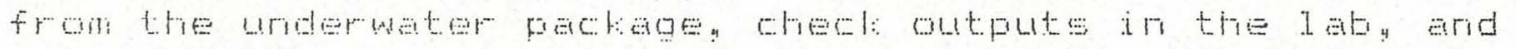
set it aside co that j. could be shipped bact to areoon for 
repair (jt was brand new and sti.l I under warranty). Eoth CTD fish included Beclum dissolved oxygen sensors and a 24 Eott1e, 1 "2 1iter Generaj. Gceanics Fosette. We experienced problems with the o2 sensor and had to replace it in CTDA7 after sta 11. Other problems encountered were : * no water eamples on sta 19 (Nistin bottle valves were left operis)

* a short in the conducting wire on sta 19 which allowed us to take only four water samples

* a switch to CTD将 after sta 26 because battery voltage on CTD\#7 had dropped too J. OW. CTD\#7 was again used during and after the multi-cast tow-yo sta 37

* CTD\#7. Was replaced by CTD\#s after sta 51, al though the 02 cEll from \$7 was LISed

Some of the 02 sensor problems arose because CTD\#7 drew excessive current from the dect: unit and did not charge its j. nternal. battery. With low battery voltage, the oxygen cell became noisy" A few stations have perhaps irrecoverable continuous oxygen data.

The three CTD sections through WCF $82-\mathrm{E}$ were taten at $10 \mathrm{~mm}$ intervals and consisted of eleven stas/section. Date from these stations (s-Sb) should provide the best available CTD coverage to date of a warm core ring. A full set of nutrient samples were collected and frozen for analysis ashore. In order to limit the number of semples to managable (e funded) level, the mutrient sampling was selective at. stations other than s-Sb. At each station we obtajned as many as 20 bottle samples for salinity ard oxygen analysis at sea. an two of the wistin bottle frames reversjing thermameters were mounted in or cler to chect: the temperature calibration of the CTD L. $^{3}$

Ar experimental. platinum temperature frobe was added to CTD\#7. It is menufactured by Fosemount with specification for a faster time response than the standard Fosemount sensor " $A$ cursory examination of the data indicated that it was at lecte 100 miliseconds faster than the standard platinum sensor, its celibration was stablen and its noise level low and of the order of 1 mdeg $C$. We are hopeful that this new sensor wil 11 a a long way towards bridging the gap between the slow temperature and fast conductivity sensors which causes problems when salinity j.s calculated. 


\section{XET FFOGFIAN}

A11 177 XET's taten on the cruise were of the T7 type, and all were digitally recorded on a Bathysystems recorder. "Data files of selected j.sotherms were transmitted via SDAT (see communications) to other WCF investigators in a quasi-real-time basis. A table of XET positions (Table 3 ) can be trot:en down into several tasks:

$\begin{array}{ll}\text { 1) } & 1-15 \\ \text { 2) } & 16-47 \\ \text { 3) } & 48,49 \\ \text { 4) } & 50-77 \\ \text { 5) } & 78-84 \\ \text { 6) } & 85-98 \\ \text { 7) } 101-148 \\ \text { 8) } 149-162 \\ \text { 7) } 16 .-177\end{array}$

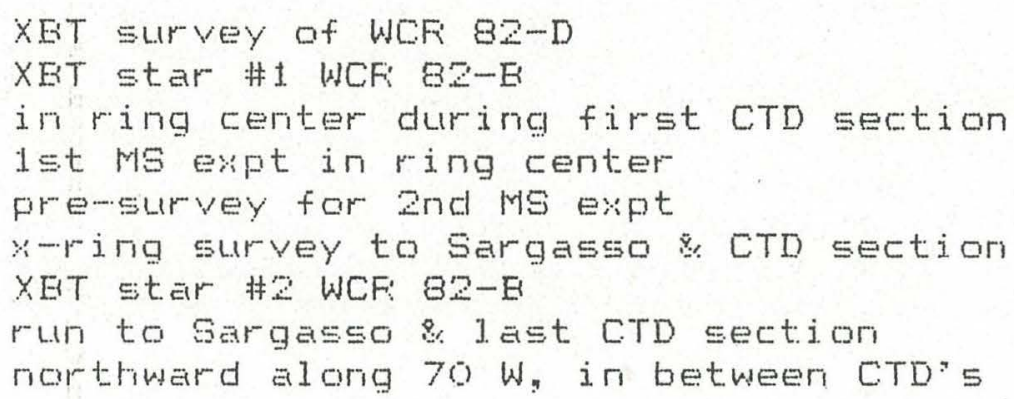

\section{ACQUSTIE FFOFILING SYSTEM (AFOC)}

A doppler acoustic current profiling system was operating during the entire crujse logging data onto 9T digjtal tapes and giving real-time printouts of currents to aid in our underway mapping program. This system was eseentially the eame as that on the previous WCF eruise =ENDEAVOF-74) with $=$ the addjtion of several. new underway sensors. A SAIL module was buj.t 50 the we could monitor the temperature from a pl. atjmum thermometer mounted in the transducer wel. d. and belonging to UFI. This provided us with a digital record (every so seconds) of the "buctiet" temperature. At the same time deta from a WHOI-built themosalingoraph was recorded. This unit wes constructed following an 0 bu design and consisted of GEAEIFID sensors far conductivity and temperature. While underway we collected seawater samples from the uncontaminated seawater line for selinity determination and later calibration of the thermo-salinograph salts. The unit functioned with only minor attention for the entire cruise. The conductivity cell drifted, however, and it will hot be possible to calibrate the system with a single offeet for the entire cruise. A total of $45,1200 \mathrm{ft} .9 \mathrm{~T}$ digitel tapes were filled with data while at sean some of the real-time printout was transmitted to SDAT and Miami. along with the corresponding XET or CTD data.

\section{BTO-OFTICAL MEAGUFEMENTS}

F. Gmith and his comorlems collected data for the purpose of descriptively and predictivly modeling the photoenviroment af a MLF This efrort should provide a basis for the dynamicel modeling of phytoplarkton in ringe and their environs as wed. as provjding complementary surface data for 
aircraft and satelitite remote serising research. This program was furided by NASA, which al 00 supported the AFQC wart: described above and the aircraft studies.

The along-tract: date recorded once per minute, included:

1) time arid LOFAN-C: location

2) sea surface temperature and conductivity

$3)$ air temperature" wind speed : direction

4) total solar j.radiance (0.3-3. o microns)

5) Liltra-violet irradiance (320-360 nm)

6) fluorescence measurements of chlor, phyco, \& fucazanthin

7) hourly discrete samples for extracted chlorophyl 1

Al. I Eampling was from the same uncontaminated seawater. 1.ine used for the above AFOC measurements.

Vertical profiles were made with the Bio Qptical Frofiling System (EOFS) at each of the 55 stations to a maximum depth of $200 \mathrm{~m}$. In addition to conductivity, temperature, and pressure, measurements were made of upwelling and downwelling irradjance in 12 bands of the vissible spectrum, downweling irradiance at 380 nom upwelling radiance at four wavelengths carresponding to those on the czCS visible channels, and fluorescence. The pressure case which leated on the first WCFi cruise (EN-74), had been replaced. Continuous data were collected at all of the casts although some data loss was experienced in the ear ly stations. Finally, a 121.7 liter Fiosette (loaned from WHOI) was attiched to the EDFS anad discrete bottle samples were collected for clorophylj. determination and the study of phytoplantoton species composition.

\section{FHYTUFLANKTUN SFECIES CONFGSITION BTANDING CFOF}

F. El actwelder collected 1 iter water samples from the BOFG Fosette for an asesement of phytoplantiton species composition and standing crop in 82-E and its surrounding weters. Five depthe were routinely sampled $6150 \mathrm{~m}, 75 \mathrm{~m}, 40 \mathrm{~m}$, 2om, surface). A total of over soo samples were filtered at sea using a four port Gelman filtration setup and the

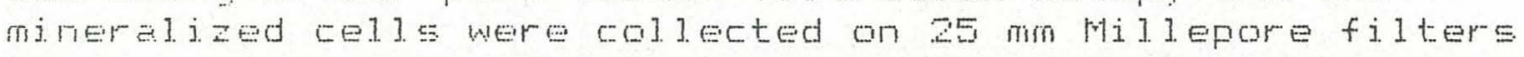
having a pore size of o. 8 microns. Nine underway surface samples were tatem as a test of the feasibility of simul teneous chioroptylifcommuity structure assessment. An evaluation of thise technique will be made ashore.

During each of the three ring transects a full rine bottle profi] down to $150 m$ was talen when ENDEAVOF was in the center of the ring similar profiles were also made at sciected stations jn the high velocity region, Slope water" Gulf Gtream, and Sargaseo bea. Microscopic examination of. samples from several ring transect stetions wes als a acoompl jehed on board ship. 
Fi. Evans was in cherge of U. Miamj. LQFiAN-C drifter operations during the cruige. During the first deployment rear the center of Bz-E, the Lofial drifter was tethered at the 1 ifting harress to a MHOI radar buoy. A tag lirie with a float was al 50 attached at. this point. A high gain (Sdb) vertical. antenra was used for both LofiAN reception and data telemetry. LGFAN reception was excellent and compared favorably with the regular antenma/coupler on the ship. All mooring gear for the deployment was attached to the radar buoy. A 2 m section of chain was shactiled to the buoy. Under this came a $10 \mathrm{~m}$ section of coated wire followed by $100 \mathrm{~m}$ of hydro wire and finally 20 m of nylor. On the first deployment two distributed temper ature sensors were attached to the mooring I ine: one neer the surface placed in-line between the chain and coated wire and the second between the hydro wire and the nylor. The LDGC sediment trap was placed near the bottomy $5 \mathrm{~m}$ above a bo pound lead wejght. A wire clamp secured the trap to the rylon. This letter was a mistate as the wire clamp chafed through the nylon with subsequent loss of the lead weight: The trap was seved by a lonot that was placed in the nylon as a safety. Three of the four traps were recovered. How the fourth was lost is a mystery since the rubber strap securimg it to the frame was stil. in place.

Thie telemetry range varied from 1 eses thein 23 rim when the wixad was brist and the ajo dry to greater than b5 nm with 1 ight wirdse and high humidity. As the telemetry data westeceived the drj.fter location was plotted. After a deployment of sia days the drifter was recovered. As noted in the cruise narrative! the recovery was a combination of good luct and (some) plamming. At the time that the CTD asterisl pettern wes completed we had received no drifter telemetry for 14 hours. Eased upon the recent drift history a dead recloried position was estimeted and the ship vectored to an intercept position. As the distance decreased the telenetry signal was again received, but the data were all nulls (low battery valtage). Leing the strength of the telemetry signal together with astute visjor, the flashing light was sighted through the dewris mist arici the drifter recovered with $2+$ meters of

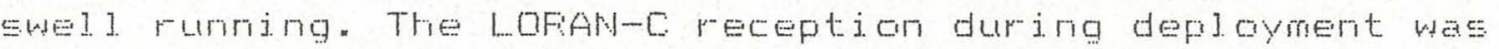
good and the resulting trajectory obtained from the magrietic: tape record was smoth with only a few recorded frames having bit ererson

Aे eEond deplayment was made durjng the 2nd MS experiment. Duriro this time the Lofifd drifter was tethered to the radar transpondjng plant:-on-edge buoy under which a window shade drogue we suspended at. the end of 100 in of hydro wire. No sedjment trape or temperature sensors were ine luded dur jug

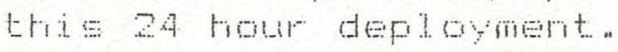


As on the first WCF cruise last year (EN-7A), an LSI computer with a winchester dist was the intermediary device for tent and data communication with other vessels and ashore via the ATG gatellite commuication link. Dater either went out onto Telenaj. I to a user group SDAT, or went directly to the U. Mi emi, FiSMAS VAX computer. The primery use for the latter route was for: transmittal. of all of the underway biomoptical observations to Miami where shore-tased processing of the data tool place, with some results transmitted bact: to ENDEAVOF: transmittal to the ENDEAVOF of thermal IF satelitite data and processed results on frontal boundaries and ring center position: and sending of XBT, CTD, and AFOC data from ENDEAVOF to Miami. "The Telemail. 1.j.nt was used for data transferral to other vessels and to NASA so that they could plan their two ajrcraft missions. We were also able to receive some XET data and text from the OCEANUS. The ALEATFOSS IV and fNOFFi were noticably silent on Telemail. We also received telecopy data from UFI during the voice schedule. This was set for three, one hour periods during the day (starting at $1200 z, 1700 z$, and $2200 z$ ). The $2200 z$ schedule was arranged through F. Eden exclusively for Warm Core fings. Eecause of this extra hour and due to the frequent use of VHF radjo when the vessels were operating in close proximity " the HF Eidetand was not used except for communication with Wallops Flot on Wallops Island. Unlit:e the first WCF crujsen= the ATS hardware, once worling, remained ful Iy functional for the entire cruise without constant maintenance. 
The members of the ecientifje party aboard cruise 8 S of the FiY ENDEAUOF, their institutional affiliation and principal tasts are listed below.

\begin{tabular}{|c|c|c|}
\hline Terrence Joyce & WHOI & Ch. Scientist \\
\hline Faymond Schmitt & WHOI & WCF: physics \\
\hline Marvel Stalcup & WHOI & hydro, drifters \\
\hline Fotert Mcdevitt & WHOI & CTD hardware \\
\hline Jane Duriworth & WHO & CTD processing \\
\hline Cileo Zarii & WHO & AFOC hardware \\
\hline Lorraine Earbour & WH & AFUC processing \\
\hline Cyntria Tyrian & WH & Autosal, CTD \\
\hline Fiaymond Smith & UCSE & Bio-optics \\
\hline Jeffrey star & Scripps & Eid-ops holwre \\
\hline Charleen Epstein & UCSE & Eio-ops proces. \\
\hline Fatricia El] actwel wer & Nova U. & Fiytopl ant:ton \\
\hline Fobert Evans & FSMMAS, Mi ami. & drifter, commo \\
\hline Stanford Hooler & FISMAS & drifter: plots \\
\hline Dävid NeJson & UF:I & marine tech \\
\hline
\end{tabular}

ACFNOWIEEDGEMENTS

The scientific party aboard the ENDEAVOR gratefuldy thanks captain Adrian Lane, relief master, and all the officers and crew for their cooperation and competancz. The research ... program WAFM COFE FiINGS is funded by the beean sciences Division of the National. Science Foundation. We al. 50 actnowledge support of the bio-optical and acoustic current profiling research by the oceanic frocesses branch of the National feronatics and Space faministration.

\section{INDEX TO TAELES AND FIGLIFES}

In what follows various data have been tabulated and four fjoures attached. The contents of these are as follows:

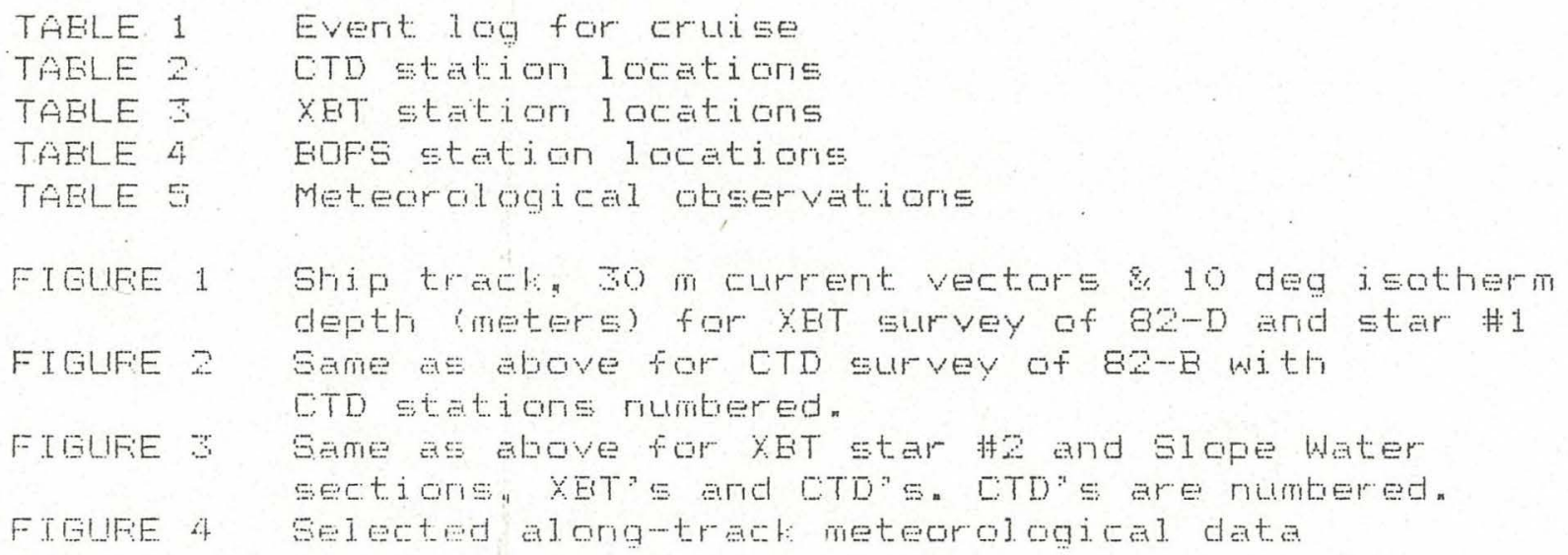




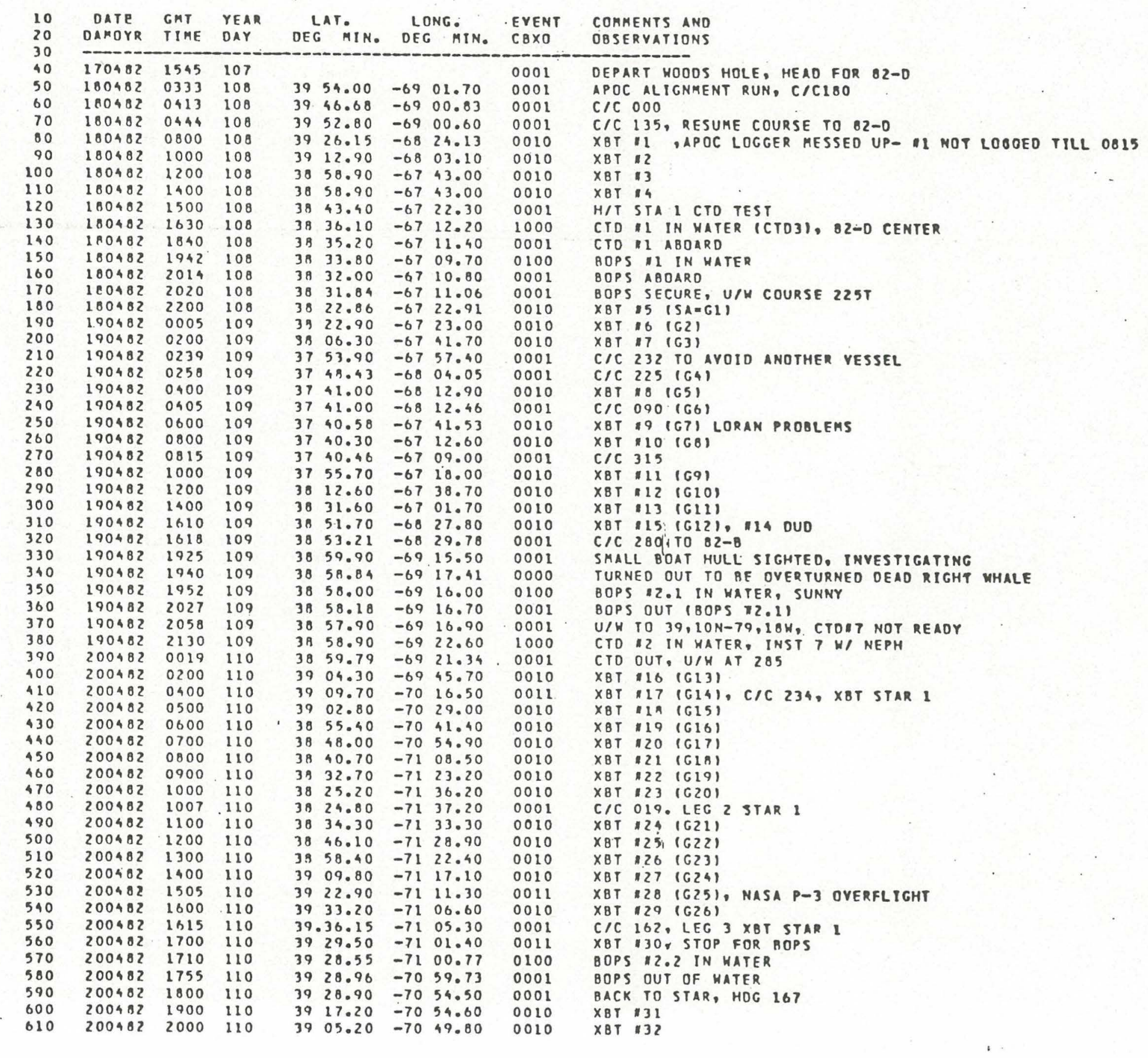




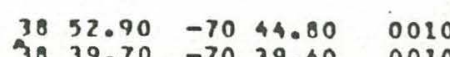
$\begin{array}{lllll}38 & 39.70 & -70 & 39.40 & 001\end{array}$ $\begin{array}{llllll}38 & 27.10 & -70 & 34.30 & 0010\end{array}$ $\begin{array}{llllllll}38 & 26.11 & -70 & 33.97 & 000\end{array}$ $\begin{array}{lllll}38 & 30.90 & -70 & 43.80 & 001\end{array}$ $\begin{array}{llllll}38 & 37.80 & -70 & 55.90 & 0010\end{array}$ $\begin{array}{lllll}38 & 44.80 & -71 & 08.20 & 0010\end{array}$ $\begin{array}{llll}38 & 52.40 & -7121.40 & 0010\end{array}$

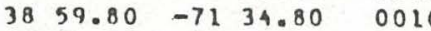
$\begin{array}{lllll}39 & 06.90 & -71 & 47.10 \quad 0010\end{array}$ $39 \quad 10.60 \quad-7153.80000$ $3911.80 \quad-7149.00 \quad 0010$ $\begin{array}{lllll}39 & 11.70 & -71 & 32.80 & 0010\end{array}$ $3911.40 \quad-70 \quad 59.10 \quad 0010$ $\begin{array}{llll}39 & 11.50 & -70 & 43.40 \quad 0010\end{array}$ $\begin{array}{lllll}39 & 11.60 & -70 & 27.90 & 0010\end{array}$ $\begin{array}{lllll}39 & 11.58 & -70 & 25.67 & 000\end{array}$

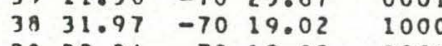
$\begin{array}{lllll}38 & 32.24 & -70 & 19.03 & 0001\end{array}$ $\begin{array}{lllll}39 & 32.23 & -70 & 19.03 & 0100\end{array}$ $\begin{array}{llllll}38 & 32.18 & -70 & 19.13 & 000\end{array}$ $\begin{array}{lllll}38 & 32.49 & -70 & 19.55 & 0001\end{array}$ $\begin{array}{lllll}38 & 36.80 & -70 & 28.60 & 0001\end{array}$ $\begin{array}{lllll}38 & 36.94 & -70 & 29.54 & 0100\end{array}$ $\begin{array}{lllll}38 & 36.56 & -70 & 29.59 & 0001\end{array}$ $\begin{array}{lllll}33 & 36.40 & -70 & 29.50 & 1000\end{array}$

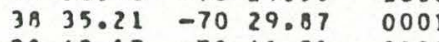
$\begin{array}{lllll}38 & 42.17 & -70 & 41.21 & 0100\end{array}$

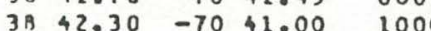
$\begin{array}{llll}38 & 38.30 & -70 & 41.90\end{array}$ उ月 $47.10 \quad-7051.65 \quad 0100$ $3846.25 \quad-70 \quad 51.70 \quad 0001$ 3月 $45.90 \quad-70 \quad 50.70 \quad 1000$ $\begin{array}{lllll}38 & 40.70 & -70 & 50.10 & 0001\end{array}$ $\begin{array}{lllll}38 & 52.20 & -71 & 02.10 & 0010\end{array}$ $\begin{array}{llllll}38 & 51.26 & -71 & 02.24 & 0100\end{array}$ उ8 $50.60-7102.40 \quad 0001$ $\begin{array}{lllll}38 & 50.36 & -71 & 02.47 & 0001\end{array}$ $\begin{array}{lllll}38 & 49.50 & -71 & 02.70 & 1000\end{array}$ उ月 $46.00-7105.000001$

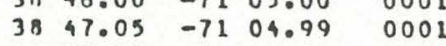

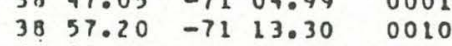

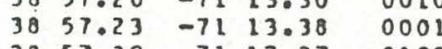
$3855.29-7113.37010$ $3855.15-7110.15$. 0000 $3854.69-7116.73000$ $3854.60-7117.75000$ $3854.20-7117.70 \quad 1000$ $3853.93-71 \quad 17.900000$ $3852.30=7118.10000$ $3852.09-7110.10 \quad 000$ 3859.090718 .34000 $3059.42-7120.700100$ $3059.37-7120.42 \quad 000$ $50.57-7120.40 \quad 1000$ $3850.60-7126.50 \quad 0001$ $3959.24 \quad-7128.18 \quad 0100$ $3905.10-71 \quad 39.50 \quad 1000$ $x \otimes T: 33$

X8T 134

$X B T: 35$

C/C $30 G T$

XBT 136

$X B T: 37$

$X B T \$ 38(635)$

$X 81: 3916361$

$\begin{array}{lll}X 8 T & 140 \quad(637)\end{array}$

XBT 141 (C38), 40 ON THE BATHY SYSTENS C/C 090

XBT 143 (G40), NOTES EST. RING CENTER POS.

XBT 144 (G1) $38,57.6 \mathrm{~K} 71.13 .2 \mathrm{~W}$

$X B T 145(62)$

$X 8 T: 46 \quad(G 3)$

$X 8 T \cap 47 \quad(64)$

$\begin{array}{llll}C & 172 \\ C & 172 & 1\end{array}$

CTO 3 TIN MATER

CTOPS OF IN WATER

BOPS OUT

U/H

BOPS IA IN NATER

$B O P S$ OUT

CTD I4 IN WATER

CTO OUT, U/W

BOPS 5 IN HATER

BOPS DUT

CTD 25 : IN YATER

CTO ABD, U/W

BOPS 16 IN WATER

BOPS DUT

CTD IO IN HATER

CTO OUT, U/K

XBT:48

BOPS 7 IN WATER

CTO 7 IN HATER

CTO ON ROARD

XIW $\triangle 49$

HIT STA O, RING CENTERP

BOPS $O$ IN KATER

U/M FOR ORIFTER DEPLOYMENT

DRIFTEIR LAUNCHEO HI SEDIMENT TRAPS

I $B$ IN MATER

LORAN C DATA CASSETTE TO STDE 2

CTO ON DECK, BRIOGE LOC

U/H, BRIDGE LOG

BOPS IN IN MATER

BOPS OUT

CTD 9 IN MATER

CTO DUT, U/W

BOPS 10 IN WATER

BOps out 
(Cont.)

$\begin{array}{llll}1230 & 230482 & 0055 & 113 \\ 1240 & 230482 & 0130 & 113 \\ 1250 & 230482 & 0218 & 113 \\ 1280 & 230482 & 0245 & 113 \\ 1270 & 230482 & 0252 & 113 \\ 1280 & 230482 & 0433 & 113 \\ 1290 & 230482 & 0505 & 113 \\ 1300 & 230482 & 0810 & 113 \\ 1310 & 230482 & 0823 & 113 \\ 1320 & 230482 & 0846 & 113 \\ 1330 & 230482 & 0557 & 113 \\ 1340 & 230482 & 0815 & 113 \\ 1350 & 230482 & 0838 & 113 \\ 1360 & 230482 & 1003 & 113 \\ 1370 & 230482 & 1043 & 113 \\ 1380 & 230482 & 1045 & 113 \\ 1390 & 230482 & 1100 & 113 \\ 1400 & 230482 & 1500 & 113 \\ 1410 & 230482 & 1534 & 113 \\ 1420 & 230482 & 1534 & 113 \\ 1430 & 230482 & 1640 & 113 \\ 1440 & 230482 & 1700 & 113 \\ 1450 & 230482 & 1750 & 113 \\ 1400 & 230482 & 1803 & 113 \\ 1470 & 230482 & 1825 & 113 \\ 1480 & 230482 & 1330 & 113 \\ 1490 & 230482 & 2015 & 113 \\ 1500 & 230482 & 2043 & 113 \\ 1510 & 230482 & 2140 & 113 \\ 1520 & 230482 & 2205 & 113 \\ 1530 & 230482 & 2210 & 113 \\ 1540 & 240482 & 0000 & 114 \\ 1550 & 240482 & 0021 & 114 \\ 1580 & 240492 & 0100 & 114 \\ 1570 & 240482 & 0113 & 114 \\ 1580 & 240482 & 0137 & 114 \\ 1590 & 240482 & 0138 & 114 \\ 1500 & 240482 & 0409 & 114 \\ 1610 & 240482 & 0446 & 114 \\ 1620 & 240482 & 0600 & 114 \\ 1630 & 240482 & 0507 & 114 \\ 1640 & 240482 & 0640 & 114 \\ 1550 & 240482 & 0641 & 114 \\ 1650 & 240482 & 1115 & 114 \\ 1670 & 240482 & 1212 & 114 \\ 1390 & 240482 & 1155 & 114 \\ 1690 & 240482 & 1340 & 114 \\ 1700 & 240482 & 1411 & 114 \\ 17100 & 240482 & 1523 & 114 \\ 1720 & 240482 & 1607 & 114 \\ 1730 & 240482 & 1717 & 114 \\ 1740 & 240482 & 1730 & 114 \\ 1750 & 240482 & 1820 & 114 \\ 1760 & 240482 & 2145 & 114 \\ 1770 & 240482 & 2149 & 114 \\ 1780 & 240482 & 2220 & 114 \\ 1790 & 240482 & 2330 & 114 \\ 1800 & 250482 & 0005 & 115 \\ 1810 & 250482 & 0010 & 115 \\ 1820 & 250482 & 0153 & 115 \\ 1830 & 250482 & 0316 & 115\end{array}$

$\begin{array}{llll}39 & 05.50 & -71 & 39.20\end{array}$

$\begin{array}{llll}39 & 06.94 & -71 & 42.79\end{array}$

$\begin{array}{llll}39 & 10.20 & -71 & 51.80\end{array}$

$\begin{array}{llll}39 & 10.20 & -71 & 51.30\end{array}$

$\begin{array}{llll}39 & 10.20 & -71 & 51.30\end{array}$

$3910.20-7149.80$

$\begin{array}{llll}39 & 15.50 & -72 & 03.20\end{array}$

$\begin{array}{llll}39 & 15.48 & -72 & 03.34\end{array}$

$\begin{array}{lllll}30 & 15.41 & -72 & 03.40\end{array}$

$\begin{array}{lllll}39 & 15.34 & -72 & 03.37\end{array}$

$39 \quad 15.40 \quad-72 \quad 03.10$

$\begin{array}{llll}39 & 15.40 & -72 & 03.00\end{array}$

$\begin{array}{llll}39 & 21.00 & -72 & 15.00\end{array}$

$\begin{array}{llll}39 & 21.94 & -72 & 15.94\end{array}$

$\begin{array}{llll}39 & 22.16 & -72 & 15.96\end{array}$

$\begin{array}{llll}39 & 22.60 & -72 & 16.20\end{array}$

$3946.12 \quad-7123.98$

$3946.00 \quad-71 \quad 23.40$

$3946.00 \quad-7123.60$

$3945.40 \quad-71 \quad 22.10$

$3945.20 \quad-7121.50$

$\begin{array}{llll}39 & 35.98 & -71 & 24.09\end{array}$

$3036.83-7123.80$

$\begin{array}{llll}39 & 36.80 & -71 & 22.50\end{array}$

$3936.00-71 \quad 18.50$

$3935.60 \quad-71 \quad 17.29$

$\begin{array}{llll}39 & 27.22 & -71 & 23.90\end{array}$

$\begin{array}{llll}39 & 27.00 & -71 & 22.50\end{array}$

$3927.00 \quad-7122.50$

$3925.20-7120.80$

$\begin{array}{llll}39 & 25.44 & -71 & 20.57\end{array}$

$\begin{array}{llll}39 & 19.40 & -71 & 23.30\end{array}$

$\begin{array}{llll}39 & 17.88 & -71 & 23.98\end{array}$

$\begin{array}{llll}39 & 17.79 & -71 & 22.55\end{array}$

$3917.80-7123.00$

$3917.30-71 \quad 15.90$

$3915.93-7116.21$

$3908.11-7124.03$

$\begin{array}{llll}39 & 08.10 & -71 & 23.01\end{array}$

$\begin{array}{llll}39 & 07.80 & -71 & 22.30\end{array}$

$\begin{array}{lllll}39 & 07.80 & -71 & 22.30\end{array}$

$\begin{array}{llll}39 & 04.80 & -71 & 17.50\end{array}$

$\begin{array}{lllll}33 & 57.70 & -71 & 24.30\end{array}$

$3900.41 \quad-71 \quad 21.25$

$3856.40-7126.00$

$\begin{array}{llll}38 & 56.04 & -71 & 26.82\end{array}$

$\begin{array}{llll}33 & 56.67 & -71 & 26.94\end{array}$

$\begin{array}{llll}38 & 56.16 & -71 & 25.64\end{array}$

$3947.60 \quad-7123.94$

$3845.92-7125.02$

$\begin{array}{llll}38 & 43.50 & -71 & 30.00\end{array}$

$\begin{array}{llll}3 \text { 月 } & 43.50 & -71 & 30.90\end{array}$

$\begin{array}{llll}38 & 43.16 & -71 & 31.29\end{array}$

$3938.50-7124.50$

$\begin{array}{llll}38 & 37.80 & -71 & 25.40\end{array}$

$\begin{array}{llll}38 & 37.80 & -71 & 25.40\end{array}$

39 $38.48 \quad-71 \quad 27.24$

$3838.10 .-7128.70$
CTD ARD, LOST XMISSION FROM DRIFTER

IN WATER

BOPS OUT

CTO "II IN HATER

CTD OUT

H NEXT STATION

il in Kater

BOPS OUT

CTO 12 IN HATEQ

CTO DUT

BOPS "IJ IN KATER

BOPS OUT

CTO "13 IN HATER

CTO OUT, U/H

BCPS 14 IN HATER

$B O P S$ OUT

CTO ON IN HATER

U/m

BOPS 15 IN HATER

BOPS ABOARD

CTO :IS IN HATER

CTO OUT

U/K

BOPS "I6 IN HATER

BOPS OUT

CTO II IN HATER

CTD ON DECK

$\mathrm{U} / \mathrm{H}$

BOPS $\$ 17$ IN HATER

BOPS OUT

CTD $N$ LT IN HATER

$U / H$

BOPS RIB IN HATER

BOPS OUT

CTO *IA IN HATER, HIRE LEVEL HINO PROBLEAS

CTO OUT, UTh

CTIFTER HEARD

ORTFTER HEARD, $71,45.738,40.9$ HOG NNE

CTD ABD. FISH PRSLMS, BOPS CABLE JUMPED SHIEVE

CTD 220 IN HATER, AFT HINCH

COM H B. HAHN, CAN CUT, CANNOT SHITCH REELS

CTOPS WT SHITCH CARLE TO BOPS

BOPS 50 ?

BOPS $Z 21$ IN HATER

BOPS OUT?

CTO "2I IN HATER

LEVEL WINO PRBLMS AT $1951 \mathrm{~m}$, FIXEO AT $0223 \mathrm{GmT}$ 


$\begin{array}{llll}1840 & 250482 & 0350 & 115 \\ 1850 & 250482 & 0445 & 115 \\ 1800 & 250482 & 0701 & 115 \\ 1970 & 250482 & 0711 & 115 \\ 1830 & 250582 & 0745 & 115 \\ 1890 & 250482 & 0828 & 115 \\ 1900 & 250482 & 0831 & 115 \\ 1910 & 250482 & 0900 & 115 \\ 1920 & 250482 & 0904 & 115 \\ 1930 & 250482 & 1115 & 115 \\ 1940 & 250482 & 1217 & 115 \\ 1950 & 250482 & 1415 & 115 \\ 1900 & 250482 & 1428 & 115 \\ 1970 & 250482 & 1457 & 115 \\ 1980 & 250482 & 1813 & 115 \\ 1990 & 250482 & 1930 & 115 \\ 2000 & 250482 & 1955 & 115 \\ 2010 & 250482 & 2000 & 115 \\ 2020 & 250432 & 2228 & 115 \\ 2030 & 250482 & 2230 & 115 \\ 2040 & 250482 & 2317 & 115 \\ 2050 & 260482 & 0005 & 116 \\ 2080 & 260482 & 0325 & 116 \\ 2070 & 260482 & 0341 & 116 \\ 2080 & 260482 & 0412 & 116 \\ 2090 & 260482 & 0426 & 116 \\ 2100 & 260482 & 0515 & 116 \\ 2110 & 260482 & 0524 & 116 \\ 2120 & 260482 & 0630 & 116 \\ 2130 & 260482 & 0635 & 116 \\ 2140 & 260492 & 0444 & 116 \\ 2150 & 260482 & 0651 & 116 \\ 2160 & 260482 & 0900 & 116 \\ 2170 & 260482 & 0915 & 116 \\ 2180 & 260482 & 1010 & 116 \\ 2190 & 260482 & 1227 & 116 \\ 2200 & 260482 & 1235 & 116 \\ 22100 & 260482 & 1305 & 116 \\ 2220 & 250482 & 1412 & 116 \\ 2230 & 260482 & 1640 & 116 \\ 2240 & 260482 & 1645 & 116 \\ 2250 & 260482 & 1716 & 116 \\ 2250 & 260482 & 1812 & 116 \\ 2270 & 260482 & 1817 & 116 \\ 2280 & 260482 & 1940 & 116 \\ 2290 & 260482 & 2052 & 116 \\ 2300 & 270497 & 0000 & 117 \\ 2310 & 270492 & 0220 & 117 \\ 2320 & 270482 & 0228 & 117 \\ 2330 & 270482 & 0255 & 117 \\ 2340 & 270482 & 0302 & 117 \\ 2350 & 270482 & 0535 & 117 \\ 2360 & 270482 & 0512 & 117 \\ 2370 & 270482 & 0710 & 117 \\ 2380 & 270482 & 0729 & 117 \\ 2390 & 270482 & 1034 & 117 \\ 2400 & 270488 & 1055 & 117 \\ 2410 & 270482 & 1150 & 117 \\ 2420 & 270482 & 1308 & 117 \\ 2430 & 270482 & 1325 & 117 \\ 2440 & 270482 & 1330 & 117 \\ & & & \end{array}$

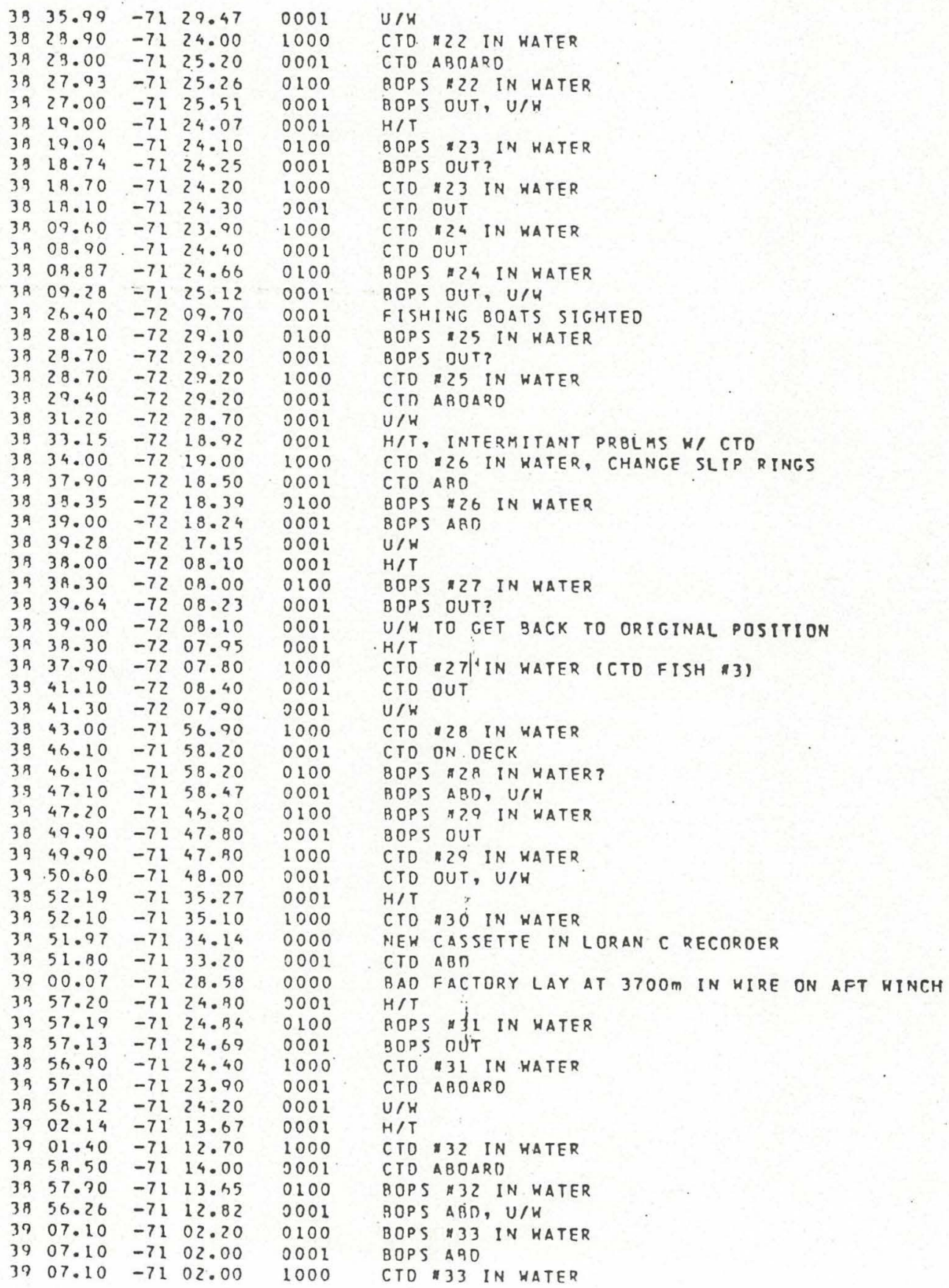

CTD 33 IN HATER 


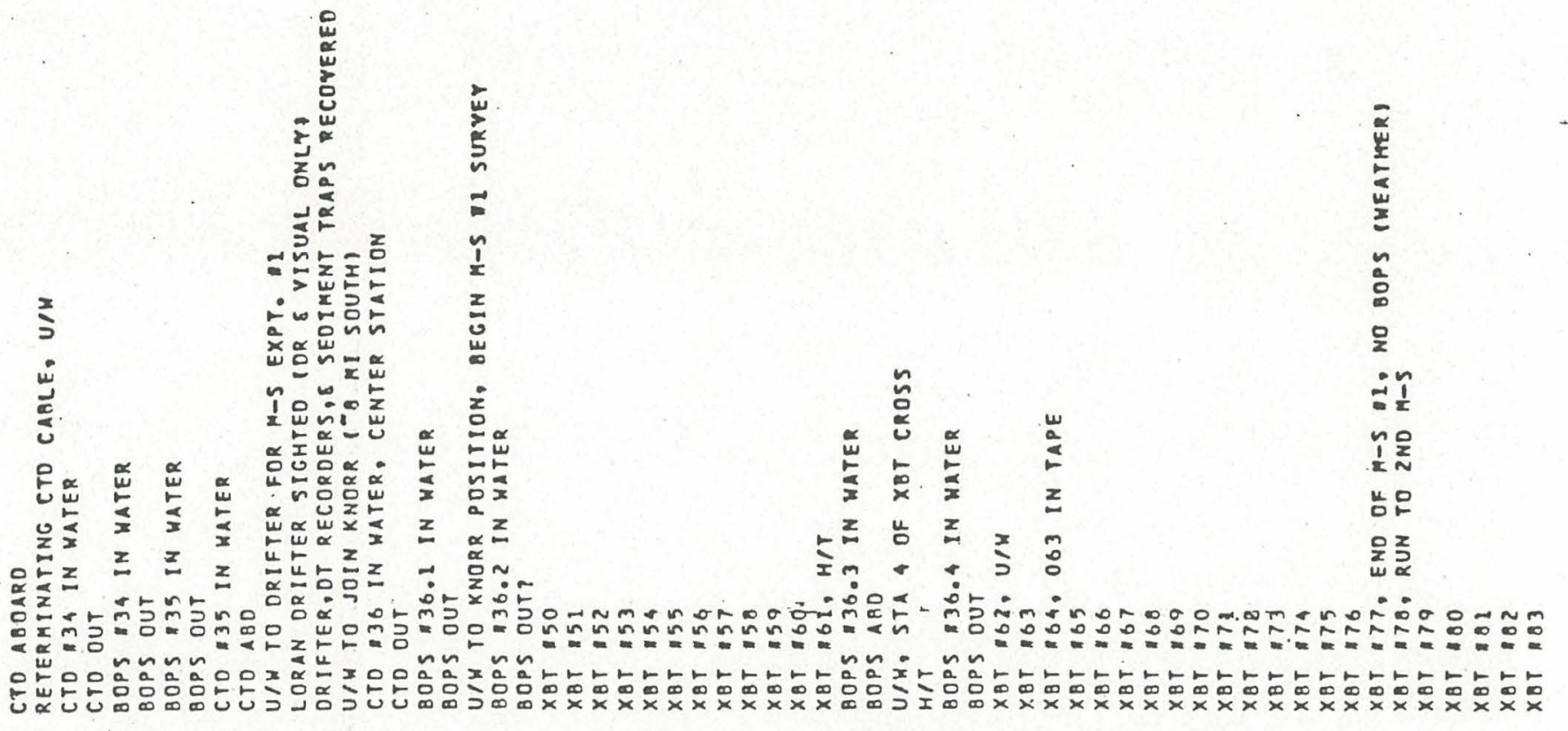

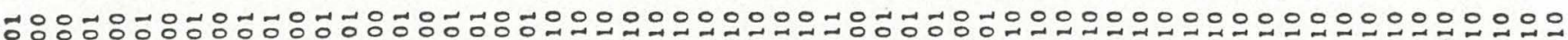

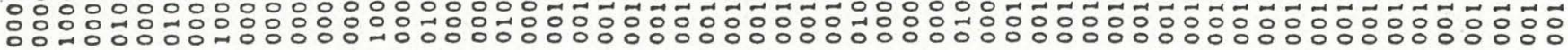

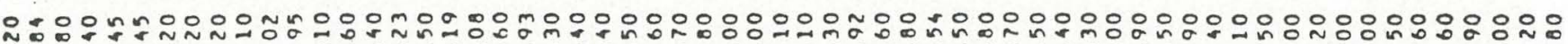

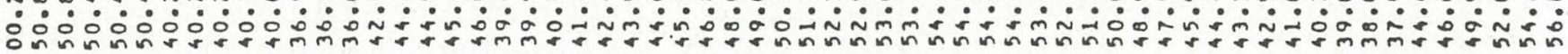

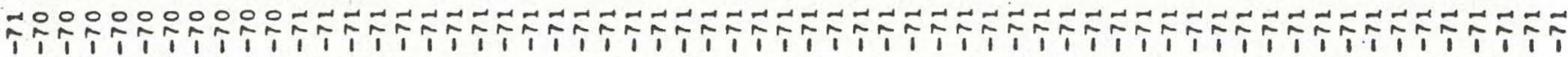

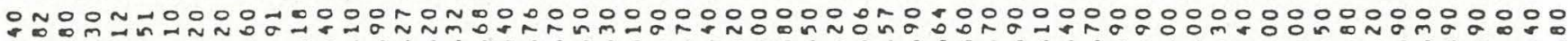

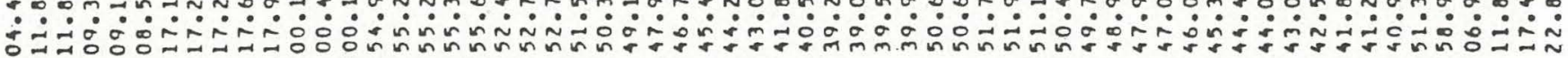

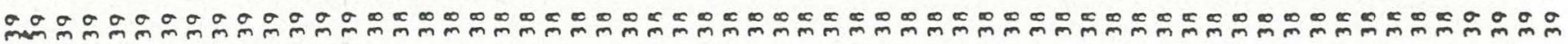

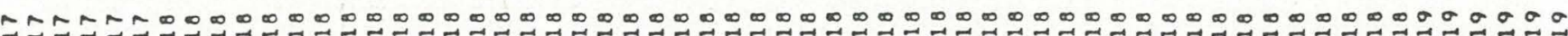

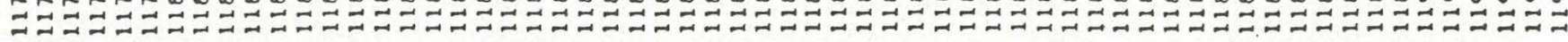

은운

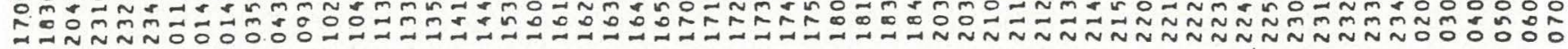

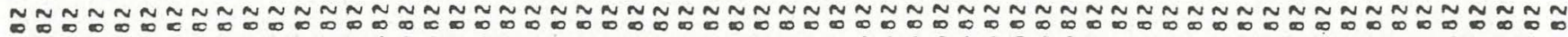

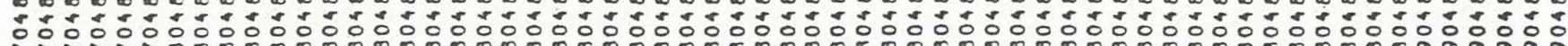

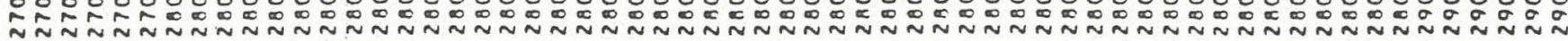

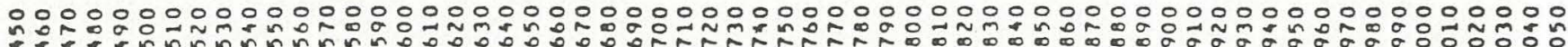

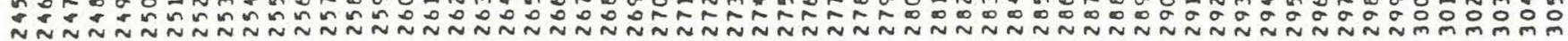




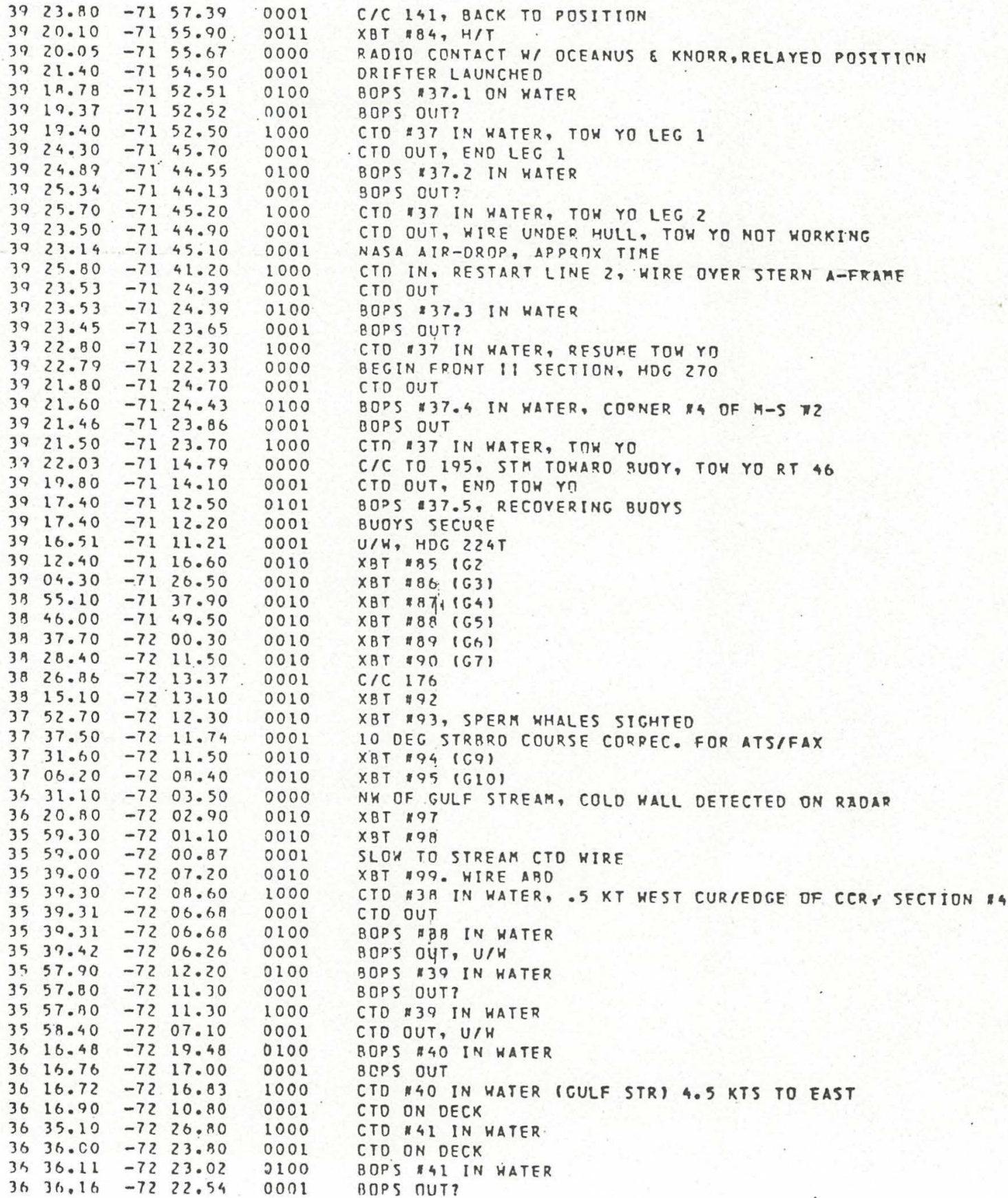

$\begin{array}{lllll}37 & 23.80 & -71 & 57.39 & 0001\end{array}$ $3920.10 \quad-7155.90: 001$ $7021.40-7154.50 \quad 000$ $39 \quad 18.78-7152.510100$ $3919.37-7152.520001$ $3919.40-7152.50$. 1000 $3924.30-7145.70 \quad 1000$ $3924.00-71.45 .55000$ $3925.34-7144.550100$ $3925.70-7145.20000$ $3925.70 \quad-7145.20 \quad 1000$ $39.50-7144.90 \quad 000$ $0254-7145.10 \quad 0001$ $3925.80-7141.20 \quad 1000$ $3923.53-7124.39000$ $\begin{array}{lllll}39 & 23.45 & -71 & 24.39 & 0100 \\ 39 & 22.45 & -71 & 23.65 & 0001\end{array}$ $\begin{array}{lllll}37 & 22.80 & -71 & 22.30 & 1000\end{array}$

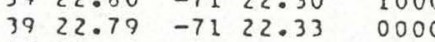
$3921.80 \quad-7124.70 \quad 0001$ $3921.60-7124.43 \quad 0100$ $3921.46 \quad-7123.86 \quad 000$ $3921.50 \quad-71 \quad 23.70 \quad 1000$ $3722.03 \quad-7114.790000$ $\begin{array}{lllll}39 & 22.03 & -71 & 14.79 & 0000\end{array}$ $3917.40-7112.50 \quad 0101$ $39 \quad 17.40 \quad-71 \quad 12.20 \quad 000$ $\begin{array}{lllll}39 & 16.51 & -71 & 11.21 & 0001\end{array}$ $\begin{array}{lllll}39 & 12.40 & -71 & 16.60 & 0010\end{array}$

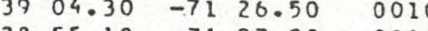
35 $55.10 \quad-71 \quad 37.90 \quad 0010$ $\begin{array}{lllll}38 & 46.00 & -71 & 49.50 & 0010\end{array}$

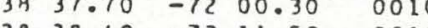
$\begin{array}{llll}34 & 28.40 & -72 & 11.50 \text {. } 0010\end{array}$ $\begin{array}{lllll}38 & 26.86 & -72 & 13.37 & 000\end{array}$ $3515.10-7213.100010$ $\begin{array}{llllll}37 & 52.70 & -72 & 12.30 & 0010\end{array}$ $3737.50-7211.74000$ $37.060-7211.50$ - 0010 $\begin{array}{lllll}37 & 06.20 & -72 & 0.40 & 0010\end{array}$ उम $31.10 \quad-72 \quad 03.50 \quad 0000$

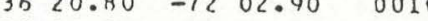
$\begin{array}{lllll}35 & 59.30 & -72 & 01.10 & 0010\end{array}$ $\begin{array}{lllll}35 & 57.00 & -72 & 00.87 & 000\end{array}$ $\begin{array}{lllll}35 & 39.00 & -72 & 07.20 & 0010\end{array}$ $35 \quad 39.30 \quad-7208.60 \quad 1000$ $\begin{array}{lllll}35 & 39.31 & -72 & 06.68 & 0001\end{array}$ $\begin{array}{lllll}35 & 30.31 & -72 & 06.68 & 0100\end{array}$ $\begin{array}{lllll}35 & 37.42 & -72 & 06.26 & 000\end{array}$ $\begin{array}{lllll}35 & 57.90 & -72 & 12.20 & 0100\end{array}$ $\begin{array}{lllll}35 & 57.80 & -72 & 11.30 & 000\end{array}$ $\begin{array}{lllll}35 & 57.80 & -72 & 11.30 & 1000\end{array}$

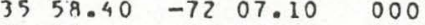
$\begin{array}{lllll}36 & 16.48 & -72 & 19.48 & 0100\end{array}$ $\begin{array}{lllll}36 & 16.76 & -72 & 17.00 & 0001\end{array}$ $\begin{array}{lllll}36 & 16.72 & -72 & 16.83 & 1000\end{array}$ $\begin{array}{lllll}36 & 16.90 & -72 & 10.80 & 0001\end{array}$ $\begin{array}{lllll}36 & 35.10 & -72 & 26.80 & 1000\end{array}$ $\begin{array}{lllll}36 & 36.00 & -72 & 23.80 & 0001\end{array}$ $\begin{array}{lllll}34 & 36.11 & -72 & 23.02 & 2100\end{array}$ $\begin{array}{lllll}36 & 36.16 & -72 & 22.54 & 0001\end{array}$

C/C 141, BACK TO POSITION

XBT $84, H / T$

RADIO CONTACT W/ OCEANUS E KNORR,RELAYED POSTTICN

BOPS $\$ 37.1$ ON HATER

BOPS OUT?

CTD \#37 IN HATER, TOH YO LEG 1

CTO OUT, END LEG 1

BOPS $\times 37.2$ IN HATER

SOPS OUT?

CTI 37 IN HATER, TOH YO LEG 2

CTO OUT, HIRE UNDER HULL, TOH YO NOT HORKINE

NASA ATR-ORDP, APPRTX TIME

CTO IN, RESTART LINE 2, HIRE OYER STERN A-FRAME CTO OUT

BOPS 237.3 IN HATER

$80 P S$ OUT?

CTO "37 IN HATER, RESUME TOH YD

BEGIN FRONT II SECTION, HOG 270

CTO OUT

BOPS $\$ 37.4$ IN YATER, COPNER $\$ 4$ OF M-S $\$ 2$

BOPS OUT

CTI 137 IN HATER, TOK YO

C/C TO 195. STA TOHARO SUOY, TOH YO RT 46

CTO OUT, END TOH YM

BOPS 37.5 , RECOVERING BUOYS

BUOYS SECURE

$U / H, H D G 224$

XBT $135 \quad 162$

$X 8 T: 86(63)$

$X B T=87_{1}(64)$

$X B T$ \# $8 \&$ ( $(65)$

$X B T \# 89(G)$

$X B T=90(G 7)$

$\mathrm{C} / \mathrm{C} 176$

$X B T \# 92$

XBT M93, SPERM HHALES SIGHTED

10 DEG STRBRD COURSE CORPEC. FOR ATSIFAX

$X B T \| 94(C 9)$

XBT 195 (G10

NK OF GULF STREAM, COLO haLl DETECTED ON RADAR

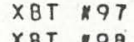

SLOH TO STREAK CTD HIRE

XBT "99. HIRE ABO

CTO H3R IN HATER, . 5 KT WEST CUR/EDGE OF CCR \& SECTION 4 CTD OUT

BOPS $B B$ IN WATER

BOPS OUT, U/H

BOPS "3O IN HATER

BOPS OUT?

CTO 39 IN HATER

CTO OUT, U/H

BOPS 40 IN HATER

BCPS OUT

CTD "4O IN HATER (CULF STR) 4.5 KTS TO EAST

CTO ON DECK

CTO $/ 41$ IN HATER

BOPS 41 IN HATER 


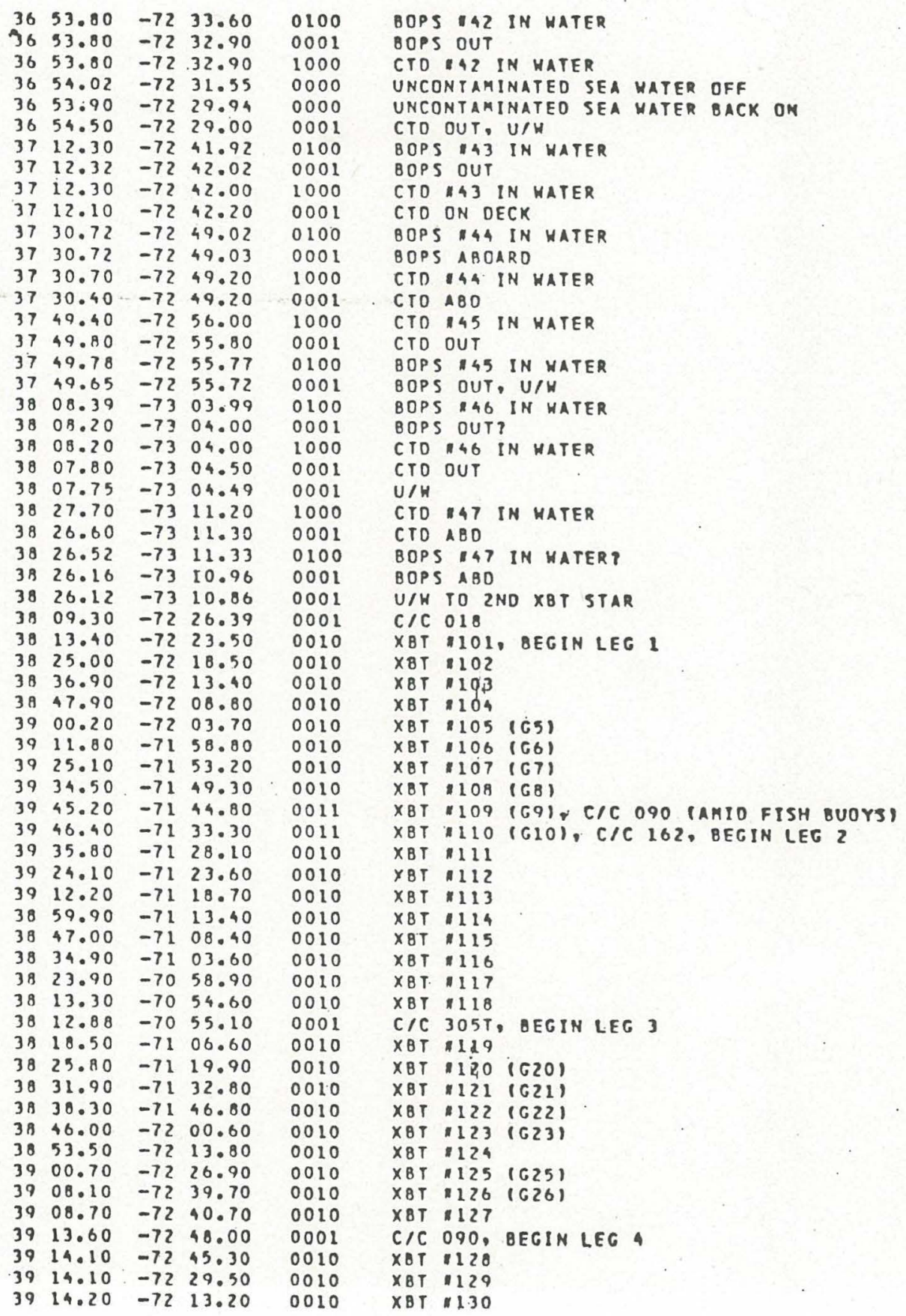




$\begin{array}{llll}4280 & 050582 & 0000 & 125 \\ 4290 & 050582 & 0010 & 125 \\ 4300 & 050582 & 0100 & 125 \\ 4310 & 050582 & 0200 & 125 \\ 4320 & 050582 & 0231 & 125 \\ 4330 & 050582 & 0300 & 125 \\ 4340 & 050582 & 0306 & 125 \\ 4350 & 050582 & 0500 & 125 \\ 4360 & 050582 & 0600 & 125 \\ 4370 & 050582 & 0700 & 125 \\ 4380 & 050582 & 0745 & 125 \\ 4390 & 050582 & 0800 & 125 \\ 4400 & 050582 & 0900 & 125 \\ 4410 & 050582 & 1000 & 125 \\ 4420 & 050582 & 1100 & 125 \\ 4430 & 050582 & 1120 & 125 \\ 4440 & 050582 & 1200 & 125 \\ 4450 & 050582 & 1300 & 125 \\ 4460 & 050582 & 1400 & 125 \\ 4470 & 050582 & 1500 & 125 \\ 4480 & 050582 & 1600 & 125 \\ 4490 & 050582 & 1700 & 125 \\ 4500 & 050582 & 1800 & 125 \\ 4510 & 050582 & 1900 & 125 \\ 4520 & 050582 & 2000 & 125 \\ 4530 & 050582 & 2016 & 125 \\ 4540 & 050582 & 2100 & 125 \\ 4550 & 050582 & 2200 & 125 \\ 4560 & 050582 & 2300 & 125 \\ 4570 & 060582 & 0000 & 126 \\ 4580 & 060582 & 0100 & 126 \\ 4590 & 060582 & 0200 & 126 \\ 4600 & 060582 & 0300 & 126 \\ 4610 & 060582 & 0358 & 126 \\ 4620 & 060582 & 0402 & 126 \\ 4630 & 060582 & 0500 & 126 \\ 4640 & 060582 & 0600 & 126 \\ 4650 & 060582 & 0700 & 126 \\ 4660 & 060582 & 0800 & 126 \\ 4670 & 060582 & 0900 & 126 \\ 4680 & 060582 & 0917 & 126 \\ 4690 & 060582 & 1416 & 126 \\ 4700 & 060582 & 1429 & 126 \\ 4710 & 060582 & 1459 & 126 \\ 4720 & 060582 & 1600 & 126 \\ 4730 & 060582 & 1700 & 126 \\ 4740 & 060582 & 1715 & 126 \\ 4750 & 060582 & 1724 & 126 \\ 4760 & 060582 & 1745 & 126 \\ 4770 & 060582 & 1750 & 126 \\ 4780 & 060582 & 2124 & 126 \\ 4790 & 060582 & 2130 & 126 \\ 4800 & 060582 & 2300 & 126 \\ 4810 & 070582 & 0000 & 127 \\ 4820 & 070582 & 0020 & 127 \\ 4830 & 070582 & 0045 & 127 \\ 4840 & 070582 & 0054 & 127 \\ 4850 & 070582 & 0457 & 127 \\ 4860 & 070582 & 0510 & 127 \\ 4870 & 070582 & 0540 & 127 \\ 4880 & 070582 & 0600 & 127 \\ & & & \end{array}$

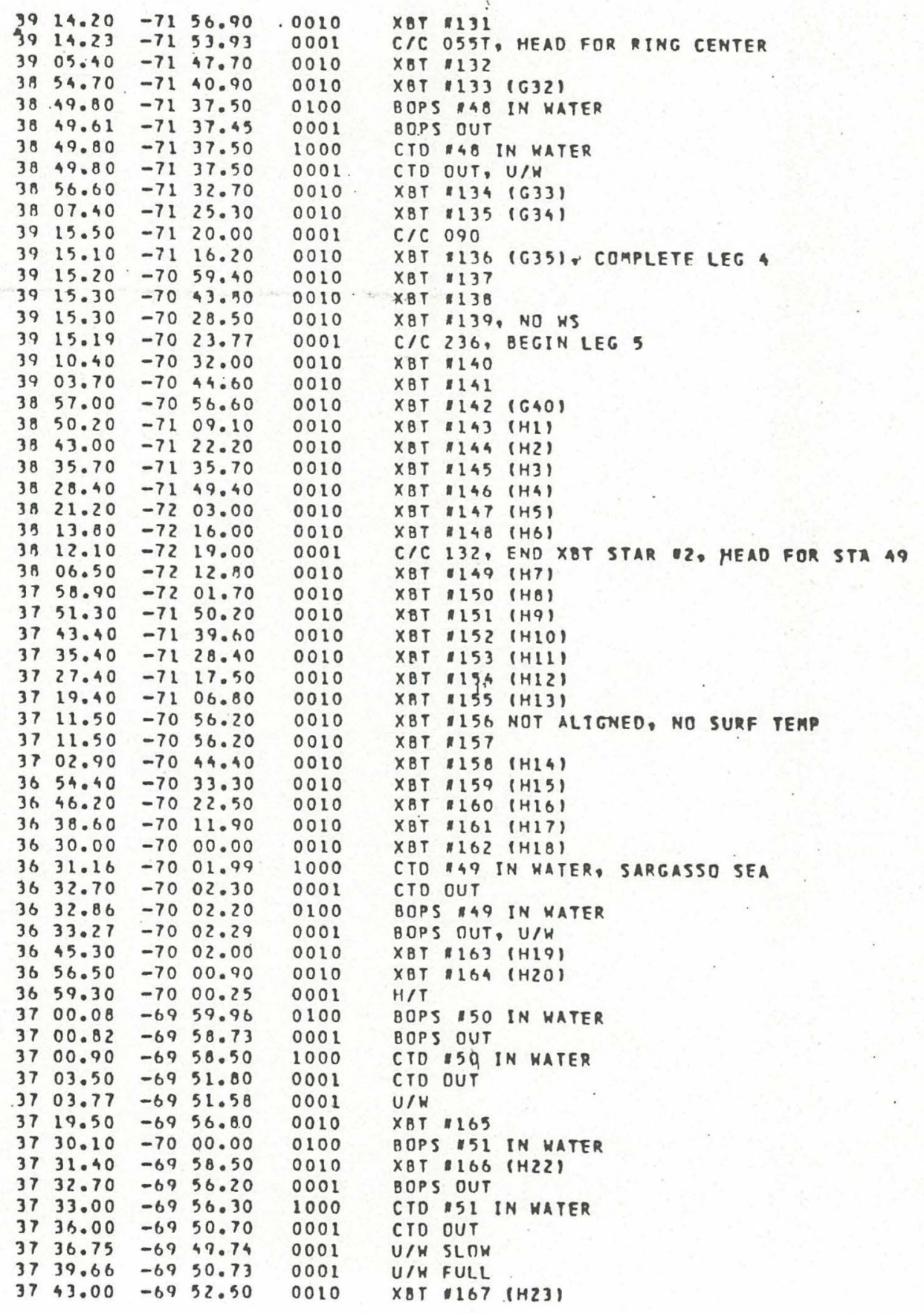


TABLE 1: EN83 EVENT LOG

(Cont.)

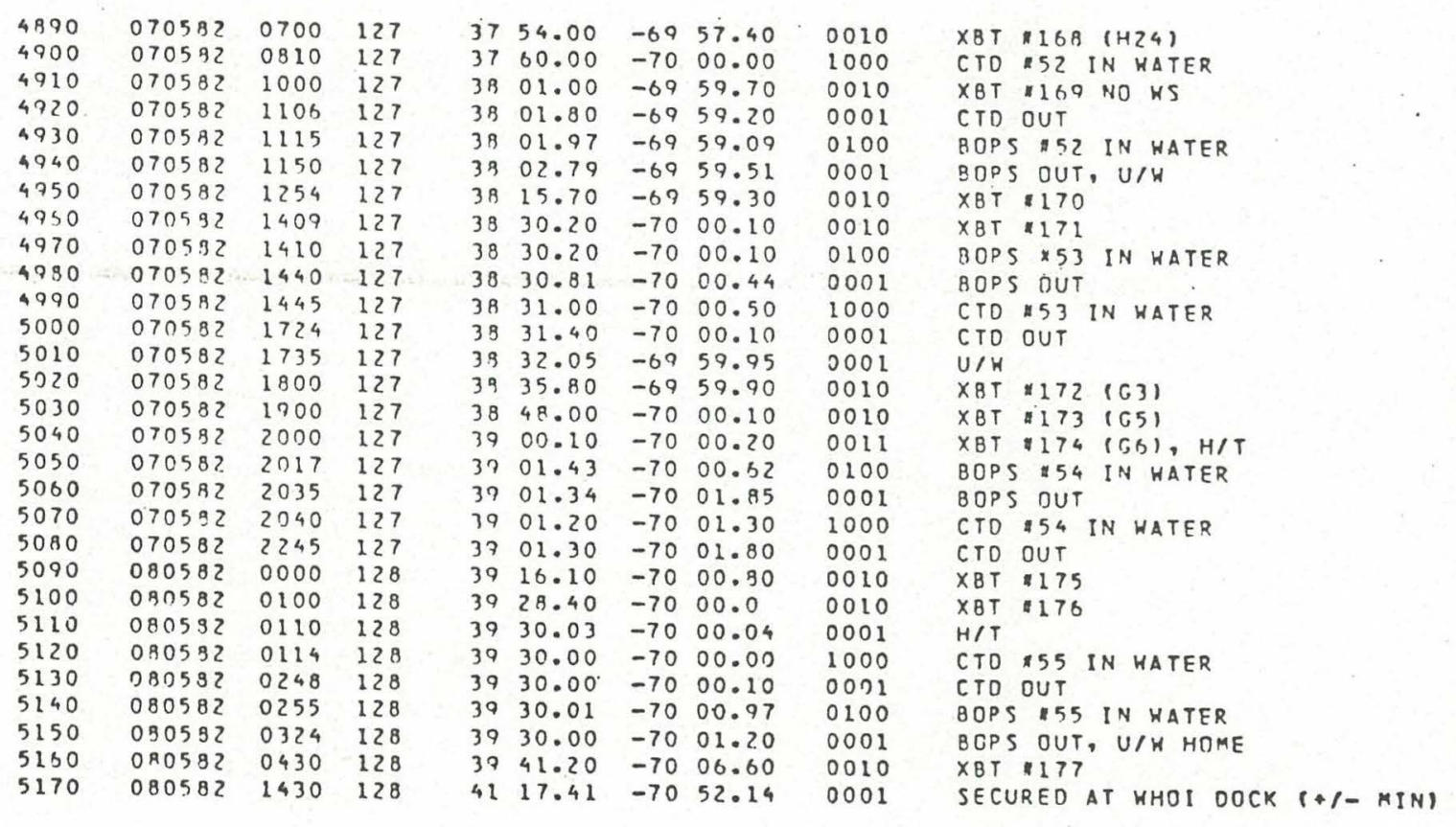


-TABLE 2: CTD STATION LOCATIONS

\begin{tabular}{|c|c|c|c|c|c|c|c|c|c|}
\hline \multicolumn{2}{|c|}{ DATE } & \multirow{2}{*}{$\begin{array}{l}C M T \\
\text { YIMF }\end{array}$} & \multirow{2}{*}{$\begin{array}{c}\text { JUL } I A N \\
D A Y\end{array}$} & \multicolumn{2}{|c|}{ LATITUDE } & \multicolumn{2}{|c|}{ LONGITUDF } & \multicolumn{2}{|c|}{ EVENT } \\
\hline DAY & MnS. & & & $D E G$. & - MIN. & DEG. & - MIN. & & \\
\hline 18 & 4 & 1630 & 109 & 38 & 36.10 & -67 & 12.20 & CTD & $\# 1$ \\
\hline 19 & 4 & 2130 & 107 & 38 & 58.90 & -59 & 22.50 & CTD & $4: 2$ \\
\hline 21 & 4 & 1446 & 111 & 38 & 31.97 & -70 & 19.02 & $\operatorname{cro}$ & 43 \\
\hline 21 & 4 & 1936 & 111 & 38 & 36.40 & -70 & $? 9.50$ & CTD & 144 \\
\hline 71 & 4 & 2317 & 111 & 38 & $42 \cdot 30$ & -70 & 41.00 & CTO & $\sharp 5$ \\
\hline 27 & 4 & 350 & $11 ?$ & 38 & 45.90 & -70 & 50.70 & CTO & \pm 6 \\
\hline $2 ?$ & 4 & 940 & 112 & 38 & 49.50 & -71 & 2.70 & CTO & 47 \\
\hline 22 & 4 & 1537 & 112 & 38 & 54.20 & -71 & 17.70 & Cro & 48 \\
\hline 22 & 4 & 1924 & 112 & 38 & 59.37 & -71 & 29.40 & CTD & 49 \\
\hline 22 & 4 & 2304 & 112 & 39 & 5.10 & -71 & 39.50 & CTO & $\# 10$ \\
\hline 23 & 4 & 252 & 113 & 39 & 10.20 & -71 & 51.30 & CTD & 411 \\
\hline 23 & 4 & 657 & 113 & 39 & 15.34 & -72 & 3.37 & $\operatorname{cto}$ & $\forall 12$ \\
\hline 23 & 4 & 1045 & 113 & 39 & 22.16 & -72 & 15.96 & CTR & $\# 13$ \\
\hline 23 & 4 & 1534 & 113 & 39 & 46.00 & -71 & 23.60 & CTD & \pm 14 \\
\hline 23 & 4 & 1830 & 113 & 39 & 36.80 & -71 & 22.50 & CTO & $\leftleftarrows 15$ \\
\hline 23 & 4 & $2 ? 10$ & 113 & 39 & 27.00 & -71 & 22.50 & CTO & $\$ 16$ \\
\hline 24 & 4 & 138 & 114 & 39 & 17.80 & -71 & 23.00 & CTD & $* 17$ \\
\hline 24 & 4 & 641 & 114 & 39 & 7.80 & -71 & 22.30 & CTD & $\# 18$ \\
\hline 24 & 4 & 1212 & 114 & 38 & 57.70 & -71 & 24.30 & CTD & $\$ 19$ \\
\hline 2.4 & 4 & 1730 & 114 & 38 & 47.60 & -71 & 24.00 & CTO & \pm 20 \\
\hline 25 & 4 & 10 & 115 & 38 & 37.80 & -71 & 25.40 & CTO & ب. 21 \\
\hline 25 & 4 & 445 & 115 & 38 & 28.90 & -71 & 24.00 & CTO & $\# 22$ \\
\hline 25 & 4 & 904 & 125 & 38 & 18.70 & -71 & 24.20 & Cro & 923 \\
\hline 25 & 4 & 1217 & 115 & 38 & 9.60 & -71 & 23.90 & CTD & \pm 24 \\
\hline 25 & 4 & 2000 & 115 & 38 & 28.70 & -72 & 29.20 & CTO & 42.5 \\
\hline 26 & 4 & 5 & 115 & 38 & 34.00 & -72 & 19.00 & CTD & $\$ 26$ \\
\hline 26 & 4 & 651 & 115 & 38 & 37.90 & -72 & 7.80 & Cro & 427 \\
\hline 26 & 4 & 1010 & 115 & 38 & 43.00 & -71 & 56.90 & CTD & $\$ 28$ \\
\hline 26 & 4 & 16,45 & 116 & 38 & 49.90 & -71 & 47.90 & CTn & $\approx 29$ \\
\hline 26 & 4 & 1817 & 115 & 38 & 52.10 & -71 & 35.10 & CTD & $\$ 30$ \\
\hline 27 & 4 & 302 & 117 & 38 & 56.90 & -71 & 24.40 & CTO & $\$ 31$ \\
\hline 27 & 4 & 729 & 117 & 39 & 1.40 & -71 & 12.70 & CTD & $\$ 32$ \\
\hline 27 & 4 & 1330 & 117 & 39 & 7.10 & -71 & 2. 00 & CTR & $\# 33$ \\
\hline 27 & 4 & 2042 & 117 & 39 & 11.80 & -70 & 50.80 & CTD & $\forall 34$ \\
\hline 29 & 4 & 145 & 119 & 39 & 17.20 & -70 & 40.20 & CTD & 435 \\
\hline 28 & 4 & 1136 & 113 & 38 & 54.90 & -71 & 42.40 & CTO & $\approx 36$ \\
\hline 29 & 4 & 1352 & 117 & 39 & 19.40 & -71 & 52.50 & C.TO & $* 37$ \\
\hline 29 & 4 & 1738 & 119 & 39 & 25.70 & -71 & 45.20 & CTD & $\$ 37$ \\
\hline 29 & 4 & 1955 & 119 & 39 & 25.80 & -71 & 41.20 & CTO & $* 37$ \\
\hline 30 & 4 & 354 & 120 & 39 & 22.80 & -71 & 22.30 & CTO & $: 37$ \\
\hline 30 & 4 & 740 & $1 ? 0$ & 39 & 21.50 & -71 & 23.70 & CTD & $: 37$ \\
\hline 1 & 5 & 1309 & 121 & 35 & 39.30 & -72 & $8.4,0$ & CTD & $\$ 38$ \\
\hline 1 & 5 & 1900 & 121 & 35 & 57.80 & -72 & 11.30 & CTO & 439 \\
\hline$?$ & 5 & 47 & 122 & 36 & 16.72 & -72 & 15.83 & CTO & 140 \\
\hline$?$ & 5 & 630 & 122 & 36 & 35.10 & -72 & $? 5.80$ & CTD & \pm 41 \\
\hline 2 & 5 & 1252 & $12 ?$ & 36 & 53.80 & -72 & 32.90 & CTD & $\$ 42$ \\
\hline 2 & 5 & 2000 & $12 ?$ & 37 & 12.30 & -72 & 42.00 & CTD & 143 \\
\hline 3 & 5 & 31 & 123 & 37 & 30.70 & -72 & 47.20 & CTO & $\$ 44$ \\
\hline 3 & 5 & 417 & 123 & 37 & 49.40 & -72 & 56.00 & CTO & $\pi 45$ \\
\hline 3 & 5 & 858 & 1.? 3 & 38 & 8.20 & -73 & 4.00 & CTD & 440 \\
\hline 3 & 5 & 1230 & 123 & 38 & 27.70 & -73 & 11.20 & CTD & \pm 47 \\
\hline 5 & 5 & 300 & 125 & 38 & 49.80 & -71 & 37.50 & CTD & 148 \\
\hline$h$ & 5 & 927 & 125 & 36 & $31 \cdot 16$ & -70 & 1.99 & C.TO & 144 \\
\hline 6 & 5 & 1750 & 126 & 37 & 0.90 & $-6,4$ & 58.50 & CTO & $=50$ \\
\hline 7 & 5 & 54 & 127 & 37 & 33.00 & -69 & 56.30 & CTO & 451 \\
\hline 7 & 5 & 810 & 127 & 17 & $0: 0.00$ & -70 & 3.00 & CTO & $=5,2$ \\
\hline 7 & 5 & 1445 & $1 ? 7$ & 38 & 31.00 & -70 & 3.50 & (II) & 253 \\
\hline 7 & 5 & 2040 & 127 & 39 & 1.20 & -70 & 1.30 & CTO & 454 \\
\hline 8 & 5 & 114 & 128 & 37 & 30.00 & -70 & 0.00 & $\cos$ & $\because 55$ \\
\hline
\end{tabular}


TABLE 3: XBT STATION LOCATIONS

SHIP: ENDEAVTR

\begin{tabular}{|c|c|c|c|c|c|c|c|c|c|}
\hline \multicolumn{2}{|c|}{ DATE } & \multirow{2}{*}{$\begin{array}{l}C, M T \\
T I: 4 F\end{array}$} & \multirow{2}{*}{$\begin{array}{c}\text { JULIAN } \\
\text { DAY }\end{array}$} & \multicolumn{2}{|c|}{ LATITUDF } & \multicolumn{2}{|c|}{ LDNG ITUDF } & \multicolumn{2}{|c|}{ EVENT } \\
\hline DAY & MOS. & & & $D E G$. & MIN. & $D F G$ & - $M I N$. & & \\
\hline 18 & 4 & 800 & 108 & 34 & 26.15 & -68 & 24.13 & $\times B T$ & $\# 1$ \\
\hline 18 & 4 & 1000 & 108 & 39 & 12. 30 & -68 & 3.10 & $X R T$ & +2 \\
\hline 18. & 4 & 1200 & 108 & 38 & 58.80 & -67 & 43.00 & $\times B T$ & 13 \\
\hline 18 & 4 & 1400 & 108 & 38 & 58.90 & -67 & 43.00 & Xar & 44 \\
\hline 18 & 4 & 2200 & 108 & 38 & $? ? .86$ & -67 & 22.91 & $\times 9 r$ & $: 5$ \\
\hline 19 & 4 & 5 & 109 & 38 & 22.30 & -67 & 23.00 & $X \cap T$ & 46 \\
\hline 19 & 4 & 200 & 109 & 38 & 6.30 & -67 & 41.70 & $\times B T$ & $\# 7$ \\
\hline 19 & 4 & 400 & 100 & 37 & 41.00 & -68 & 12.90 & $\times 2 T$ & $\# 8$ \\
\hline 19 & 4 & 600 & 109 & 37 & 40.58 & -67 & 41.53 & $X P T$ & $\$ 9$ \\
\hline 19 & 4 & 400 & 109 & 37 & 40.30 & -67 & 12.50 & $\times 3 T$ & $* 10$ \\
\hline 19 & 4 & 1000 & 109 & 37 & 55.70 & -67 & 18.00 & $\times 3 r$ & $\$ 11$ \\
\hline 19 & 4 & 1200 & 109 & 39 & 12.60 & -67 & 38.70 & $X \cap T$ & $\forall 12$ \\
\hline 19 & 4 & 1400 & 109 & 38 & 31.50 & -67 & 1.70 & $X \unrhd T$ & 413 \\
\hline 19 & 4 & 1610 & 109 & 38 & 51.70 & -68 & 27.80 & XAT & 415 \\
\hline 20 & 4 & 200 & 110 & 39 & 4.30 & -69 & 45.70 & $\times B T$ & 416 \\
\hline 20 & 4 & 400 & 110 & 39 & 9.70 & -70 & 16.50 & $\times 9 T$ & $: 17$ \\
\hline 20 & 4 & 500 & 110 & 39 & 2.80 & -70 & 29.00 & $\times B T$ & 418 \\
\hline 20 & 4 & 600 & 110 & 38 & 55.40 & -70 & 41.40 & XBT & 419 \\
\hline 20 & 4 & 700 & 110 & 38 & 48.00 & -70 & 54.90 & $\times 3 T$ & 120 \\
\hline 20 & 4 & 800 & 110 & 38 & 40.70 & -71 & 8.50 & $\times 3 T$ & 421 \\
\hline 20 & 4 & 900. & 110 & 38 & 32.70 & -71 & 23.20 & $\times 3 \mathrm{~T}$ & $\$ 22$ \\
\hline 20 & 4 & 1000 & 110 & 39 & 25.20 & -71 & 36.20 & $X \cap T$ & $1: 23$ \\
\hline 20 & 4 & 1100 & 110 & 38 & 34.30 & -71 & 33.30 & $X R T$ & $\$ 2.4$ \\
\hline 20 & 4 & 1200 & 110 & 38 & 45.10 & -71 & 24.90 & $\times 3 T$ & 425 \\
\hline 20 & 4 & 1300 & 110 & 39 & 59.40 & -71 & $\frac{72}{2} .40$ & $\times 2 T$ & $\div 26$ \\
\hline 20 & 4 & 1400 & 110 & 39 & 9.20 & -71 & 17.10 & $\times 3 T$ & $* 27$ \\
\hline 20 & 4 & 1505 & 110 & 39 & $2 ? \cdot 90$ & -71 & 11.30 & $\times 8 T$ & :1: 28 \\
\hline 20 & 4 & 1500 & 110 & 37 & 33.20 & $-7 \cdot 1$ & b. 50 & $X: T$ & 1129 \\
\hline 20 & 4 & 1700 & 110 & 37 & 2.3 .50 & -71 & 1.40 & $X P . T$ & $\$ 30$ \\
\hline 20 & 4 & 1900 & 110 & 39 & $17 . ? 0$ & -70 & 54.50 & $\times 3 T$ & $\Rightarrow 31$ \\
\hline $20^{\circ}$ & 4 & 2000 & 110 & 39 & 5.20 & -70 & 49.80 & XAT & $\$ 32$ \\
\hline 20 & 4 & 2100 & 110 & 38 & $5 ? .90$ & -70 & 44.90 & $\times 9 T$ & 433 \\
\hline 20 & 4 & 2200 & 120 & 39 & 39.70 & -70 & 39.40 & $X \cap T$ & \pm 34 \\
\hline 20 & 4 & 2300 & 110 & 38 & 2.7 .10 & -70 & 34.30 & YBT & 435 \\
\hline 21 & 4 & 0 & 111 & 38 & 30.70 & -70 & 43.90 & $X 2 T$ & 136 \\
\hline 21 & 4 & 100 & 111 & 38 & 37.80 & -70 & 55.30 & $\times 3 T$ & 437 \\
\hline 21 & 4 & 200 & 111 & 38 & 44.80 & -71 & 8.20 & $\times B T$ & 438 \\
\hline 21 & 4 & 300 & 111 & 39 & 52.40 & -71 & 21.40 & $\times A T$ & 439 \\
\hline 2.1 & 4 & 400 & 111 & 33 & 57.80 & -71 & 34.80 & $\times B T$ & $\$ 40$ \\
\hline 21 & 4 & 500 & 111 & 39 & 5.90 & -71 & 47.10 & $\times B T$ & 441 \\
\hline 21 & 4 & 500 & 111 & 37 & 11.90 & -71 & 49.00 & $Y ? T$ & 1142 \\
\hline 21 & 4 & 700 & 111 & 39 & 11.70 & -71 & 32.80 & $Y Q T$ & 443 \\
\hline$? .1$ & 4 & 800 & 111 & 39 & 11.50 & -71 & 16.20 & $X R T$ & 444 \\
\hline 21 & 4 & 900 & 111 & 37 & 11.40 & -70 & 59.10 & $\times 3 T$ & $\$ 45$ \\
\hline 2.1 & 4 & 1000 & 111 & 39 & 11.50 & -70 & 4.3 .40 & $X B T$ & 446 \\
\hline 21 & 4 & 1100 & 111 & 37 & 11.50 & -70 & 27.79 & $X 2 T$ & 144 \\
\hline 22 & 4 & $84 ?$ & 112 & 33 & 52.20 & -71 & 2.20 & $X \approx T$ & 148 \\
\hline 22 & 4 & $134 h$ & 112 & 39 & 57.20 & -71 & 13.30 & $\times 3 T$ & 449 \\
\hline 23 & 4 & $1+10$ & 118 & 38 & 52.70 & -71 & 40.30 & $X B T$ & $\$ 50$ \\
\hline $2 a$ & 4 & $1+20$ & 118 & 39 & 51.50 & -71 & 41.40 & $X \cap T$ & 451 \\
\hline 29 & 4 & 16,30 & 118 & $3 R$ & 50.30 & -71 & 42.40 & $Y \cap T$ & 452 \\
\hline 28 & 4 & 16,40 & 113 & 39 & 40.10 & -71 & 43.50 & $\times 4 T$ & 453 \\
\hline 28 & 4 & 16,50 & $11 \%$ & $3 n$ & 47.77 & -71 & 44.60 & $\times 3 T$ & 454 \\
\hline 28 & 4 & 1700 & 118 & 39 & 40.70 & -71 & 45.70 & $x ? T$ & 455 \\
\hline 2.3 & 4 & 1710 & 118 & $3 x$ & 45.40 & -71 & $4 t=90$ & $\times: 3 T$ & 456 \\
\hline 29 & 4 & 1720 & 113 & 38 & 44.70 & -71 & 4.9 .00 & $X \cap T$ & $: 457$ \\
\hline
\end{tabular}


TABLE 3: XBT STATION LOCATIONS

(Cont.)

\begin{tabular}{|c|c|c|c|c|c|c|c|c|}
\hline 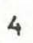 & $1+00$ & 124 & 38 & 38.30 & -71 & 46.80 & $X \Omega T$ & 1122 \\
\hline 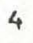 & 1700 & 124 & 38 & $4 h .00$ & -72 & 0.60 & $\times 3 T$ & 9123 \\
\hline 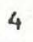 & 1400 & 124 & 38 & 53.50 & $-7 ?$ & 13.80 & $X B r$ & 4124 \\
\hline 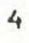 & 1900 & 12.4 & 39 & 0.70 & -72 & 26.90 & $Y A T$ & 1125 \\
\hline 6 & 2000 & 124 & 39 & 8.10 & -72 & $39.7 n$ & $X B T$ & 4126 \\
\hline 4 & 2003 & 124 & 39 & 8.70 & -72 & 40.70 & $X R T$ & $\$ 127$ \\
\hline 4 & 2100 & 124 & 39 & 14.10 & $-7 ?$ & 45.30 & $\times B T$ & $\# 128$ \\
\hline 4 & 2200 & 124 & 39 & 14.10 & -72 & 29.50 & $X B T$ & $\$ 129$ \\
\hline 4 & 2300 & 124 & 39 & 14.20 & -72 & 13.20 & $\mathrm{XOT}$ & 1130 \\
\hline 5 & 5 & 125 & 39 & 14.20 & -71 & $5 h .90$ & $x B T$ & 1131 \\
\hline 5 & 100 & 125 & 39 & 5.40 & -71 & 47.70 & $\times 3 \mathrm{~T}$ & 4132 \\
\hline 5 & 200 & 125 & 38 & 54.70 & -71 & 40.90 & $X \cap T$ & 4133 \\
\hline 5 & 600 & 125 & 38 & 56.60 & -71 & 32.70 & $x x^{a} T$ & $\because 134$ \\
\hline 5 & 700 & 125 & 39 & 7.40 & -71 & 25.30 & $\times B T$ & 4135 \\
\hline 5 & 300 & 125 & 39 & 15.10 & -71 & 16.20 & $\times 9 \mathrm{~T}$ & $\# 136$ \\
\hline 5 & 900 & 125 & 39 & 15.20 & -70 & 59.40 & $\times B T$ & $H 137$ \\
\hline 5 & 1000 & 125 & 39 & 15.30 & -70 & 43.80 & $\times 3 T$ & 4.28 \\
\hline 5 & 1100 & 125 & 39 & 15.30 & -70 & 29.50 & $X P T$ & 4139 \\
\hline 5 & 1200 & 125 & 39 & 10.40 & -70 & 32.00 & X8T & $=140$ \\
\hline 5 & 1300 & 125 & 39 & 3.70 & -70 & 44.50 & $\times 3 \mathrm{~T}$ & $\$ 141$ \\
\hline 5 & 1400 & 125 & 38 & 57.00 & -70 & 56.60 & $\times 3 T$ & $\$ 142$ \\
\hline 5 & 1500 & 125 & 33 & 50.20 & -71 & 9.10 & $\times 3 T$ & 1143 \\
\hline 5 & 1600 & 125 & 3.3 & 43.00 & -71 & 22.20 & $\times 3 T$ & "144 \\
\hline 5 & 1700 & 125 & 3.3 & 35.70 & -71 & 35.70 & $X B T$ & 1145 \\
\hline 5 & 1800 & 125 & 38 & 28.40 & -71 & 49.40 & $X 8 T$ & $\# 146$ \\
\hline 5 & 1900 & 125 & 38 & 21.20 & -72 & 3.00 & $X B T$ & 1147 \\
\hline 5 & 2000 & 125 & 39 & 13.80 & -72 & 16.00 & $\times 8 T$ & 1148 \\
\hline 5 & 2100 & 125 & 38 & 6.50 & -72 & 12.90 & $\times B Y$ & $* 149$ \\
\hline 5 & 2200 & 125 & 37 & 58.90 & -72 & 1.70 & $\times 3 T$ & *150 \\
\hline 5 & 2300 & 125 & 37 & 51.30 & -71 & 50.20 & $X R T$ & $\# 151$ \\
\hline 6 & $n$ & 126 & 37 & 43.40 & -71 & 39.50 & $X 9 T$ & $\forall 152$ \\
\hline 6 & 100 & 126 & 37 & 35.40 & -71 & 28.40 & $X \cap T$ & ${ }^{4153}$ \\
\hline 0 & 200 & 126 & 37 & 27.40 & -71 & 17.50 & $X \cong T$ & $\# 154$ \\
\hline 6 & 300 & 126 & 37 & 19.40 & -71 & 5.80 & $X R T$ & $\# 155$ \\
\hline 6 & 358 & 126 & 37 & 11.50 & -70 & $5 b .20$ & $X B T$ & $\because 156$ \\
\hline 6 & $40 ?$ & 126 & 37 & 11.50 & -70 & 56.20 & $\times 91$ & $\# 157$ \\
\hline 6 & 500 & 126 & 37 & 2.90 & -70 & 44.40 & $Y Q T$ & $\# 158$ \\
\hline 6 & 600 & 126 & 36 & 54.40 & -70 & 33.30 & $\times 8 T$ & $\# 159$ \\
\hline 5 & .700 & 126 & $3 h$ & 45.20 & -70 & 22.50 & $X \cap T$ & $\# 160$ \\
\hline 6 & 800 & 126 & 36 & 38.60 & -70 & 11.90 & $X R T$ & $\# 161$ \\
\hline 6 & 900 & 126 & 36 & 30.00 & -70 & 0.00 & $X \cap T$ & 4162 \\
\hline 6 & 1600. & 126 & 36 & 45.30 & -70 & 2.00 & $X P . T$ & 1163 \\
\hline 6 & 1700 & 126 & 35 & 56.50 & -70 & 0.90 & $\times 8 T$ & $\$ 164$ \\
\hline 6 & 2300 & 126 & 37 & 17.50 & -69 & 56.80 & $X P T$ & 9165 \\
\hline 7 & 20 & 127 & 37 & 31.40 & -69 & 58.50 & $\times B T$ & H166 \\
\hline 7 & 600 & 127 & 37 & 43.00 & -69 & 52.50 & $X \cap T$ & $\# 167$ \\
\hline 7 & 700 & 127 & 37 & 54.00 & -69 & 57.40 & $X R T$ & $\$ 168$ \\
\hline 7 & 1000 & 127 & 3я & 1.00 & -69 & 59.70 & $X \cap T$ & 4169 \\
\hline 7 & 1254 & 127 & 38 & 15.70 & -69 & 59.30 & $\times 3 T$ & $\$ 170$ \\
\hline 7 & $140 ?$ & 127 & 38 & 30.20 & -70 & 0.10 & $\times \cap T$ & $\# 171$ \\
\hline 7 & 1800 & 127 & 38 & 35.80 & -69 & 59.90 & $X 9 T$ & $* 172$ \\
\hline 7 & 1900 & 127 & 38 & 49.00 & -70 & 0.10 & $X ? T$ & $y 173$ \\
\hline 7 & 2000 & 127 & 37 & 0.10 & -70 & 0.20 & $X R T$ & 1174 \\
\hline 8 & 5. & 128 & 39 & 15.10 & -70 & 0.80 & $X \cap T$ & $\$ 175$ \\
\hline 3 & 100 & 128 & 37 & 29.40 & -70 & 0.00 & $X 8 T$ & $\forall 176$ \\
\hline 8 & 430 & 128 & & 41.20 & -70 & 5. 60 & $\times 3 T$ & 1177 \\
\hline
\end{tabular}


TABLE 4: BOPS STATION LOCATIONS

\begin{tabular}{|c|c|c|c|c|c|c|c|c|c|}
\hline \multicolumn{2}{|c|}{ DATE } & \multirow{2}{*}{$\begin{array}{l}\text { GMT } \\
\text { TIME }\end{array}$} & \multirow{2}{*}{$\begin{array}{c}\text { JUL I AN } \\
\text { DAY }\end{array}$} & \multicolumn{2}{|c|}{ LATI TUDE } & \multicolumn{2}{|c|}{ LONGITUDE } & \multicolumn{2}{|c|}{ EVENT } \\
\hline DAY & MOS. & & & DES. & - $k I N$ & DEG. & MIN. & & \\
\hline 18 & 4 & 1942 & 108 & 38 & 33.80 & -67 & 9.70 & B. $3 P 5$ & $\# 1$ \\
\hline 19 & 4 & 1952 & 109 & 38 & 58.00 & -69 & 16.00 & QJPPS & 72.1 \\
\hline 20 & 4 & 1710 & 110 & 37 & 2.8 .55 & -71 & 0.77 & PIIPS & 42.2 \\
\hline 21 & 4 & 1715 & 111 & $3 B$ & 32.23 & -70 & 19.03 & BJPS & * 3 \\
\hline 21 & 4 & 1853 & 111 & 38 & 36.94 & -70 & 29.54 & EIPS & $\# 4$ \\
\hline 21 & 4 & 2252 & 111 & 39 & 42.17 & -70 & 41.21 & BOPS & $\sharp 5$ \\
\hline 22 & 4 & 310 & 112 & $3 \pi$ & 47.10 & -70 & 51.65 & RDPS & 46 \\
\hline 22 & 4 & 856 & $11 ?$ & 33 & 51.26 & -71 & 2.24 & BOPS & 47 \\
\hline 22 & 4 & 1403 & 112 & 38 & 57.29 & -71 & 13.37 & BOPS & \# 8 \\
\hline 22 & 4 & 1853 & 122 & 38 & 59.50 & -71 & 28.70 & BJPS & 49 \\
\hline 22 & 4 & 2243 & 112 & 38 & 59.24 & -71 & 28.18 & BDPS & $* 10$ \\
\hline 23 & 4 & 218 & 113 & 39 & 10.70 & -71 & 51.80 & SOPS & HI 1 \\
\hline 23 & 4 & 623 & 113 & 39 & 15.48 & -72 & 3.34 & QJPS & $\# 12$ \\
\hline 23 & 4 & 1003 & 113 & 39 & 21.00 & -72 & 15.00 & ADPS & 413 \\
\hline 23 & 4 & 1500 & 113 & 39 & 46.12 & -71 & 23.98 & AOPS & $\$ 14$ \\
\hline 23 & 4 & 1803 & 113 & 39 & 36.87 & -71 & $2 \cdot 3.30$ & QOPS & 415 \\
\hline 23 & 4 & 2140 & 113 & 37 & 27.22 & -71 & 23.90 & DJPS & \pm 16 \\
\hline 24 & 4 & 113 & 114 & 39 & 17.83 & -71 & 23.78 & DOPS & 417 \\
\hline 24 & 4 & 607 & 114 & 39 & R. 10 & -71 & 23.61 & ADPS & $\# 18$ \\
\hline 24 & 4 & 1523 & 114 & 38 & 56.67 & -71 & 26.94 & $30 P 5$ & $\$ 19$ \\
\hline 24 & 4 & 2149 & 114 & 38 & 43.50 & -71 & 30.90 & $37 P 5$ & $\because 20$ \\
\hline 24 & 4 & 2330 & 114 & 38 & 38.50 & -71 & 24.50 & RJPS & $\# 21$ \\
\hline 25 & 4 & 711 & 115 & 38 & 2.7 .93 & -71 & 25.26 & QDPS & 422 \\
\hline 25 & 4 & 831 & 115 & 38 & 17.04 & -71 & 24.10 & RIPS & $\$ 23$ \\
\hline 25 & 4 & 1429 & 115 & 38 & R. 87 & -71 & 24.66 & QOPS & \# 24 \\
\hline 25 & 4 & 1930 & 115 & 38 & 28.10 & -72 & 29.10 & BOPS & 425 \\
\hline $2 h$ & 4 & 341 & 116 & 38 & 38.35 & $-72=$ & $18.39^{\circ}$ & BOPS & $\# 26$ \\
\hline 25 & 4 & 524 & 116 & 39 & 38.30 & -72 & 8.00 & RTPS & 427 \\
\hline 26 & 4 & 1235 & 116 & 38 & 46.10 & -71 & 58.20 & R.JPS & $\div 28$ \\
\hline 26 & 4 & 1412 & 116 & 39 & 47.20 & -71 & 46.20 & QJPS & 429 \\
\hline 27 & 4 & 228 & 117 & 38 & 57.19 & -71 & 24.84 & ROPS & $\$ 31$ \\
\hline 27 & 4 & 1055 & 117 & 39 & 57.90 & -71 & 13.65 & $33 P 5$ & $\$ 32$ \\
\hline 27 & 4 & $130 R$ & 117 & 39 & 7.10 & -71 & 2.20 & RDPS & $: 33$ \\
\hline 27 & 4 & $232 ?$ & 117 & 37 & 9.12 & -70 & 50.45 & 30PS & \#34 \\
\hline 28 & 4 & 110 & 119 & 39 & 17.10 & -70 & 40.20 & POPS & $\# 35$ \\
\hline 28 & 4 & 1350 & 118 & 38 & $55 . ? 0$ & -71 & 44.50 & $B \cap P S$ & $\# 35.1$ \\
\hline 28 & 4 & 1530 & 118 & 38 & 52.40 & -71 & 39.50 & RJPS & $\$ 36.2$ \\
\hline 28 & 4 & 1811 & 118 & 38 & 39.06 & -71 & 52.92 & RJPS & $\# 36.3$ \\
\hline 28 & 4. & 2035 & 118 & 38 & 50.60 & -71 & 54.50 & 3005 & $\pm 3 h .4$ \\
\hline 29 & 4 & 1311 & 119 & 39 & 19.78 & -71 & 52.51 & S.JPS & $\# 37$ \\
\hline 29 & 4 & 1648 & 119 & 39 & 24.99 & -71 & 44.55 & RDPS & 437.2 \\
\hline 30 & 4 & 241 & 120 & 39 & 23.53 & -71 & 24.30 & aOf & $\# 37.3$ \\
\hline 30 & 4 & 707 & 120 & 37 & 21.60 & -71 & 24.43 & ADPS & \pm 37.4 \\
\hline 30 & 4 & 1230 & 120 & 39 & 17.40 & -71 & 12.50 & $3.3 P 5$ & \pm 37.5 \\
\hline 1 & 5 & 1511 & 121 & 35 & 39.31 & -72 & G.SB & ROPS & $\div 38$ \\
\hline 1 & 5 & 1820 & 121 & 35 & 57.90 & -72 & 12.20 & AOPS & 1439 \\
\hline 2 & 5 & 14 & $12 ?$ & 36 & 16.48 & -72 & 19.49 & DOPS & $\pi 40$ \\
\hline 2 & 5 & 936 & 127 & 36 & $3 f .11$ & -72 & 23.02 & R.JPS & $\$ 41$ \\
\hline 2 & 5 & 1217 & 122 & $3 t$ & 53.80 & -72 & 33.60 & BOPS & $\pi 4 ?$. \\
\hline 2 & 5 & 1930 & 122 & 37 & 12.30 & -72 & 41.92 & RTPS & $\$ 43$ \\
\hline 2 & 5 & 2357 & 122 & 37 & $30.7 ?$ & -72 & 49.02 & R.JPS & $: 44$ \\
\hline 3 & 5 & 610 & 123 & 37 & 49.79 & -72 & 55.77 & nחPS & $\because 45$ \\
\hline 3 & 5 & 829 & 123 & 38 & 8.39 & -73 & 3.99 & QYPS & 140. \\
\hline 3 & 5 & 1415 & 123 & 38 & $? 6.5 ?$ & -73 & 11.33 & RTPS & 1447 \\
\hline 5 & 5 & 231 & 125 & 34 & 47.80 & -71 & 37.50 & a.sP & $\$ 48$ \\
\hline 6 & 5 & 1429 & 126 & 36 & 32.86 & -70 & 2.20 & ROPS & $=49$ \\
\hline 6 & 5 & 172.4 & 126 & 37 & $0 . \cap ?$ & -69 & 59.04 & PIPS & $=0$ \\
\hline 7 & 5 & $\therefore 0$ & 127 & 37 & 30.10 & -70 & 0.00 & "ะา & $\because 71$ \\
\hline 7 & 5 & 1115 & 127 & 33 & 1.77 & $-(2)$ & 50.07 & RIPS & 452 \\
\hline 7 & 5 & 1410 & 177 & 3.9 & 30.20 & -70 & 0.10 & ח.TPS & 453 \\
\hline 7 & 5 & 2017 & 127 & 30 & 1.43 & -70 & $0.6 . ?$ & :าคร & in $; 4$ \\
\hline 8 & 5 & 254 & 128 & 31) & 30.01 & -70 & 0.97 & DIPS & is \\
\hline
\end{tabular}


TABLE 3: XBT STATION LOCATIONS

(Cont.)

\begin{tabular}{|c|c|c|c|c|c|c|c|c|c|}
\hline 28 & 4 & 1730 & 118 & 39 & 43.00 & -71 & 49.00 & $X \cap T$. & 458 \\
\hline 28 & 4 & 1740 & 118 & $3 r$ & 41.80 & -71 & 50.10 & $\times B T$ & * 59 \\
\hline 2.8 & 4 & 1750 & 11.8 & 38 & 40.50 & -72 & 51.10 & XPT & $=00$ \\
\hline 28 & 4 & 1800 & 118 & 38 & 39.20 & -71 & 52.30 & $\times 3 T$ & 461 \\
\hline 28 & 4 & 2110 & 118 & $3 R$ & 51.90 & -71 & 54.70 & $\times 3 T$ & 452 \\
\hline 28 & 4 & 2120 & 118 & 38 & 51.10 & -71 & 53.50 & $\times 9 T$ & 463 \\
\hline 28 & 4 & 2130 & 118 & 38 & 50.40 & -71 & $5 ? .40$ & XBT & $\mathrm{H}_{4} 4$ \\
\hline 28 & 4 & 2140 & 118 & 38 & 49.70 & -71 & 51.30 & $X B T$ & 465 \\
\hline 23 & 4 & 2150 & 118 & 38 & 48.90 & -71 & 50.00 & $X B T$ & 466 \\
\hline 28 & 4 & 2200 & 118 & 38 & 47.90 & -71 & 48.70 & $X \cap T$ & HST \\
\hline 28 & 4 & 2210 & 118 & 38 & 47.00 & -71 & 47.50 & $\times 9 T^{\circ}$ & 468 \\
\hline 28 & 4 & 2220 & 118 & 38 & 45.00 & -71 & 45.90 & $\times 9 T$ & 1169 \\
\hline 28. & 4. & 22.30 & 118 & 38 & 45.30 & -71 & 44.40 & $X R T$ & 170 \\
\hline 28 & 4 & 2240 & 118 & 38 & 44.40 & -71 & 43.10 & $\times 3 r$ & $\# 71$ \\
\hline 29 & 4 & 2250 & 118 & 38 & 44.00 & -71 & 42.50 & XBT & 472 \\
\hline 28 & 4 & 2300 & 118 & 38 & 43.00 & -71 & 41.00 & $X Q Y$ & 473 \\
\hline 28 & 4. & 2310 & 118 & 38 & 42.50 & -71 & 40.20 & $X Q T$ & 174 \\
\hline 28 & 4 & 2320 & 118 & 38 & 41.90 & -71 & 39.00 & $X B T$ & $\# 75$ \\
\hline 28 & 4 & 2330 & 118 & 38 & 41.20 & -71 & 38.00 & $\times B T$ & 476 \\
\hline 28 & 4 & 2340 & 118 & 38 & 40.90 & -71 & 37.50 & XBT & $\approx 77$ \\
\hline 29 & 4 & 200 & 119 & 33 & 51.30 & -71 & 44.60 & $\times B T$ & 478 \\
\hline 29 & 4 & 300 & 119 & 39 & 5.8 .90 & -71 & 46.60 & $\times 3 T$ & 179 \\
\hline 29 & 4 & 400 & 119 & 39 & 6.90 & -71 & 49.90 & Xe.T & 480 \\
\hline 29 & 4 & 500 & 119 & 39 & 11.90 & -71 & 52.00 & $X B Y$ & 481 \\
\hline 29 & 4 & 600 & 119 & 39 & 17.40 & -71 & 54.20 & $X B T$ & 482 \\
\hline 29 & 4 & 700 & 119 & 37 & 22.80 & -71 & 56.90 & $\times 35$ & 1183 \\
\hline 29 & 4 & 750 & 119 & 39 & 20.10 & -71 & 55.90 & $X \cap T$ & $\# 84$ \\
\hline 30 & 4 & 1402 & 120 & 39 & 12.40 & -71 & 16.60 & XRT & $\# 9.5$ \\
\hline 30 & 4 & 1500 & 120 & 39 & 4.30 & -71 & 25.50 & $\times 9 T$ & 486 \\
\hline 30 & 4 & 1600 & 120 & 39 & 55.10 & -71 & 37.90 & $\times B T$ & 187 \\
\hline 30 & 4 & 1700 & 120 & 38 & 46.00 & -71 & 49.50 & $X R T$ & 489 \\
\hline 30 & 4 & 1800 & 120 & 38 & 37.70 & $-7 ?$ & 0.30 & $\times 9 T$ & 489 \\
\hline 30 & 4 & 1900 & 120 & 39 & 78.40 & -72 & 11.50 & $\times \cap T$ & 490 \\
\hline 30 & 4 & 2010 & 120 & 38 & 15.10 & -72 & 13.10 & $X R T$ & 492 \\
\hline 30 & 4 & 2200 & 120 & 37 & 52.70 & -72 & 12.30 & $\times B T$ & 493 \\
\hline 1 & 4 & 0 & 121 & 37 & 31.50 & -72 & 11.50 & $X R T$ & $=994$ \\
\hline 1 & 5 & 200 & 121 & 37 & 6.20 & -72 & 8.40 & $\times B T$ & $\not 95$ \\
\hline 1 & 5 & 500 & 121 & 36 & 20.80 & -72 & 2.90 & $\times B T^{\circ}$ & 497 \\
\hline 1 & 5 & 800 & 121 & 35 & 57.30 & -72 & 1.10 & $\times B T$ & 438 \\
\hline 1 & 5 & 1230 & 121 & 35 & 39.00 & -72 & 7.20 & $\times 3 T$ & 499 \\
\hline 3 & 5 & 1900 & 123 & 38 & 13.40 & -72 & 23.50 & X?T & 4101 \\
\hline 3 & 5 & 2000 & 123 & .39 & 25.00 & -72 & 18.50 & $\times P T$ & $* 102$ \\
\hline 3 & 5 & 2100 & 123 & 39 & 36.70 & -72 & 13.40 & $\times 3 T$ & 4103 \\
\hline 3 & 5 & 2200 & 123 & 38 & 47.90 & -72 & 8.90 & $\times 3 T$ & $\$ 104$ \\
\hline 3 & 5 & 2302 & 123 & 37 & 0.20 & -72 & 3.70 & $\times 7 T$ & $\# 105$ \\
\hline 4 & 5 & 0 & 124 & 39 & 11.90 & .71 & 58.90 & $X \cap T$ & $\$ 106$ \\
\hline 4 & 5 & 105 & 124 & 39 & 23.10 & -71 & 53.20 & $\times 9 T$ & 4107 \\
\hline 4 & 5 & 159 & 124 & 39 & 34.50 & $-7 I$ & 43.30 & $\times 9 T$ & 4108 \\
\hline 4 & 5 & 300 & 124 & 39 & 45.20 & -71 & 44.80 & $X Q T$ & 4109 \\
\hline 4 & 5 & 400 & 124 & 39 & 46.40 & -71 & 33.30 & $X ? T$ & $\$ 110$ \\
\hline 4. & 5 & 500 & 124 & 39 & 35.80 & -71 & 28.10 & $X R T$ & $\$ 111$ \\
\hline 4 & 5 & 600 & 124 & 39 & 24.10 & -71 & 23.60 & $\times B T$ & $\because 112$ \\
\hline 4 & 5 & $70 n$ & 12.4 & 37 & 12.20 & -71 & 18.70 & $X B T$ & 4113 \\
\hline 4 & 5 & 900 & 124 & 38 & 53.30 & -71 & 13.40 & $X P T$ & $\$ 114$ \\
\hline 4 & 5 & 900 & 124 & 33 & 47.00 & -71 & 3.40 & $x \cap T$ & 4115 \\
\hline 4 & 5 & 1000 & 124 & 39 & 34.70 & -71 & 3.50 & Y.T & 4116 \\
\hline 4 & 5 & 1100 & 124 & 38 & 23.90 & -70 & 58.70 & $\times B T$ & $\| 117$ \\
\hline 4 & 5 & 1200 & 124 & 38 & 13.30 & -70 & 54.60 & $\times R, r$ & 4119 \\
\hline 4 & 5 & 1300 & 124 & $3 R$ & 14.50 & -71 & $x, 6,0$ & $X \cap T$ & 4119 \\
\hline 4 & 5 & 1400 & 124 & 33 & 25.90 & -71 & 17.90 & $\times 3 \mathrm{~T}$ & 4120 \\
\hline 4 & 5 & 1500 & 124 & 39 & 31.30 & -71 & 32.30 & $\times 31$ & 7121 \\
\hline
\end{tabular}


TABLE 5: METEOROLOGICAL OBSERVATIONS

WARM CIRE RINGS METEOROLOGICAL LOG

30 SHIP: ENDEAVOR CRUISE: 83 CHIEF SCIENTIST: T. JOYCE DATES: 17 APR. $82-$ M MAY 92

40 GMT HINO HINO SHIP SHIP BAR. ORY HET SEA SHELL SHELL W WINO PRES.

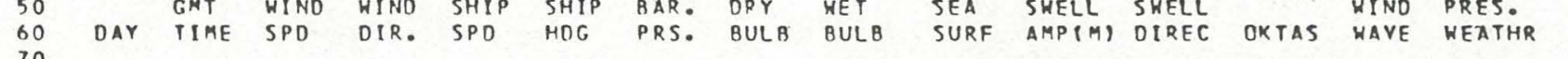

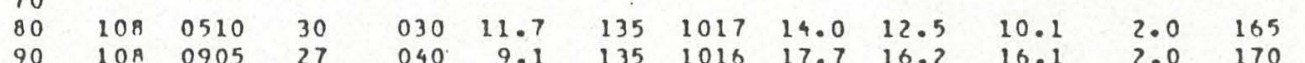

$\begin{array}{rrrrrrrrrrrrr}90 & 10 R & 0905 & 27 & 040 & 9.1 & 135 & 1016 & 17.7 & 16.2 & 16.1 & 2.0 & 170 \\ 100 & 108 & 1300 & 37 & 040 & 12.0 & 140 & 1016 & 20.0 & 18.8 & 20.5 & 2.0 & 190\end{array}$

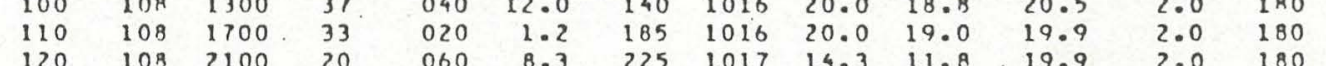

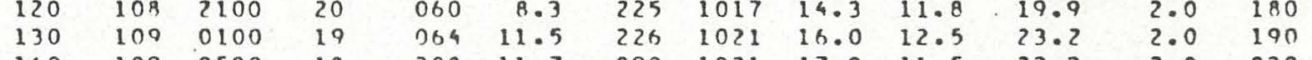

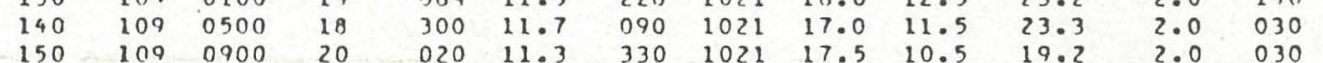

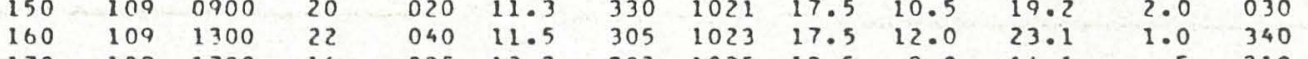

$\begin{array}{lllllllllllll}170 & 109 & 1700 & 16 & 025 & 12.3 & 282 & 1025 & 13.5 & 9.0 & 14.1 & .5 & 310\end{array}$

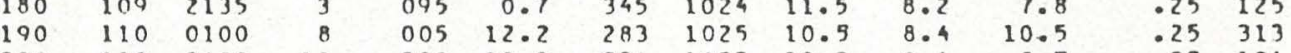

$\begin{array}{lllllllllllll}200 & 110 & 0400 & 11 & 320 & 12.0 & 236 & 1025 & 10.3 & 8.4 & 8.7 & .25 & 196\end{array}$

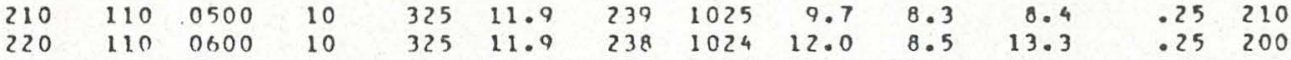

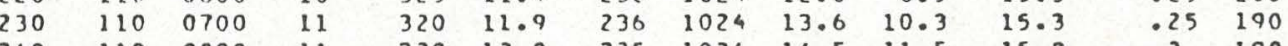

$\begin{array}{lllllllllllll}240 & 110 & 0800 & 11 & 320 & 12.0 & 235 & 1024 & 14.5 & 11.5 & .15 .8 & .2 & 190 \\ 250 & 110 & 0900 & 12 & 320 & 12.0 & 226 & 1023 & 14.7 & 10.5 & 15.5 & .2 & 090\end{array}$

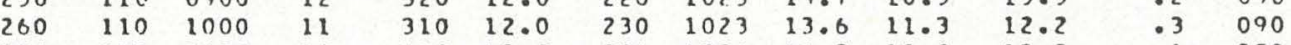

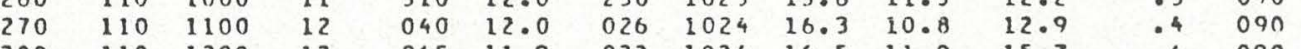

$\begin{array}{lllllllllllll}280 & 110 & 1200 & 13 & 045 & 11.9 & 022 & 1024 & 16.5 & 11.0 & 15.7 & .4 & 090\end{array}$

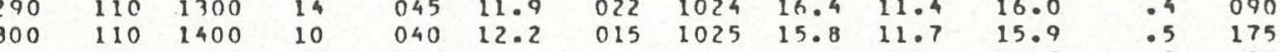

$\begin{array}{lllllllllll}310 & 110 & 1505 & 10 & 060 & 12.3 & 019 & 1025 & 15.0 & 12.0 & 13.7\end{array}$

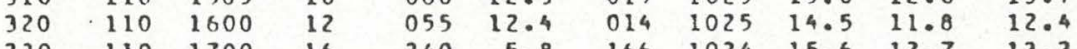

$110 \quad 1700$

$350 \quad 110 \quad 1900$

$\begin{array}{lll}350 & 110 & 2000 \\ 360 & 110 & 2100\end{array}$

$370 \quad 110 \quad 2200$

$\begin{array}{lll}380 & 110 & 2300 \\ 390 & 111 & 0000\end{array}$

$400 \quad 1110100$

$410 \quad 1110200$

$430 \quad 111 \quad 0400$

$\begin{array}{lll}440 & 111 & 0500 \\ 450 & 111 & 0600\end{array}$

$\begin{array}{lll}450 & 111 & 0600 \\ 460 & 111 & 0700\end{array}$

470 111 0 R०O

$490 \quad 111 \quad 1000$

$\begin{array}{lll}500 & 111 & 1100 \\ 510 & 111 & 1200\end{array}$

$\begin{array}{lll}510 & 111 & 1200 \\ 520 & 111 & 1300\end{array}$

$\begin{array}{llll}530 & 112 & 0500 & 22 \\ 540 & 112 & 0923 & 19\end{array}$

$\begin{array}{llll}550 & 112 & 1910 & 12\end{array}$

$\begin{array}{llll}570 & 112 & 2107 & 13 \\ 580 & 113 & 0000 & 24\end{array}$

$590 \quad 113 \quad 1345 \quad 15$

$\begin{array}{llll}610 & 113 & 2100 & 26\end{array}$

$\begin{array}{lllllll}340 & 5.8 & 166 & 1024 & 15.6 & 12.7 & 13.3\end{array}$

$\begin{array}{lllllll}345 & 11.8 & 165 & 1025 & 16.1 & 12.5 & 15.1 \\ 345 & 11.9 & 160 & 1024 & 16.6 & 12.7 & 15.0\end{array}$

$\begin{array}{lllllll}335 & 12.0 & 162 & 1024 & 15.5 & 12.0 & 16.1\end{array}$

$\begin{array}{lllllll}330 & 12.0 & 157 & 1024 & 14.0 & 11.5 & 12.7\end{array}$

$\begin{array}{lllllll}075 & 12.3 & 359 & 1023 & 14.0 & 11.4 & 10.5 \\ 060 & 12.3 & 312 & 1024 & 15.0 & 12.4 & 13.0\end{array}$

$\begin{array}{lllllll}060 & 12.3 & 312 & 1024 & 15.3 & 12.8 & 12.4\end{array}$

$\begin{array}{lllllll}140 & 12.2 & 311 & 1023 & 17.0 & 14.0 & 16.1 \\ 180 & 12.3 & 304 & 1021 & 17.1 & 14.6 & 16.1\end{array}$

$\begin{array}{lllllll}285 & 12.1 & 301 & 1022 & 18.3 & 15.1 & 15.5\end{array}$

$\begin{array}{lllllll}025 & 12.2 & 095 & 1023 & 16.8 & 14.5 & 12.7\end{array}$

$\begin{array}{lllllll}025 & 12.2 & 094 & 1021 & 17.6 & 15.5 & 15.7\end{array}$

$\begin{array}{lllllll}030 & 12.0 & 092 & 1021 & 17.7 & 15.6 & 15.9\end{array}$

$\begin{array}{lllllll}038 & 12.3 & 084 & 1020 & 14.5 & 12.6 & 12.7\end{array}$

$359 \quad 12.0$

084102014.512 .6

12.7
8.3

35512.3

$168,1020 \quad 13.3 \quad 11.6 \quad 9.9$

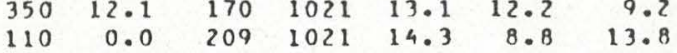

$\begin{array}{lllllll}115 & 0.0 & 253 & 1021 & 11.0 & 7.5 & 15.7\end{array}$

$\begin{array}{lllllll}060 & 0.6 & 210 & 1020 & 12.6 & 6.4 & 16.0 \\ 065 & 0.5 & 065 & 1019 & 12.2 & 7.5 & 16.0\end{array}$

$100 \quad 0.0$

$050 \quad 8.4$

$\begin{array}{lllll}065 & 1019 & 12.2 & 7.5 & 16.0\end{array}$

$060 \quad 12.2$

$\begin{array}{lllll}050 & 1022 & 7.6 & 4.0 & 10.8\end{array}$

$015 \quad 12.2$

$\begin{array}{lllll}194 & 1022 & 10.4 & 5.6 & 10.1\end{array}$

\begin{tabular}{l}
1 \\
1 \\
1 \\
8 \\
8 \\
5 \\
4 \\
2 \\
1 \\
0 \\
0 \\
0 \\
0 \\
0 \\
0 \\
0 \\
0 \\
0 \\
0 \\
0 \\
0 \\
2 \\
1 \\
2 \\
2 \\
1 \\
2 \\
4 \\
4 \\
4 \\
4 \\
4 \\
6 \\
6 \\
6 \\
6 \\
4 \\
4 \\
6 \\
7 \\
7 \\
7 \\
7 \\
0 \\
6 \\
4 \\
\hline 4 \\
4 \\
1 \\
\hline 1 \\
0 \\
- \\
\end{tabular}

$$
\begin{aligned}
& 1 \\
& 1 \\
& 1 \\
& 1 \\
& 1 \\
& 1 \\
& 1 \\
& .5 \\
& .2 \\
& .1 \\
& .1 \\
& 01 \\
& 01 \\
& 01 \\
& 01 \\
& 01 \\
& .1 \\
& .2 \\
& .1 \\
& .2 \\
& .1 \\
& .2 \\
& .2 \\
& .2 \\
& .2 \\
& .5 \\
& .5 \\
& .2 \\
& .2 \\
& .2 \\
& .1 \\
& .1 \\
& .1 \\
& .1 \\
& .1 \\
& .1 \\
& .1 \\
& .2 \\
& .2 \\
& .1 \\
& .2 \\
& .1 \\
& .1 \\
& .1 \\
& .1 \\
& .2 \\
& .1 \\
& .2 \\
& .2 \\
& .3 \\
& .3 \\
& .1 \\
& .1 \\
&
\end{aligned}
$$

$39,52.90-88,59.60$ $39.19 .00-68.12 .00$ $38.50 .40-67.32 .00$ $38,34.90-67,10.00$ $39.27 .00-67,16.00$ $38.00 .90-67,98.00$ $37,40.80-67,58.00$ $37,46.00 \quad-67,17.60$ $3 R, 22.10-67,50.10$ $38,54.80-68,40.20$ $39,59.00 \quad-69,22.60$ $39,01.30-69,28.70$ $30,09.70-70,16.50$ $39,02.80-70,29.00$ $39.55 .40-70,41.40$ $38.48 .00-70,54.90$ $3 R, 40.70-71,08.50$ $38,32.70-71,23.20$ $38,25.20-71,36.20$ $3 \times .34 .30-71.33 .30$ 3 म,46.10 $-71,28.90$ $32.58 .40-71.22 .40$ $39.09 .80-71,17.10$ $39.22 .90-71,11.30$ $30,33.20-71,06.60$ $29,29.54 \quad-71,01.39$ $39,17.20-70,54.60$ $39,05.20-71,49.80$ $34.52 .50-70.44 .60$ $39.39 .70-70.39 .40$ $38.27 .10-70,34.30$ $38.37 .90-70.43 .80$ $38.37 .80-70,55.90$ $7 R, 52.40-71.08 .20$ $38,59.80-71,34.80$ $39.06 .90-71,47.10$ $39,11.80 \quad-71,49.00$ $30,11.70-71,32.80$ $30,11.50-71,16.20$ $32,11.40 \quad-70,59.10$ $39,11.50-70,43.40$ $30,11.60-70,27.90$ $39,00.80-70,23.90$ $3 R, 50.00-70,23.00$ $38,43.59-70.51 .57$ $39.50 .00-71.02 .50$ $39,59.50-71,2 B .60$ $38,58.70-71,26.70$ $39,10.30-71,49.40$ $37,17.40 \quad-72,07.20$ $39,38.60-71.41 .10$ $30,45.20-71,21.50$ 
TABLE 5: METEOROLOGICAL OBSERVATIONS (Cont.)

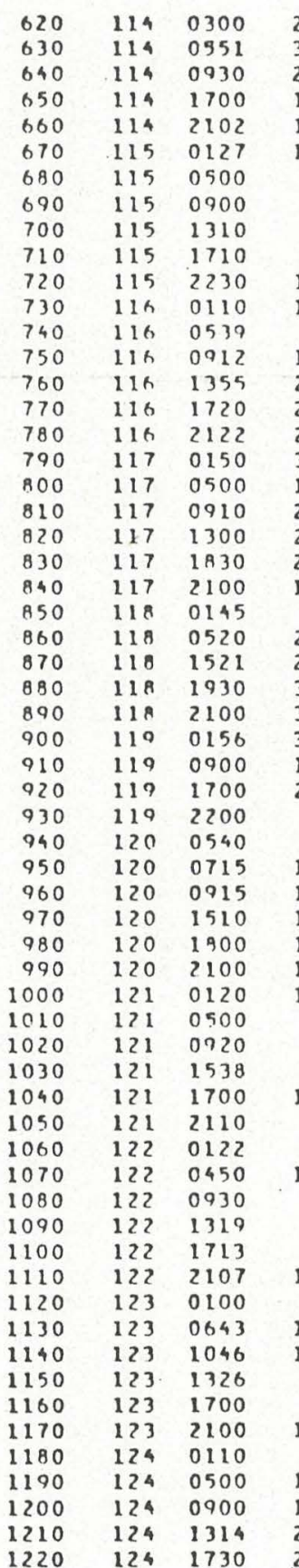

\begin{tabular}{|c|c|c|c|c|c|c|c|c|}
\hline 250 & 0.0 & - & 1021 & 16.7 & 11.5 & 15.4 & 1.0 & 275 \\
\hline 30 & 7.6 & 226 & 1020 & 16.0 & 11.5 & 15.0 & 1.0 & 270 \\
\hline 035 & 2.1 & 240 & 1020 & 15.5 & 11.0 & 15.7 & 1.0 & 240 \\
\hline 070 & 11.7 & 165 & 1022 & 17.4 & 12.2 & 15.0 & 1.0 & 270 \\
\hline 320 & 0.2 & 301 & 1022 & 17.0 & 12.3 & 16.0 & 1.2 & 270 \\
\hline 225 & - & 335 & 1028 & 15.5 & 12.0 & 15.3 & .5 & 300 \\
\hline 070 & 0.3 & 148 & 1024 & 15.0 & 12.2 & 13.1 & .5 & 270 \\
\hline 075 & 0.3 & 150 & 1025 & 12.0 & 10.0 & 10.0 & .3 & 230 \\
\hline 070 & - & 135 & 1026 & 12.5 & 10.0 & 8.8 & .2 & 235 \\
\hline 250 & 11.9 & 299 & 1026 & 15.0 & 12.1 & 14.6 & 1.1 & 250 \\
\hline$\triangle B O$ & 12.4 & 084 & 1025 & 14.7 & 12.5 & 11.6 & .7 & 200 \\
\hline 0 Oro & - & 132 & 1026 & 15.1 & 12.5 & 13.0 & .5 & 270 \\
\hline 070 & 0 & 110 & 1026 & 18.0 & 14.7 & 15.3 & .2 & 180 \\
\hline 050 & 11.2 & 082 & 1027 & 16.7 & 14.8 & 15.4 & .2 & ORO \\
\hline 045 & 11.9 & 092 & 1025 & 18.3 & 15.4 & 16.0 & 1.0 & 130 \\
\hline 060 & 12.0 & 084 & 1025 & 18.6 & 15.5 & 15.8 & 1.0 & 160 \\
\hline 135 & 1.0 & 138 & 1023 & 17.6 & 15.2 & 15.0 & 1.5 & 230 \\
\hline 345 & 7.5 & 190 & 1022 & 17.3 & 15.2 & 15.8 & 1.5 & 220 \\
\hline 090 & 0.4 & 085. & 1020 & 18.3 & 16.0 & 15.8 & 1.5 & 180 \\
\hline 105 & 0.3 & 310 & 1010 & 16.5 & 15.8 & 14.8 & 4.0 & 180 \\
\hline 010 & 0.3 & 170 & 1019 & 17.3 & 16.9 & 15.7 & 3.0 & 180 \\
\hline 140 & 4.2 & 033 & 1018 & 17.8 & 15.5 & 13.9 & 3.0 & 180 \\
\hline 025 & 0.0 & 185 & 1017 & 15.3 & 14.5 & 13.5 & 3.0 & 190 \\
\hline- & 0.0 & 083 & 1015 & 15.3 & 14.0 & 11.9 & 1.5 & 130 \\
\hline 320 & 11.6 & 252 & 1015 & 17.6 & 16.5 & 12.7 & 1.5 & 180 \\
\hline 350 & 1.7 & 027 & 1014 & 10.7 & 9.6 & 15.8 & 2 & 320 \\
\hline 010 & 6.0 & 010 & 1016 & 13.5 & 11.1 & 15.8 & 2.5 & 360 \\
\hline 360 & 1.7 & 020 & 1017 & 10.6 & 8.6 & 15.8 & 2 & 360 \\
\hline 020 & 6.3 & 355 & 1015 & ค. 0 & 7.0 & 15.8 & 2.5 & 360 \\
\hline 360 & 1.1 & 020 & 1021 & 11.0 & 7.3 & 13.0 & 1.5 & 030 \\
\hline 360 & 1.3 & 016 & 1024 & 11.0 & 7.2 & 13.2 & 1.0 & 360 \\
\hline 280 & 1.8 & 143 & 1024 & 11.5 & 6.5 & 14.0 & 1.0 & 045 \\
\hline- & 2.0 & 040 & 1025 & 11.8 & 6.0 & 14.5 & .5 & 270 \\
\hline 245 & 0.1 & 026 & 1024 & 12.6 & 9.5 & 14.3 & 125 & 270 \\
\hline 225 & 2.1 & 050 & 1024 & 12.5 & 8.8 & 13.3 & .3 & 290 \\
\hline 030 & 11.8 & .230 & 1024 & 14.0 & 9.5 & 15.7 & .5 & 270 \\
\hline 030 & 12.3 & 217 & 1023 & 15.0 & 12.0 & 14.7 & .5 & 270 \\
\hline 040 & 12.5 & 178 & 1023 & 12.3 & 10.0 & 10.2 & .5 & 270 \\
\hline 040 & 12.5 & 173 & 1024 & 14.2 & 11.4 & 12.8 & .2 & 300 \\
\hline 030 & 12.2 & 183 & 1024 & 16.4 & 12.5 & 20.2 & .2 & 270 \\
\hline 080 & 6.0 & 182 & 1022 & 19.5 & 11.8 & 23.4 & .2 & 090 \\
\hline 260 & - & 087 & 1024 & 20.0 & 12.5 & 21.2 & .2 & 060 \\
\hline 360 & 12.1 & $34 \mathrm{~A}$ & 1023 & 20.3 & 11.8 & 21.9 & .1 & 360 \\
\hline 125 & -.2 & 117 & 1022 & 22.0 & 13.5 & 24.0 & .1 & 120 \\
\hline 030 & 2.5 & 260 & 1022 & 21.3 & 14.0 & 24.8 & .1 & 045 \\
\hline 310 & 12.1 & 305 & $10 ? 2$ & 21.5 & 15.5 & 24.7 & .1 & 135 \\
\hline 075 & 0 & 185 & 1021 & 18.5 & 14.5 & 17.4 & .2 & 200 \\
\hline 232 & 0 & 132 & 1022 & 16.5 & 14.0 & 13.8 & .1 & 120 \\
\hline 110 & .2 & 135 & 1021 & 18.5 & 15.0 & 14.4 & .1 & 120 \\
\hline 090 & .3 & 155 & 1020 & 15.4 & 13.1 & 12.0 & .4 & 220 \\
\hline 060 & .4 & 168 & 1021 & 14.0 & 12.4 & 10.8 & b1 & - \\
\hline 320 & 12.0 & 342 & 1020 & 14.0 & 12.0 & 11.0 & 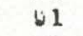 & - \\
\hline 100 & 12.0 & 342 & 1020 & 13.5 & 11.5 & 11.0 & .3 & 270 \\
\hline 090 & 0.2 & 175 & 1021 & 14.4 & 11.6 & 12.2 & .1 & 190 \\
\hline 060 & 12.1 & 110 & 1021 & 15.0 & 13.7 & 12.8 & .1 & 190 \\
\hline 305 & 11.9 & 021 & 1020 & 14.0 & 11.5 & 13.8 & .3 & 300 \\
\hline 000 & 12.1 & 015 & 1020 & 12.0 & 10.4 & 9.0 & .1 & 320 \\
\hline 020 & 12.1 & 160 & 1020 & 12.0 & 10.0 & 9.3 & .1 & - \\
\hline 030 & 11.9 & 158 & 1020 & 15.3 & 12.2 & 15.8 & .1 & 170 \\
\hline 030 & 12.0 & 305 & 1022 & 14.5 & 11.9 & 12.2 & .1 & 320 \\
\hline 20 & 12.1 & & 1023 & 16. & 11.5 & 15.4 & .1 & 325 \\
\hline
\end{tabular}

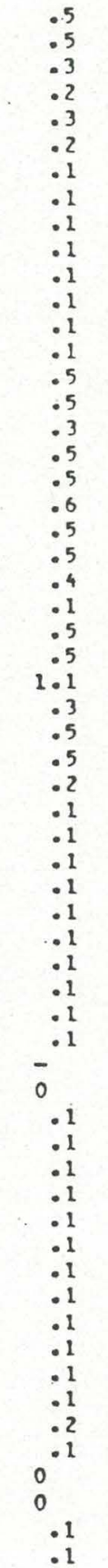

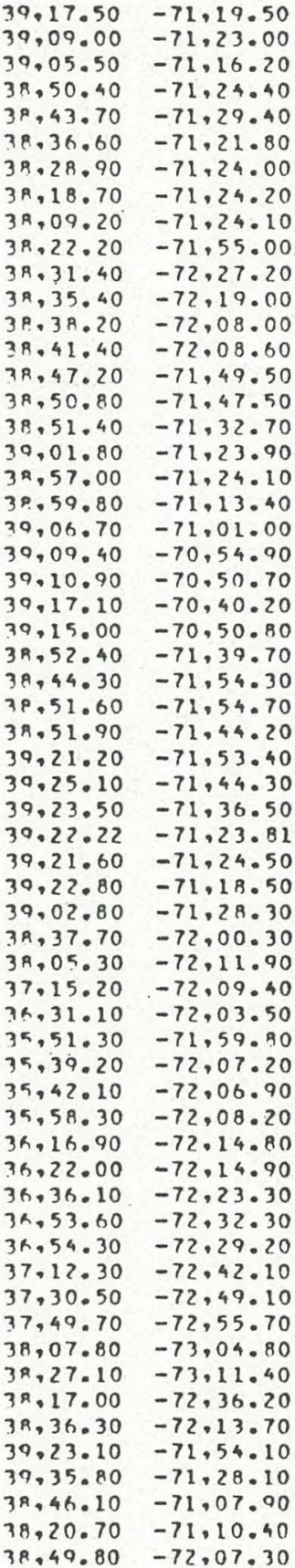




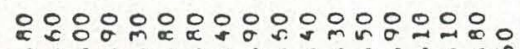

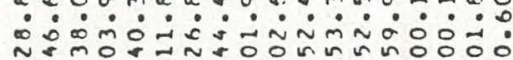

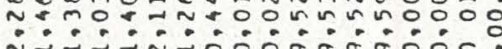

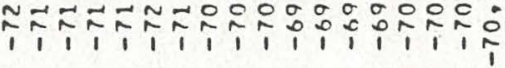

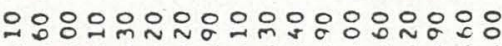

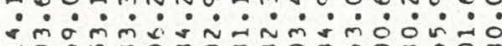
$\because 0 \%$ mom

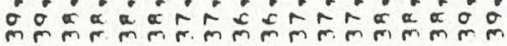

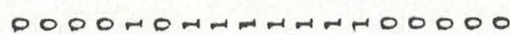

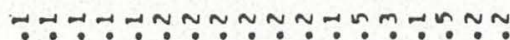

I 100m 10nmenmmommo

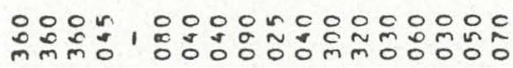

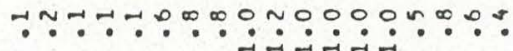

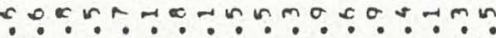

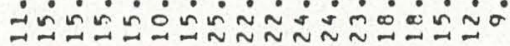

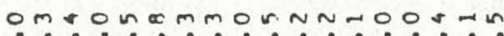

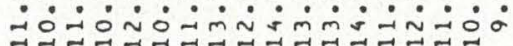
nnonomenomnosuomo

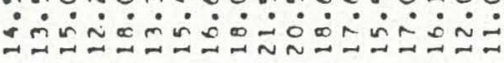

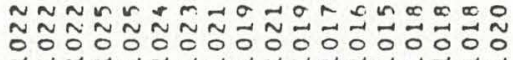

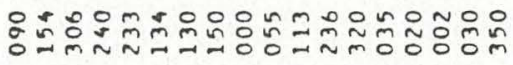

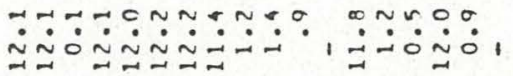

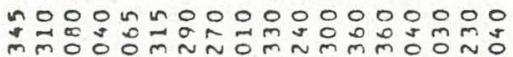

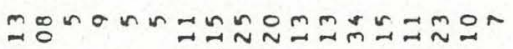

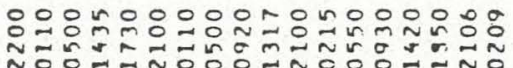

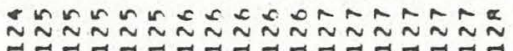

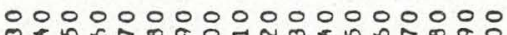

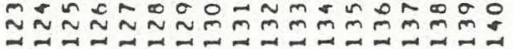




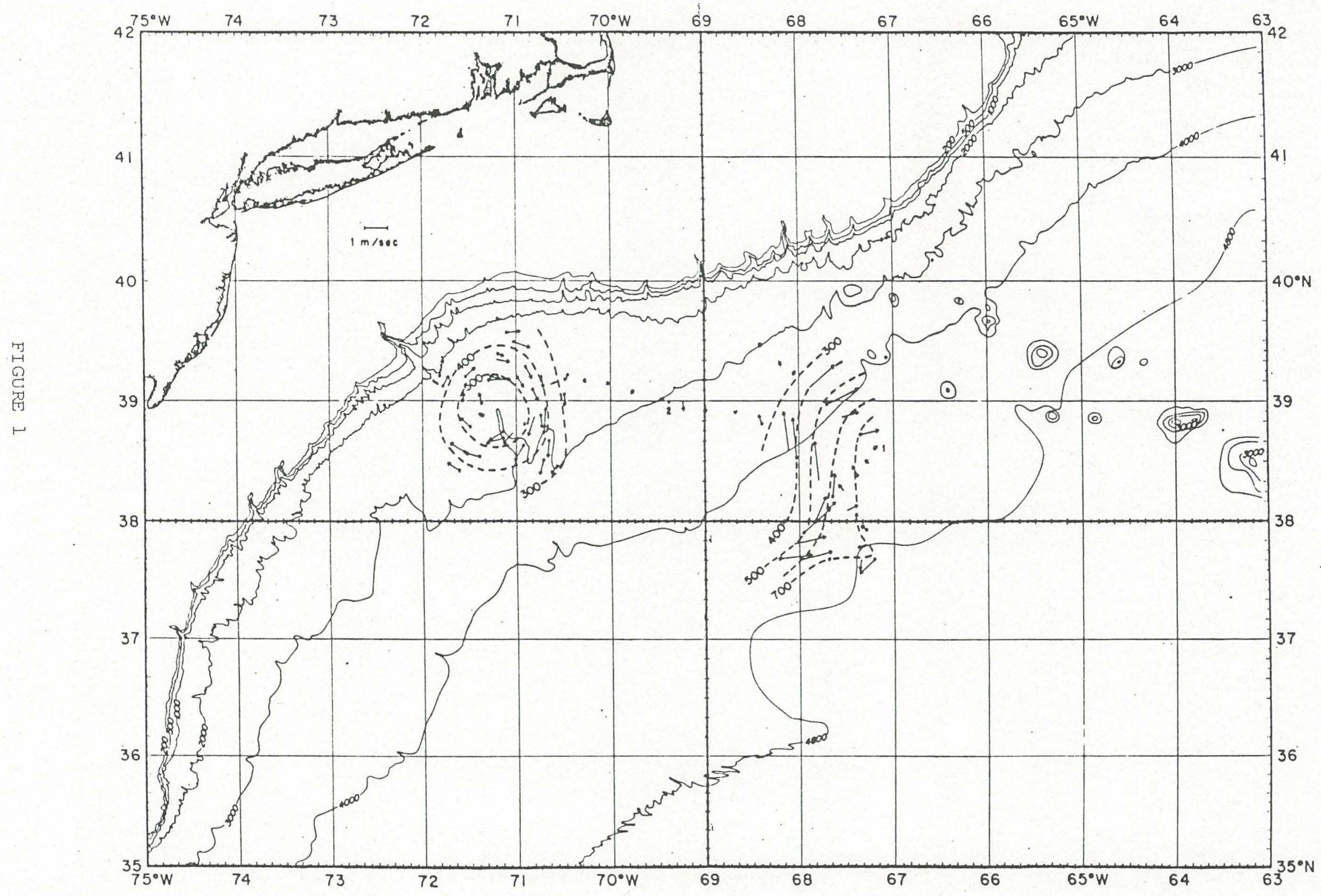




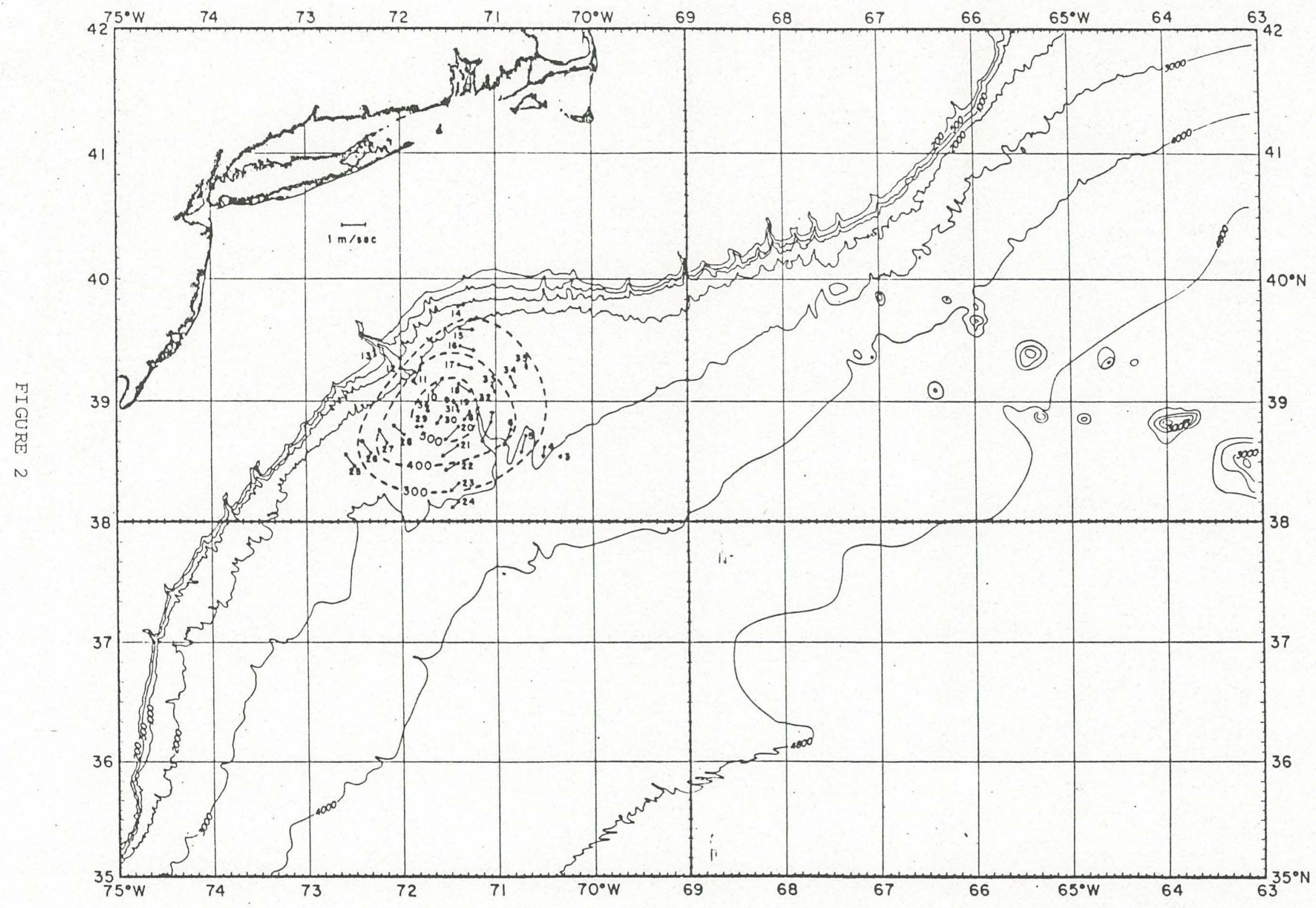




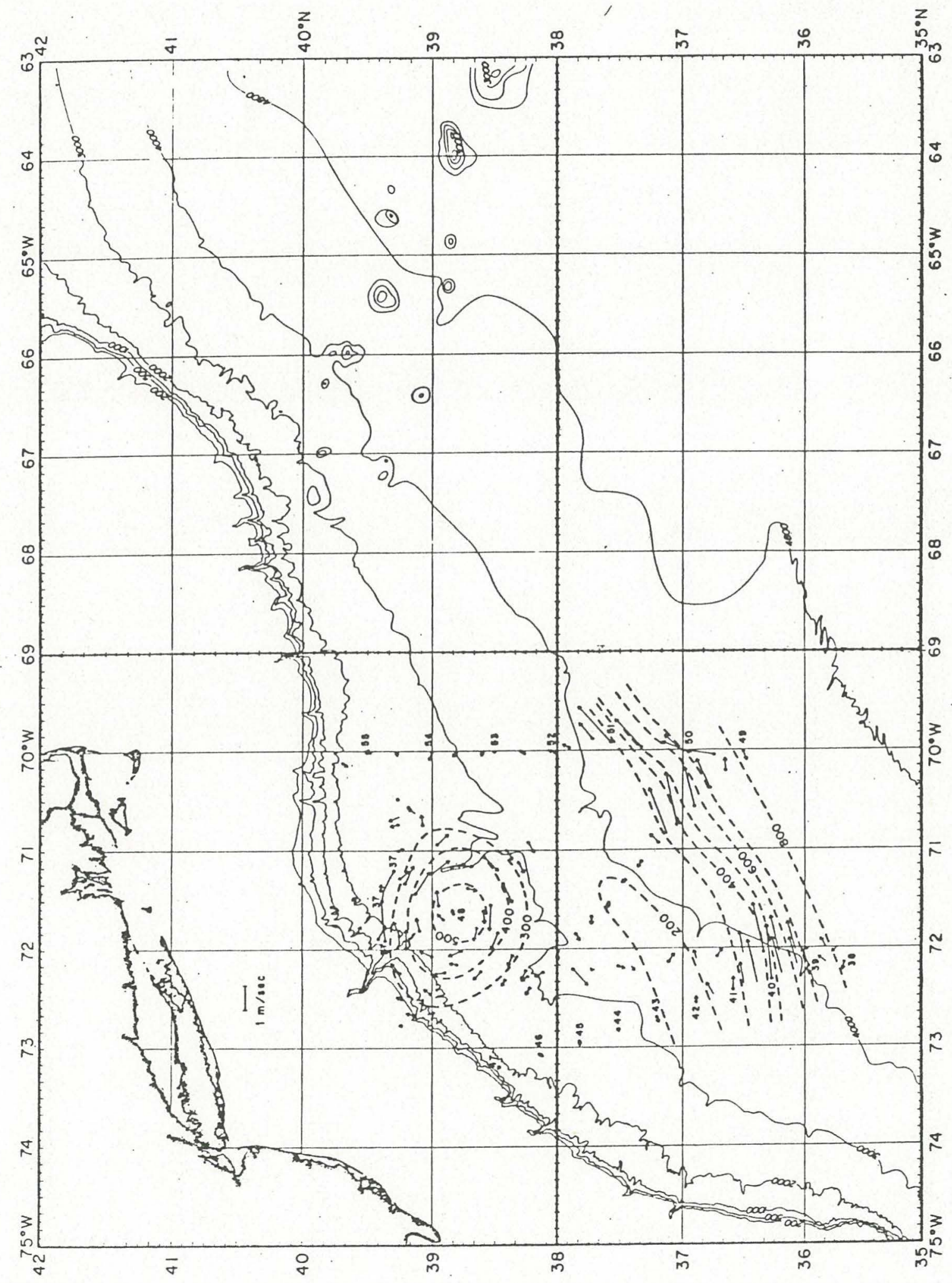

FIGURE 3 
$\stackrel{5}{5}$

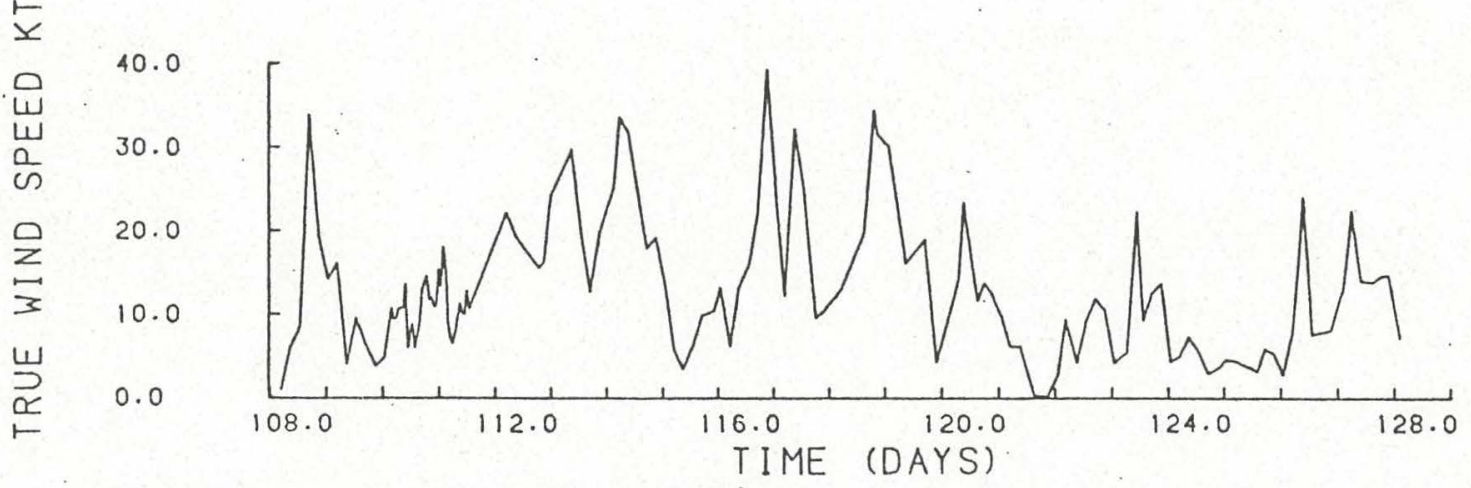

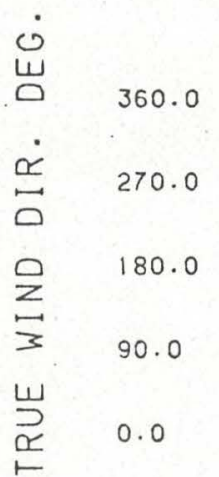

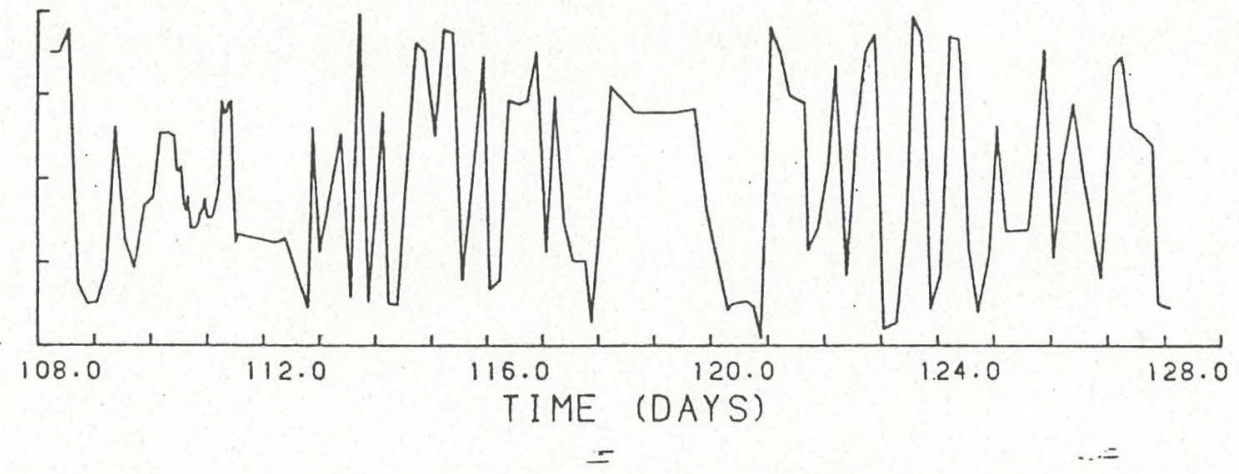

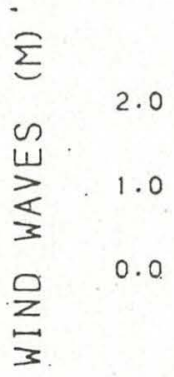

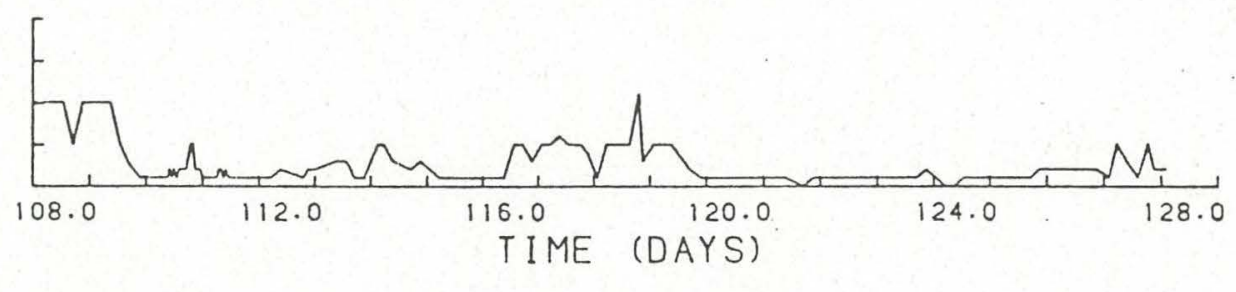

\begin{tabular}{ll} 
& 4.0 \\
$\sum$ & 3.0 \\
$\sum$ & 2.0 \\
\hline & 1.0 \\
$\frac{\omega}{3}$ & 0.0
\end{tabular}

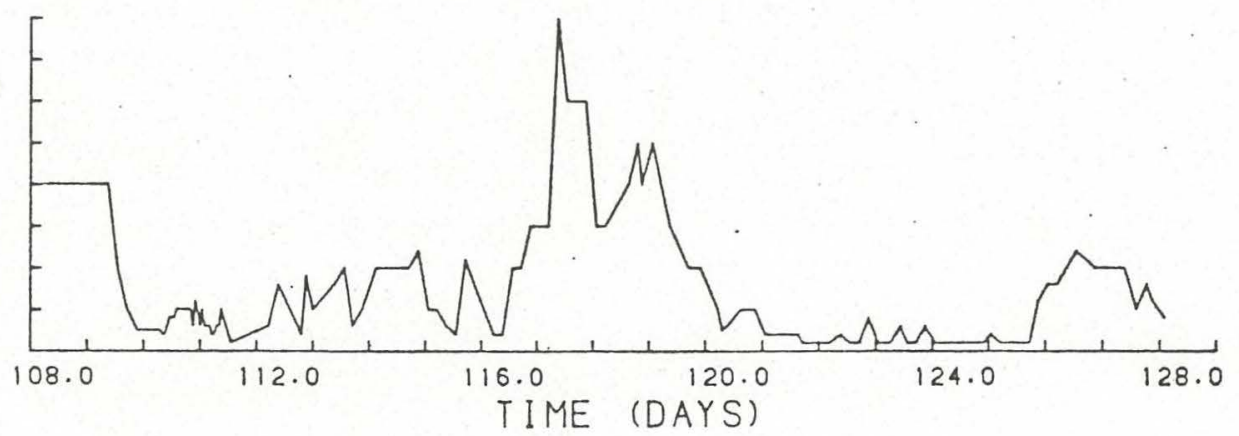


CRUISE REPORT

KNORR CRUISE NO. $93,4 / 19 / 82-5 / 6 / 82$

Dana R. Kester

University of Rhode Island

Narragansett, Rhode Island 


\section{KN-093 CRUISE REPORT}

\section{Objective of the Cruise}

This cruise was the first in a series of four during 1982 to investigate the physics, chemistry, and biology in Warm Core Rings north and west of the Gulf Stream in the Northwest Atlantic Ocean. This interdisciplinary investigation is a cooperative research program by more than 25 investigators funded by the National Science Foudation and by the National Aeronautics and Space Administration and by investigators with the National Marine Fisheries Service of NOAA.

The R/V KNORR was one of four vessels in this study. The other vessels were R/V ENDEAVOR, R/V OCEANUS, and the R/V ALBATROSS IV. At two times during this experiment a NASA P-3 aircraft flew over the region of study using remote sensors for sea surface temperature and spectral properties which could be related to similar shipboard measurements.

The activities aboard the R/V KNORR consisted of water column light intensity and quality and pigment analyses (Yentsch), phytoplankton species analysis (Fryxell), phytoplankton physiology and productivity (Smayda and Hitchcock), bacterial biomass and production (Ducklow), zooplankton grazing (Roman), zooplankton feeding and reproduction (Cowles), nutrient metabolism (McCarthy), silicon cycling (Nelson), nutrient distributions and CTD-oxygen profiling (Kester and Brown), and trace metal studies (Hanson and Kester).

\section{Cruise Itinerary}

19 April Depart Woods Hole, Massachusetts at 1130 am

20 April Arrive at Slope Water Station

22 April Arrive at Ring 81-B Center Station

24 April Conduct radial transect of ring

25 April Ri.. J Center Station

28 April Ring Center Multi-Ship Experiment

29 April High Velocity Region Multi-Ship Experiment

30 April Gulf Stream Station

1 May Sargasso Sea Station

2 May Gulf Stream Station

4 May Ring Center Station

5 May Slope Water Station

6 May Arrive at Woods Hole, Massachusetts at 1000 am

III. Surmary of Funded Investigations

Investigator Agency Grant

Cowles

Ducklow

NSF OCE-80-17271

NSF OCE $-81-17713$ 
$\begin{array}{ll}\text { Fryxell } & \text { NSF OCE-81-01785 } \\ \text { Hanson/Kester } & \text { NSF OCE-81-17848 } \\ \text { Kester/Brown } & \text { NSF OCE-80-22989 } \\ \text { McCarthy } & \text { NSF OCE-80-22990 } \\ \text { Nelson } & \text { NSF OCE-80-17269-01 } \\ \text { Roman } & \text { NSF OCE-81-17562 } \\ \text { Smayda/Hitchcock } & \text { NSF OCE-80-17272 } \\ \text { Yentsch } & \text { NASA grant NAG 6-17, Suppl. 1 from Wallops Flight Center }\end{array}$

IV. Scientific Party

Ms. Cara Adler

Mr. Mark Altabet

Mr. Peter Bates

Mr. Mark Brzezinski

Dr. Mimi Brown

Ms. Nancy Copley

Dr. Tim Cowles

$\mathrm{Mr}$. Tom Crook

Dr. Hugh Ducklow

Dr. Greta Fryxell

Dr. Miles Furnas

Ms. Anne Gauzens

Dr. Alfred Hanson

Ms. Suzanne Hill

Dr. Gary Hitchcock

Dr. Dana Kester

Mr. Ron Kroll

$\mathrm{Mr}$. Chris Langdon

Dr. James McCarthy

Dr. David Nelson

Mr. John Nevins

Mr. David Phinney

Mr. Frank Pickles

Dr. Michael Roman

Dr. Theodore Smayda

Dr. Charles Yentsch

Harvard University

Harvard University

University of Rhode Island

Oregon State University

University of Rhode Island

Woods Hole Oceanographic Institution

Woods Hole Oceanographic Institution

Woods Hole Oceanographic Institution

Lamont-Doherty Geological Observatory

Texas A. \& M. University

University of Rhode Island

University of Maryland

University of Rhode Island

Lamont-Doherty Geological Observatory

University of Rhode Island

University of Rhode Island, Chief Scientist

Woods Hole Oceanographic Institution

University of Rhode Island

Haryard University

Oregon State University

Harvard University

Bigelow Laboratory

University of Rhode Island

University of Maryland

University of Rhode Island

Bigelow Laboratory

V. Ship's Officers and Crew

\begin{tabular}{|c|c|c|c|}
\hline $\begin{array}{l}\text { chard J. Bowen, } \\
\text { nald A. Moller, } \\
\text { hn K. Sweet, Jr., } \\
\text { lvid H. Megathlin, } \\
\text { nest G. Smith, } \\
\text { rome M. Cotter, } \\
\text { ward R. Brodrick, } \\
\text { ank D. Tibbetts, }\end{array}$ & $\begin{array}{l}\text { Master } \\
\text { Chief Mate } \\
\text { 2nd Mate } \\
\text { 3rd Mate } \\
\text { Radio Officer } \\
\text { Bosun } \\
\text { AB }\end{array}$ & $\begin{array}{l}\text { Lawrence T. Moore, } \\
\text { Glenn L. Cox, } \\
\text { David S. Hayden, } \\
\text { John Hurder, } \\
\text { Harry Rougas, } \\
\text { Harry F. Clinton, } \\
\text { Herman Wagner, } \\
\text { Peter D. O'Reilly, }\end{array}$ & $\begin{array}{l}\text { Chief Engineer } \\
\text { 1st Asst Engr } \\
\text { 2nd Asst Engr } \\
\text { 3rd Asst Engr } \\
\text { Electrician } \\
\text { Oiler } \\
\text { Oiler } \\
\text { Oiler }\end{array}$ \\
\hline
\end{tabular}


John P. Kamataris, $A B$

Peter M. Flaherty, $A B$

Edward F. Graham OS

Tomas M. Macedo OS

Stephen W. Cotter, OS/Dayman
John M. Gassert, Steward

Gilberto R. Garcia Cook

Mitchell G. Barros Messman

Stephen F. Bates, Messman

VI. Scientific Narrative

Seventeen stations were occupied during this cruise. A station consisted of various types of sampling at a particular geographical or hydrographic location. Some of these stations required up to 48 hours to complete. The various sampling operations are summarized in Table 1, the operation log. An operation number was assigned to each sampling. This number had the form KNmmdd.ss, where $\mathrm{KN}$ designates KNORR, $\mathrm{mm}$ is the month (04), dd is the day (20), and ss is a sequence number which begins at 01 each day. Also given in Table 1 are the local times (hours and minutes) which were Eastern Standard Time prior to 25 April, and Eastern Daylight Time after 24 April. The station number and general location are given with CTR=Center, $E D G=E d g e$ of the core, HI VELOC=the high velocity region of the ring, STM=Stream, and SARG=Sargasso. The latitude and longitude are given in degrees and minutes. An investigator is identified with each operation, but in most cases several investigators were involved with each operation. The descriptor and comment columns are abbreviated items whose meaning is known principally to the investigators.

XBT's were taken during several periods of the cruise to define the temperature structure of the Ring." These are summarized in Table 2. All XBT's were Sippican T-7's giving profiles to greater than 750 meters. Table 2 gives the time, month and day (GMT) position, surface bucket temperature, and the depths in meters of three selected isotherms, 15, 10 and 6 degrees Celcius.

Yentsch and Phinney obtained 18 days of underway measurements of surface temperature, conductivity, chlorophyll, fucoxanthin, phycoerythrin, and light transmission. Vertical profiles of these properties were obtained at 11 locations by pumping to depths of 110 meters. Each vertical profile was accompanied by measurements of downwelling and upwelling sunlight in the upper 100 meters. Fifty discrete samples were measured for spectral and attenuation properties. There were 200 discrete chlorophyll analyses to calibrate the continuous fluorometer measurements.

Ducklow and Hill collected samples for bacterial abundance and growth rate estimates from all of the productivity casts and from several silica deep and radial section casts. The preliminary results indicate a bacterial abundance maximum in the high velocity region. The bacterial abundance and thymidine uptake were constant with depth in the upper 100 meters at the ring center. 
Fryxell collected a series of samples through the water column to at least the $0.1 \%$ surface light intensity. These samples will be used to characterize the early spring flora from the Slope Water, Ring Center, High Velocity Region, and the Sargasso Sea. The $1 / 4$ meter MOCNESS sampling system was equipped with $37 \mathrm{um}$ mesh nets to obtained depth specific samples for phytoplankton analysis at six locations. Net tows (10 vertical and 5 horizontal) were taken to provide living material and material for light and electron microscopy. These samples showed the presence of gelatinous colonies of the diatom Thalassiosira. Dinoflagellates dominated the Slope Water station and they were well represnted during the initial sampling of ring center; large chain-forming diatoms were also present. The Sargasso Sea station showed the chain-forming diatom Chaetoceros. During the last visit to ring center there was an increased number of diatoms and gelatinous colonies, which is usually associated with upwelling areas.

Smayda, Hitchcock, Langdon, and Furnas carried out work to establish the rates of phytoplankton primary production, euphotic zone abundance of phytoplankton carbon, growth rate measurements of key species, and the vertical distribution, composition, and abundance of various filtration defined size classes of phytoplankton. These studies were carried out at nine primary productivity stations at nine depths between the $100 \%$ and $0.1 \%$ isolumes. The chlorophyll and ATP carbon content of these populations were determined. Preliminary data analysis reveals predominance of the < $10 \mathrm{um}$ size class with a variable contribution of tile $>64 \mathrm{um}$ size class. Six growth rate experiments were conducted for periods of 24 to 48 hours, evaluating the responses of key species. Eleven 1/4 meter MOCNESS tows using the $64 \mathrm{um}$ net provided samples at $25 \mathrm{~m}$ intervals in the upper $200 \mathrm{~m}$. The shipboard analysis revealed considerable populations of phytoplankton below the euphotic zone with a dramatic patch of the large diatom Cosinodiscus wailesii, heavily infected by the fungus Lagenidium.

Nelson and Brzezinski collected a series of samples and performed incubations to assess silicon cycling. Profiles were obtained for total and. amorphous particulate silica from 0 to $600 \mathrm{~m}$. Samples were obtained to determine biogenic silica production rates, and silica dissolution rates. Day-night station pairs were obtained in the Slope Water (20-21 April), at ring center (22-23 April, 26-27 April and 3-4 May), in the high velocity region (29-30 April) and in the Sargasso Sea (1-2 May). A series of radial stations from ring center to the high velocity region were obtained and one cast was made in the Gulf Stream.

McCarthy and coworkers measured phosphorus and nitrogen incorporation rates using $\mathrm{N}-15$ and $\mathrm{P}-33$ labelled substrates of nitrate, ammonium, urea, and phosphate. These experiments were conducted at six depths from $100 \%$ to $3 \%$ surface light intensity at first light (FL), mid-day (MD), and night-time (NT). This work was performed at the Slope Water, the Ring Center, the high velocity region and in the Sargasso Sea. 
Roman carried out a series of zooplankton studies using the $1 / 4$ meter MOCNESS sampling system, net tows; and in situ incubations. These measurements provided information on zooplankton grazing, size fractionation, and chemical composition of particulate material (carbon, nitrogen, chlorophyll, protein, carbohydrate, and lipids). This work was conducted in the slope Water, Ring Center, High Velocity Region, and in the Sargasso Sea. There were $101 / 4$ MOCNESS tows to $200 \mathrm{~m} ; 1$ tow to $1000 \mathrm{~m}$; and 2 toyos to $100 \mathrm{~m}$. Fourteen shipboard grazing experiments were conducted and seven in situ grazing experiments were performed. There were about 50 particulate chlorophyll, corganic carbon, and nitrogen measurements. There were 36 measurements of protein, carbohydrates and lipids, and 28 bacterial counts.

Kester and Brown carried out water column CTD-oxygen profiling and nutrient sampling. Two CTD rosette sampling systems were used: a 5L 24 bottle rosette, and a 30L 12 bottle rosette. There were four types of CTD casts: deep, which covered most or all of the water column; pump, which went to about $110 \mathrm{~m}$ with Yentsch's pump hose and fluorescence profiling; productivity casts to selected light intensity levels in the euphotic zone; and silica deep casts to $600 \mathrm{~m}$. There were 12 deep CTD's with the 24 bottle rosette and 6 deep CTD's with the $30 \mathrm{~L}$ rosette. Nutrient samples were analyzed onboard for nitrate, nitrite, phosphate, and silicate from the deep CTD's, the silica deep casts, the productivity casts, and from selected pump profiling casts. About 650 nutrient samples were analyzed, and another 60 analyses were performed on samples from experiments of various investigators.

Kester and Hanson conducted a series of trace metal investigations. A clean portable laboratory was used aboard the ship for nearly real-time analyses of trace metals by atomic absorption spectroscopy. An automated preconcentration system was used to extract copper, cadmium and nickel from seawater. Particular ittention was given to copper and its speciation. Several different chemical fractions of copper were isolated and analyzed. These included total (particulate, dissolved, organic and inorganic), dissolved, and organically complexed copper. The $\mathrm{pH}$ and alkalinity were measured to permit thermodynamic calculations of copper speciation based on our laboratory studies. Samples were obtained from 14 productivity casts and 9 deep CTD casts. There were 273 measurements of organically complexed copper and 63 anlyses of dissolved copper completed onboard ship. Other samples (447) were preserved for analysis onshore.

Tim Cowles carried out measurements of feeding response and reproductive potential of large copepods within and around the ring. He also collected zooplankton samples with the $1 / 4$ MOCNESS 
for microzooplankton analysis in collaboration with Mike Roman. Seven water column particle profiles were obtained; fifteen feeding experiments and egg production experiments were conducted; and $121 / 4$ MOCNESS tows were completed. The center of Ring 82-B contained a mixture of warm and cold water macrozooplankton. The surface waters contained Calanus finmarchicus during the day, while night tows revealed the cold water species of the genus Pleuromamma, that are not usually found in the Sargasso Sea. The ring center fauna were similar during collections on April 23, 26, and 28, but the May 2-3 sampling indicated a shift in copepod species composition. The data will be analyzed to determine if the animals are exploiting the available particulate matter in the ring so that both cold and warm water species have adequate food and maitain population recruitment rates. 
TABLE 1

KN-093 OPERATION LOG

$15 / 05 / 82$

IP. NO. TIME STA LOCATION LAT. LONG. INVSTGR DESCRIPT CORMENT

W0420.01 $0350 \quad 1 \quad$ SLOPE 3908.66924 .9 KESTER 5L DEEPCTD CTD 1

NO420.02 $0630 \quad \cdots 1$ SLOPE 3908.96925 .8 KESTER 30L CTD CTD

SNO420.04 0925 1 SLOPE 3909.36925 .3 FRYXELL VERTNET

NO $420.051100 \quad 1 \quad$ SLOPE 3908.76925 .5 YENTSCH TOTAL IRRAD

KNO420.06 $1150 \quad 1$ SLOPE 3908.76926 .0 YENTSCH 4 CHAN LITE

KNO420.07 $1301 \quad 1 \quad$ SLOPE 3908.76925 .7 COHLES 1/4 MOC TOW

KNO420.08 $1458: 1$ SLOPE 3905.06929 .0 FRYXELL 1/4 MOC

KNO420.09 $1630 \quad 1 \quad$ SLOPE $\quad 3909.06926 .0$ YENTSCH

KNO420.10 $1830 \quad 1 \quad$ SLOPE $\quad 3909.36924 .7$ MCCARTHY

KNO420.11 $1930 \quad 1 \quad$ SLOPE $\quad 3909.06925 .2$ COWILES

$\mathrm{KM} 0420.122032$

KNO420.132135

KNO420.14 2230

KN0420.030900

KNO421.010000

KNO421.02 0030

KNO421.03 0510

KH:0421.04 0556

KMO421.05 0700

KHO421.06 0830

KN0421.07.0900

KNO421.08 1342

KNO421.09 1430

KN0422.01 0530

KN0422.02 0800

KNO422.03 0835

KNO422.04 0915

KNO422.05 1100

KNO422.06 1200

KNO422.07 1330

SLOPE $\quad 3909.06924 .9$ COVILES

PUMP PROFILE CTD非3

PMPROD CTD 1 L 4

LIVE NET TOW 64 UM NET

$1 / 4 \mathrm{MOC}$ TOW $0-20064$ UM

$1 / 4$ MOC TOW 0-22M 37 UM

NTPROD CTD

MASA FLYOVER SURFACE CBS

LIVE NET TOW

5L DEEPCTD CTD $\# 7$

FLPROD CTD \#8 \& \#9

FLPROD CTD师9

SIDEEP CTDir 10--NG

VERTNET

LIVE NET TOW 64 UM NET

MDPROD 3OL HYDRO

LIVE NET TOW O-100M 2OUM

5L DEEPCTD CTD非11

VERTNET

LIVE NET TOW 64 UM NET

LIVE NET TOW

TOTAL IRRAD AT BUOY

4 CHAN LITE AT BUOY

PUMP PROFILE CTD留.12

ITEMS THIS PAGE 30 ITEMS THIS REPORT 30 
TABLE 1 (continued) KN-093 OPERATION LOG

$05 / 05 / 82$

\begin{tabular}{|c|c|c|c|c|c|c|c|c|}
\hline P. NO. & TIME & STA & LOCATION & LAT. & LONG. & INVSTGR & DESCRIPT & COMMENT \\
\hline KNO422.08 & 1510 & 2 & RING CTR & $38 \quad 49.7$ & $71 \quad 18.5$ & COWLES & $1 / 4$ MOC TOW & $0-200 M \quad 64 U M$ \\
\hline N0422.09 & 1550 & 2 & RING CTR & 3847.2 & 7120.8 & FRYXELL & $1 / 4 \mathrm{MOC}$ TOW & $0-35 \mathrm{M} 37 \mathrm{UM}$ \\
\hline N0422. 10 & 1840 & 2 & RING CTR & 3848.8 & $71 \cdot 22.8$ & SMAYDA & 1/2M NET TOW & 200-OH 35UM \\
\hline NNO422. 11 & 2042 & 2 & RING CTR & 3846.6 & 7123.9 & RON.AN & 3OL HYDRO & $1 \mathrm{BOT} 15 \mathrm{M}$ \\
\hline NO422. 12 & 2054 & 2 & RING CTR & 3846.6 & 7124.1 & COVILES & LIVE NET TOW & $200 \mathrm{M}$ \\
\hline N0422.13 & 2200 & 2 & RING CTR & 3847.0 & 7125.5 & MCCARTHY & NTPROD & CTD\#13 \\
\hline 10422.14 & 2245 & 2 & RING CTR & 3846.6 & 7127.0 & NELSON & SIDEEP & CTD米 14 \\
\hline 10422.15 & 2335 & 2 & RING CTR & 3846.7 & 7128.0 & COWLES & $1 / 4$ MOC TOW & $0-200 M$ \\
\hline 423.01 & 0650 & 2 & RING CTR & 3842.7 & 7134.6 & MCCARTHY & FLPROD非 1 & CTD非 15 \\
\hline No423.02 & 0723 & 2 & RING CTR & 3842.7 & 7134.6 & MCCARTHY & FLPROD fir2 & CTD俳 16 \& 非17 \\
\hline N0423.03 & 0810 & 2 & RING CTR & $38 \quad 42.8$ & 7135.1 & NELSON & SIDEEP & CTD\#18 \\
\hline NN0423.04 & 0915 & 2 & RING CTR & $38 \quad 44.1$ & 7137.8 & FRYXELL & $1 / 2 \mathrm{M}$ NET & $200-O M$ \\
\hline N0423.05 & 1032 & 2 & RING CTR & $38 \quad 42.1$ & 7137.4 & RONIAN & IN SITU INCU & \\
\hline N0423.06 & 1300 & 2 & RING CTR & $38 \quad 42.8$ & 7138.8 & MCCARTHY & MDPROD非 1 & \\
\hline 0423.07 & 1338 & 2 & RING CTR & 3842.8 & 7138.8 & MCCARTHY & MDPROD非2 & \\
\hline 10423.08 & 1400 & 2 & RING CTR & 3843.1 & 7140.1 & ROMAN & OBLIQUE NET & \\
\hline N0423.09 & 1500 & 2 & RING CTR & 3842.5 & 7140.1 & KESTER & 5L DEEPCTD & CTD \#19 \\
\hline 10423.10 & 2330 & 3 & RADIAL & 3927.7 & 7131.2 & FRYXELL & SURF NET TOW & BIOLUMINESC \\
\hline N0424.01 & 0024 & & RADIAL & 3928.1 & 7129.9 & YENTSCH & PUIPP PROFILE & CTD 俳 20 \\
\hline NO424.02 & 0300 & 3 & RADIAL & 3927.8 & 7130.9 & BRZEZINSKI & SIDEEP & CTD壮21 \\
\hline .03 & 0523 & 4 & RADIAL & 3942.2 & 7128.0 & BRZEZINSKI & SIDEEP & CTD H222 \\
\hline KNO42 & 0630 & 4 & RADIAL & 3942.6 & 7127.9 & FRYXELL & SURF NET TOW & LPG WIRE AGL \\
\hline $\mathrm{KNO} 4 \mathrm{z}$ & 0800 & 4 & RADIAL & 3953.7 & 7126.0 & BRZEZINSKI & SIDEEP & CTD非23 \\
\hline $\mathrm{KNO} 42$ & 1000 & 4 & RADIAL & 3953.7 & 7126.2 & FRYXELL & VERTNET & $0-200 \mathrm{M}$ \\
\hline KNO42 & 1114 & 6 & RADIAL & 3946.4 & 7126.9 & YENTSCH & PUMP PFOFILE & CTD壮24 \\
\hline $\mathrm{KNO} 42$ & 1608 & 6 & RADIAL & 3917.8 & 7131.0 & NELSON & SIDEEP & CTD壮25 AERTD \\
\hline $\mathrm{KNO} 42$ & 1910 & 8 & RADIAL & 3903.9 & 7134.0 & NELSON & SIDEEP & CTD非26 \\
\hline & 2200 & 8 & RADIAL & 3903. & 7134.0 & ROMAN & VERTNET & \\
\hline F.170 & 2230 & 8 & RADIAL & 3903.9 & 7134.0 & RON.AN & 30L HYDRO & 2 EOTTLES \\
\hline 4 & 0804 & 9 & RING CTR & 3900.0 & 7130.0 & ROMAN & LIVE NET TOW & \\
\hline
\end{tabular}

ITEMS THIS PAGE 30 ITEMS THIS REPORT 60 
TABLE 1 (continued) KN-093 OPERATION LOG

$05 / 05 / 82$

OP. NO. TIME STA LOCATION LAT. LONG. INVSTGR DESCRIPT COMMENT

KNO425.02 0930

$\mathrm{KNO} 025.031030$

KNO425.05 1150

KNO425.06 1400

KNO425.07 1430

KNO425.08 1444

KN0425.09 1600

KNO425.10 1700

KNO425.11 1804

KNO4 25.122045

KNO425.132050

KNO425.14 2230

KNO426.01 0505

KNO426.02 0700

$\mathrm{KNO} 426.030900$

KNO426.04 0950

KNO426.06 1200

KNO426.07 1330

KNNO426.08 1400

KNO426.09 1500

KNO426.10 1845

KNO426.11 2033

KNO426.12 2044

KNO426.132200

KNO427.01 0510

KNO427.02 0915

KNO427.03 1225

$\mathrm{KNO} 427.041300$

KNO 427.051510

KNO427.06 2030

9

RING CTR 3859.47132 .3 FRYXELL

$9 \quad$ RING CTR 3859.77131 .4 DUCKLOW

$9 \quad$ RING CTR 3859.47131 .4 COWLES

9 RING CTR 3855.57128 .5 FURNAS

9 RING CTR 3854.97129 .0 ALTABET

9 RING CTR 3854.27129 .6 ALTABET

9 RING CTR 3900.27129 .8 YENTSCH

9 RING CTR 3859.071 27.7 FURNAS

9 RING CTR 3900.07130 .1 KESTER

9

RING CIR 3859.27129 .7 RONAN

$9 \quad$ RING CTR 3859.27129 .7 COVLES

9 RING CTR 3858.67129 .7 BROWN

9 RING CTR 3900.17129 .8 MCCARTHY

9 RING CTR $3859.871 \quad 29.8$ NELSON

9 RING CTR 3859.87130 .0 FRYXELL

9 RING CTR $3859.871 \quad 30.0$ ROMAN

9 RING CTR $3900.271 \quad 29.7$ MCCARTHY

9 RING CTR 3900.27129 .7 DUCKLOW

9 RING CTR 3900.27129 .7 YENTSCH

$9 \quad$ RING CTR $3900.271 \quad 29.7$ ROMAN

9 RING CTR 3854.27121 .8 FRYXELL

9. RING CTR 3858.97128 .2 CONLES

9 RING CTR 3858.97128 .3 COWLES

$9 \quad$ RING CTR 3859.17128 .6 MCCARTHY

10 RING EDG $28 \quad 41.87106 .8$ KESTER

10 RING EDG $3845.671 \quad 18.0$ KESTER

10 RING EDG 3842.07120 .5 COWLES

10. RING EDG $38 \quad 41.27121 .3$ KESTER.

10 RING EDG 3842.97126 .8 COWLES

11 RING CTR 3854.97137 .4 FURNAS
HORZNET 1OY
3OL NISKIN
$1 / 4$ HOC TOW TOYO
LIVE NET TCW 200M
LIVE NET TOW 15i
LIVE NET TOW $75 \mathrm{M}$
PLMP PROFILE CTD 范7 .
LIVE NET TOW 150M
$5 L$ DEEPCTD CTD非8
3OL HYDRO 1 BOTTLE $5 \mathrm{H}$
LIVE NET TOW OBLIQUE NEI
3OL DEEPCTD CTD\#29
FLPROD CTD\#30\& \#31
SIDEEP CTDi32
VERTNET 200 TO OM
INSITU INCUB 0 TO $68 \mathrm{M}$
MDPROD CTD非33 \& \#34
3OL HYDRO TO $110 \mathrm{M}$
TOTAL IRRAD
1/4MOC TOW TO 1000M
1/4MOC TOW PHYTO NETS
3OL HYDRO $5 \& 7 \mathrm{M}$
LIVE NET TOW TO $100 \mathrm{M}$
HTPROD CTD范35\&36
30L DEEPCTD CTD
5L DEEPCTD CTD非38
LIVE NET TOW O TO 100M
5L CTD/YENCH CTD\#39
$1 / 4 M O C$ TOW TOVO
3OL HYDRO O $2550 M$ 
TABLE 1 (continued) KN-093 OPERATION LOG

$05 / 05 / 82$

OP. NO. TIME STA LOCATION LAT. LONG. INVSTGR DESCRIPT COMNENT

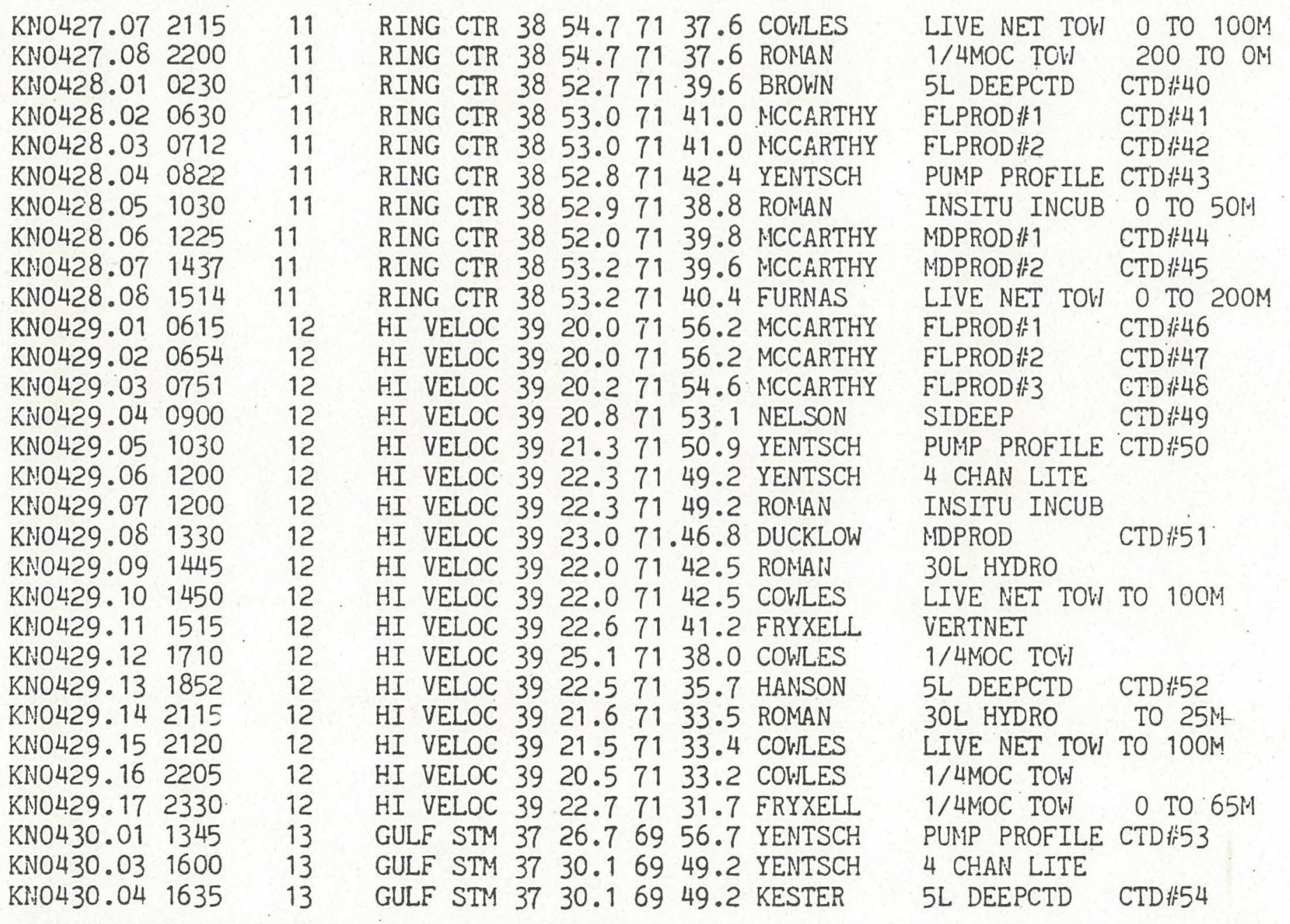

ITEMS THIS PAGE 30 ITEMS THIS REPORT 120 
TABLE 1 (continued) KN-093 OPERATION LOG

$05 / 05 / 82$

OP. NO. TIME STA LOCATION LAT. LONG. INVSTGR DESCRIPT COMAENT

KN0501.01 0435 14 SARG SEA 3641.76925 .3 FRYXELL

KN0501.02 0520 $14 \quad$ SARG SEA $36 \quad 42.5 \quad 6924.7$ DUCKLOW

KN0501.03 $0625 \quad 14 \quad$ SARG SEA 3642.76924 .9 DUCKLOW

KN0501.04 $0730 \quad 14 \quad$ SARG SEA 3642.76924 .1 NELSON

KN0501.05 $0852 \quad 14 \quad$ SARG SEA $36 \quad 43.86921 .8$ YENTSCH

KN0501.06 $1045 \quad 14$. SARG SEA $36 \quad 44.56921 .8$ YENTSCH

KN0501.07 1050 14 SARG SEA $36 \quad 45.769$ 20.0 ROMAN

KN0501.08 $1210 \quad 14$

KN0501.09 $1249 \cdot 14$

KN0501.10 $1300 \quad 14$

KN0501.11 1305 14

KN0501.12 $1412 \quad 14$

KN0501.13 $1542 \quad 14$

KNO501.14 $1700 \quad 14$

KN0501.15 2215 14

KN0501.16 2246 14

KN0501.17 $2340 \quad 14$

KN $0502.010015 \quad 14$

KN0502.02 $0100 \cdot 14$

KNN0502.03 0800

KN0502.04 2030

KN0502.05 2040

KN10502.06 2110

KN0503.010600

SARG SEA $36 \quad 45.7 \quad 6919.9$ COWLES

SARG SEA $36 \quad 45.7 \quad 69.19 .9$ FRYXELL

SARG SEA 3647.36919 .0 MCCARTHY

SARG SEA $36 \quad 47.3 \quad 69 \quad 19.0$ YENTSCH

SARG SEA $36 \quad 47.7 \quad 69 \quad 17.8$ COWLES

SARG SEA $36 \quad 45.569 \quad 18.4$ FRYXELL

SARG SEA $36 \quad 42.76924 .4$ KESTER

KN0503.02 0630

KN0503.03 0730

KN 0503.040840

KN 0503.050945

SARG SEA $36 \quad 42.56924 .3$ BRZEZINSKI

SARG SEA $36 \quad 42.56924 .3$ KESTER

SARG SEA $36 \quad 42.4 \quad 6924.4$ R.OMAN

SARG SEA $36 \quad 42.56924 .6$ COVILES

SARG SEA $36 \quad 42.56925 .7$ COWLES

GULF STM $37 \quad 14.3 \quad 7031.9$ KESTER

RING CTR 3857.57140 .1 COWLES

RING CTR $38 \quad 57.471 \quad 40.2$ COWLES

RING CTR 3857.4 .71 40.8 COVILES

RING CTR $3857.871 \quad 40.0$ FRYXELL

RING CTR $3858.271 \quad 40.2$ FRYXELL

RING CTR $3858.271 \quad 40.2$ ROMAN

RING CTR 3858.071 40.1 FURNAS

RING CTR 3858.17140 .1 YENTSCH

RING CTR $38 \quad 58.471 \quad 37.4$ YENTSCH

RING CTR 3858.07137 .6 COWLES

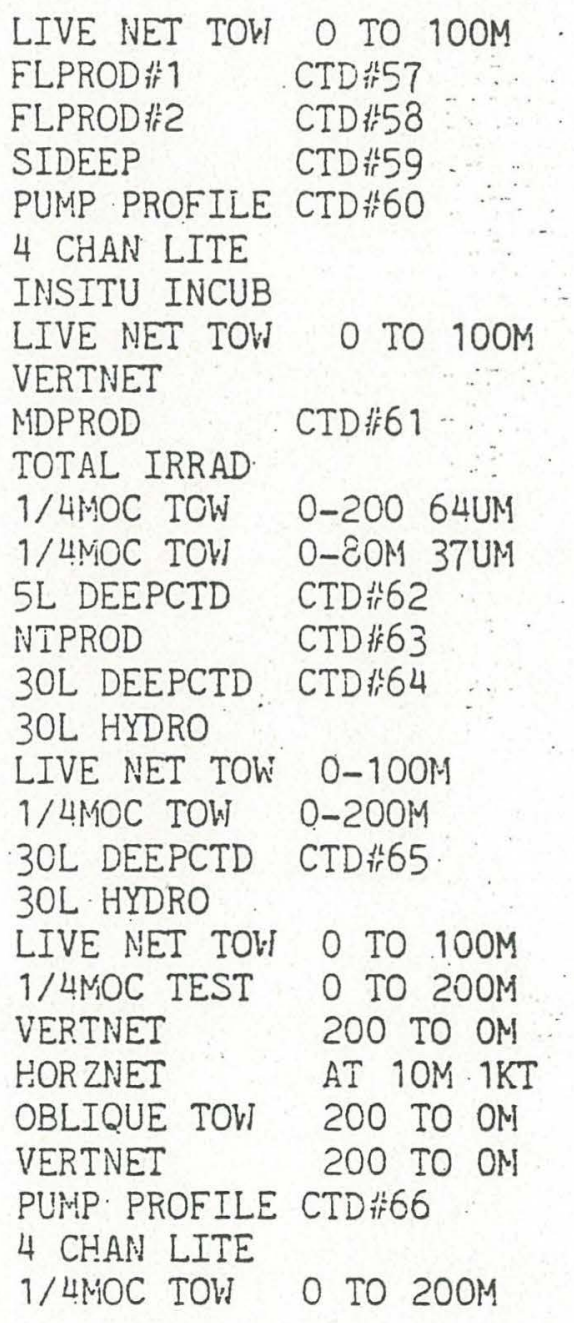

ITEMS THIS PAGE 30 ITEMS THIS REPORT 150 
TABLE 1 (continued) KN-093 OPERATION LCG

$05 / 05 / 82$

OP. NO. TIME STA LOCATION LAT. LONG. INVSTGR DESCRIPT COMMENT

\begin{tabular}{|c|c|c|c|c|c|c|c|c|}
\hline KN0503.08 & 1413 & 16 & RING CTR & 3855.471 & 39.6 & FRYXELL & $1 / 4 \mathrm{MOC}$ TOW & O TO $90 \mathrm{M}$ \\
\hline KNO503.09 & 1500 & 16 & RING CTR & 3857.471 & 40.0 & BROWN & 5L DEEPCTD & CTD俶 67 \\
\hline KNO503.10 & 2110 & 16 & RING CTR & 3857.871 & 39.6 & COWLES & 3OL HYDRO & $24 \mathrm{M}$ \\
\hline KN0503. 12 & 2129 & 16 & RING CTR & 3857.971 & 39.5 & COKLES & LIVE NET TOW & 0 TO $100 M$ \\
\hline KNO503.12 & 2200 & 16 & RING CTR & 3858.371 & 39.2 & MCCARTHY & NTPROD & CTD作68 \& \#69 \\
\hline 1110504.01 & 0500 & 16 & RING CTR & 3857.371 & 39.8 & MCCARTHY & FLPROD & CTD\#70 \\
\hline KN0504.02 & 0540 & 16 & RING CTR & 3857.371 & 39.8 & MCCARTHY & FLPROD非2 & CTD非1 1 \\
\hline N0504.03 & 0637 & 16 & RING CTR & 3857.671 & 40.0 & BRZEZINSKI & SIDEEP & CTD非72 \\
\hline KNO504.04 & 0800 & 16 & RING CTR & $38 \quad 57.7 \quad 71$ & 39.5 & FRYXELL & VERTNET & 200-0M 33UM \\
\hline KNO504.05 & 0900 & 16 & RING CTR & 3857.771 & 39.5 & ROMAN & INSITU INCUB & $0-36 M$ \\
\hline 04.06 & 1000 & 16 & RING CTR & 3857.771 & 39 & COWLES & LIVE NET TOW & \\
\hline 04.07 & 1030 & 16 & RING CTR & 3857.771 & 39 & COWLES & $1 / 4 M O C$ TOW & 200 TO OM \\
\hline 4.08 & 1200 & 16 & RING CTR & 3857.671 & 39 & MCCARTHY & MDPROD 茯 1 & CTD 1773 \\
\hline .09 & 130 & 16 & RING CTR & 3857.571 & 39 & MCCARTHY & MDPROD作2 & CTD佔 74 \\
\hline 4.10 & 1400 & 16 & RING CTR & 3857.971 & 39.6 & KESTER & 30L DEEPCTD & CTD 174 \\
\hline .11 & 1910 & 16 & RING CTR & 3857.571 & 40.0 & MCCARTHY & CTD TO $150 M$ & CTD非6 \\
\hline 12 & 2050 & 16 & RING CTR & 3857.571 & 40.0 & COVLES & LIVE NET TOH & O TO $100 \mathrm{MI}$ \\
\hline 04.13 & 2115 & 16 & RING CTR & 3857.471 & 39.6 & MCCARTHY & CTD TO $150 M$ & CTD非 77 \\
\hline .01 & 0510 & 17 & SLOPE & 3920.170 & 37.8 & BROWN & 5L DEEPCTD & CTD作78 \\
\hline .02 & 0750 & 17 & SLOPE & 3920.370 & 38 & RONAN & LIVE NET TOW & O TO 200M-1 \\
\hline 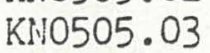 & 083 & 17 & SLOPE & 3921.170 & 38 & ROMAN & INSITU INCUB & \\
\hline 04 & 090 & 17 & SLOPE & 270 & 38.2 & YENTSCH & PUNIP PROFILE & CTD \\
\hline & 103 & 17 & SLOPE & 270 & 40 & YENTSCH & 4 CHAN LITE & \\
\hline & 11 & 17 & SLOPE & 3920 & 41 & YENTSCH & TOTAL IRRAD & \\
\hline & 12 & 17 & SLOPE & 3920.170 & 39. & BROWN & 3OL DEEPCTD & CTI \\
\hline 10 & 14 & 17 & SLOPE & 3921.970 & 40 . & COPLEY & LIVE NET TOW & \\
\hline & 15 & 13 & GULF STI & $3728.6 \quad 69$ & 50.8 & FRYXELL & LIVE NET TOW & TO OM \\
\hline & 18 & 8 & RADIAL & 3903.871 & 33.8 & FRYXELL & HOR ZNET & 35 UM \\
\hline & 103 & 9 & RING CT & $\begin{array}{lll}38 & 59.7 & 71\end{array}$ & 31.4 & YENTSCH & 4 CHAN LITE & \\
\hline & & y & RING C? & 871 & & RONAAN & $3 / 4 M$ NET TOW & 200 TO OH \\
\hline
\end{tabular}

ITENS THIS PAGE 30 ITEMS THIS REPORT 180 
$05 / 05 / 82$

PAGE \#. 1

\begin{tabular}{|c|c|c|c|c|c|c|c|c|}
\hline XBT \# & TIME-GHT & $D A / M O$ & LATITUDE & LONGITUDE & BUCKET & T. $z-15$ & $z-10$ & $z-06$ \\
\hline 001 & 0412 & $19 / 04$ & $3946.2 \mathrm{~N}$ & $6952.9 w$ & 07.2 & & .225 & 412 \\
\hline 002 & 0521 & $20 / 04$ & $3935.6 \mathrm{~N}$ & $6944.7 \mathrm{~W}$ & 08.4 & & 230 & 460 \\
\hline 003 & 0708 & $20 / 04$ & $3920.2 \mathrm{~N}$ & $6935.4 \mathrm{~W}$ & 07.5 & & 235 & 460 \\
\hline 004 & 2304 & $21 / 04$ & $39 \quad 18.7 N$ & $6959.7 \mathrm{H}$ & 10.4 & & 240 & 500 \\
\hline 005 & 0005 & $22 / 04$ & $3924.8 N$ & $70 \quad 13.1 \mathrm{ij}$ & 10.0 & & 240 & 480 \\
\hline 006 & 0100 & $22 / 04$ & $3929.7 \mathrm{~N}$ & $7023.7 W$ & 09.5 & & 237 & 480 \\
\hline 007 & 0200 & $22 / 04$ & $3926.9 \mathrm{~N}$ & $7034.1 \mathrm{~W}$ & 11.6 & & 290 & 485 \\
\hline 008 & 0300 & $22 / 04$ & $\begin{array}{ll}39 & 17.9 \mathrm{~N}\end{array}$ & $7044.0 W$ & 12.5 & & 350 & 580 \\
\hline 009 & 0400 & $22 / 04$ & $3911.9 \mathrm{~N}$ & $7053.7 \mathrm{~W}$ & 13.7 & & 420 & 570 \\
\hline 010 & 0500 & $22 / 04$ & $3904.9 \mathrm{~N}$ & $7002.7 \mathrm{~W}$ & 15.2 & 255 & 470 & 710 \\
\hline 011 & 0607 & $22 / 04$ & $3857.0 \mathrm{~N}$ & $7114.0 \mathrm{~W}$ & 15.6 & 408 & 551 & 780 \\
\hline 012 & 0702 & $22 / 04$ & $3850.7 N$ & $7122.6 \mathrm{~W}$ & 15.4 & 430 & 575 & 790 \\
\hline 13 & 0803 & $22 / 04$ & $3857.6 \mathrm{~N}$ & $7131.9 \mathrm{~W}$ & 15.7 & 432 & 565 & 755 \\
\hline 14 & 0859 & $22 / 04$ & $3857.6 N^{\prime}$ & $7119.8 \mathrm{~W}$ & 15.4 & 442 & 565 & 790 \\
\hline 15 & 2000 & $23 / 04$ & $3855.7 \mathrm{~N}$ & $7140.8 \mathrm{~W}$ & 15.0 & 350 & 570 & 750 \\
\hline 16 & 2100 & $23 / 04$ & $3906.2 \mathrm{~N}$ & $7137.5 \mathrm{~W}$ & 15.5 & 380 & 530 & 750 \\
\hline 17 & 2200 & $23 / 04$ & $3916.6 \mathrm{~N}$ & $7134.1 \mathrm{~W}$ & 15.3 & 325 & 500 & \\
\hline 018 & 2300 & $23 / 04$ & $3927.6 \mathrm{~N}$ & $7131.7 \mathrm{~W}$ & 13.0 & & 370 & 560 \\
\hline 019 & 0400 & $24 / 04$ & $3927.6 \mathrm{~N}$ & $7131.8 \mathrm{w}$ & & & 340 & 550 \\
\hline 020 & 0700 & $25 / 04$ & $38.49 .8 \mathrm{~N}$ & $7138.6 \mathrm{H}$ & 13.0 & 420 & 550 & 750 \\
\hline 021 & 0800 & $25 / 04$ & $3855.5 \mathrm{~N}$ & $7134.1 \mathrm{~W}$ & 14.4 & 420 & 555 & 750 \\
\hline 022 & 0830 & $25 / 04$ & $3859.9 \mathrm{~N}$ & $7129.8 \mathrm{~W}$ & 14.7 & 430 & 565 & 740 \\
\hline 023 & 0952 & $25 / 04$ & $3900.4 \mathrm{~N}$ & $7143.3 w$ & 15.0 & 320 & 532 & 740 \\
\hline 024 & 0117 & $27 / 04$ & $3856.9 \mathrm{~N}$ & $7104.4 \mathrm{~W}$ & 15.8 & 243 & 438 & 640 \\
\hline 025 & 0200 & $27 / 04$ & $3856.1 \mathrm{~N}$ & $7055.8 \mathrm{~W}$ & 13.5 & 170 & 400 & 618 \\
\hline 026 & 0312 & $27 / 04$ & $3849.8 \mathrm{~N}$ & $7056.4 \mathrm{~W}$ & 13.3 & & 365 & 590 \\
\hline 027 & 0800 & $27 / 04$ & $3844.2 N$ & $7103.3 \mathrm{~W}$ & 13.2 & & 380 & 650 \\
\hline 028 & 1432 & $28 / 04$ & $3853.0 \mathrm{~N}$ & $7139.0 \mathrm{~W}$ & 15.7 & 410 & 535 & 750 \\
\hline 029 & 1437 & $28 / 04$ & $3853.0 \mathrm{~N}$ & $7139.0 \mathrm{~W}$ & 15.5 & 410 & 540 & 750 \\
\hline 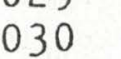 & 1447 & $28 / 04$ & $3853.0 \mathrm{~N}$ & $7139.0 \%$ & 15.5 & 405 & 530 & 740 \\
\hline L & IS PAGE & 30 & S THIS & 30 & & & & \\
\hline
\end{tabular}




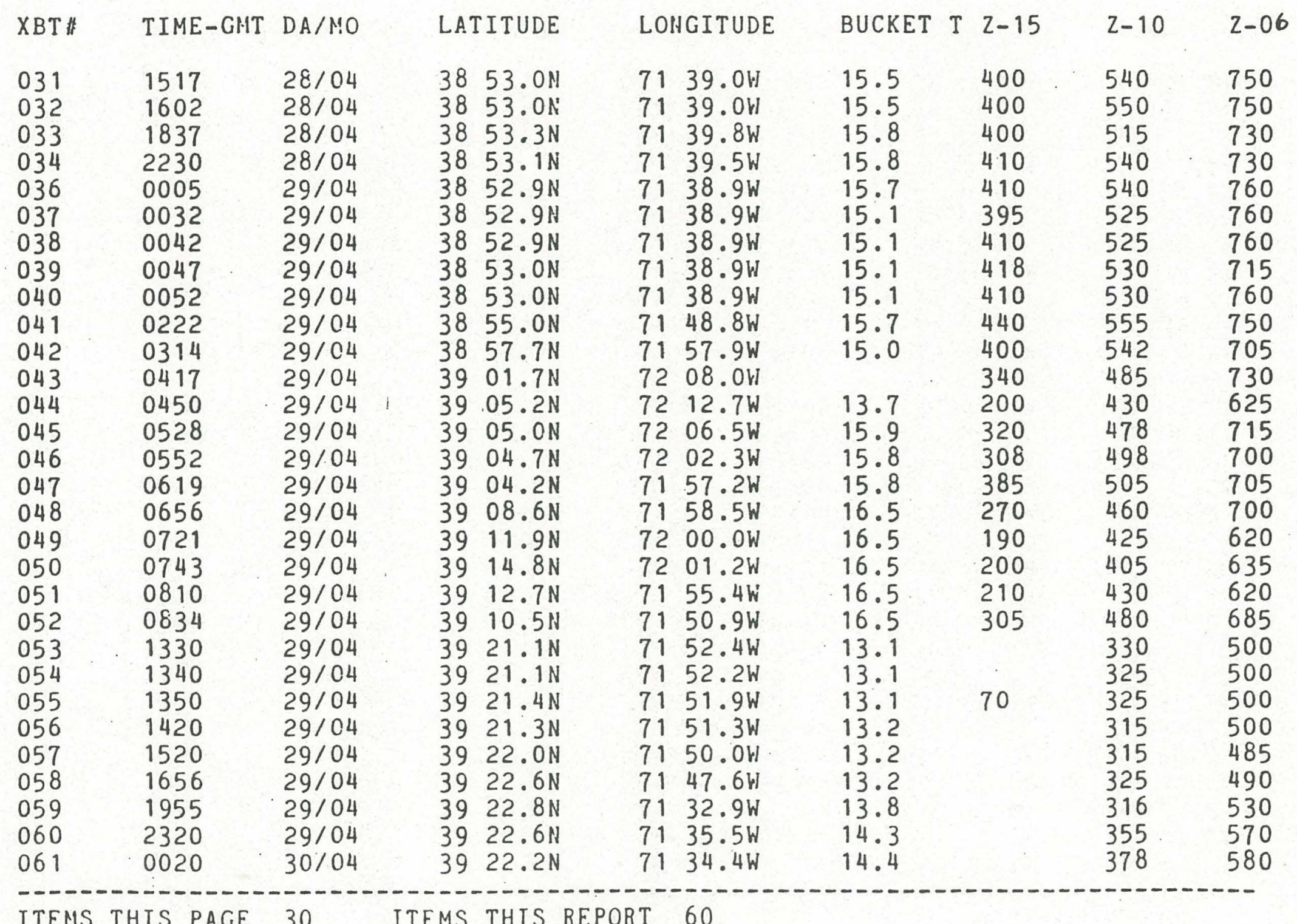




\begin{tabular}{|c|c|c|c|c|c|c|c|c|}
\hline XBT & TIME-GMT & $\mathrm{DA} / \mathrm{MO}$ & LATITUDE & LONGITUDE & BUCKET T & $z-15$ & $z-10$ & $z-06$ \\
\hline 062 & 0120 & $30 / 04$ & $3921.5 \mathrm{~N}$ & $7133.4 \mathrm{~W}$ & 13.0 & & 395 & 590 \\
\hline 063 & 0210 & $30 / 04$ & $3920.4 \mathrm{~N}$ & $7133.2 W$ & 14.4 & & 388 & 590 \\
\hline 064 & & $30 / 04$ & & & & & 336 & 575 \\
\hline 065 & 1030 & $30 / 04$ & $38 \quad 18.6 \mathrm{~N}$ & $7039.4 k$ & 12.6 & & 215 & 4.10 \\
\hline 066 & 1226 & $30 / 04$ & $3800.8 \mathrm{~N}$ & $7026.4 \mathrm{~W}$ & 17.6 & 20 & 193 & 415 \\
\hline 067 & 1310 & $30 / 04$ & $3755.7 N$ & $70 \quad 22.2 W$ & 21.5 & 5 & 200 & 425 \\
\hline 068 & 1405 & $30 / 04$ & $3747.6 \mathrm{~N}$ & $70 \quad 15.9 \mathrm{~W}$ & 20.5 & 25 & 200 & 380 \\
\hline 069 & 1455 & $30 / 04$ & $3739.8 \mathrm{~N}$ & $70 \quad 10.2 \mathrm{~W}$ & 19.5 & 43 & 260 & 440 \\
\hline 070 & 1605 & $30 / 04$ & $3731.5 \mathrm{~N}$ & $7004.1 \mathrm{H}$ & 23.5 & 122 & 346 & 541 \\
\hline 071 & 1658 & $30 / 04$ & $3725.2 \mathrm{~N}$ & $6959.9 w$ & 24.5 & 270 & & \\
\hline 072 & 1728 & $30 / 04$ & $3721.0 \mathrm{~N}$ & $6957.5 \mathrm{~W}$ & 23.4 & 325 & 547 & 752 \\
\hline 073 & 0200 & $02 / 05$ & $3720.2 \mathrm{~N}$ & $6937.8 w$ & 23.4 & 540 & 707 & \\
\hline 074 & 0400 & $02 / 05$ & $3701.6 \mathrm{~N}$ & $6932.2 w$ & 18.9 & 627 & & \\
\hline 075 & 0603 & $02 / 05$ & $3642.2 \mathrm{~N}$ & $6925.5 \mathrm{~W}$ & 21.0 & 645 & & \\
\hline 076 & 0700 & $02 / 05$ & $3646.7 \mathrm{~N}$ & $6937.9 \mathrm{~W}$ & 19.0 & 605 & & \\
\hline 077 & 0813 & $02 / 05$ & $3656.1 \mathrm{~N}$ & $6952.1 \mathrm{~W}$ & 22.5 & 617 & & \\
\hline 077 & 0813 & $02 / 05$ & $3656.1 \mathrm{~N}$ & $6952.1 \mathrm{~W}$ & 22.5 & 617 & & \\
\hline 078 & 0900 & $02 / 05$ & $3656.4 \mathrm{~N}$ & $7001.4 \mathrm{~W}$ & 22.5 & 570 & 750 & \\
\hline 079 & 1003 & $02 / 05$ & $3703.0 \mathrm{~N}$ & $7013.4 W^{\prime}$ & 25.0 & 490 & 650 & \\
\hline 080 & 1100 & $02 / 05$ & $3708.4 \mathrm{~N}$ & $7022.1 \mathrm{~W}$ & 25.0 & 355 & 485 & 700 \\
\hline 081 & 1810 & $02 / 05$ & $3533.3 N$ & $7115.8 \mathrm{~W}$ & 16.7 & 8 & 170 & 400 \\
\hline 083 & 2005 & $02 / 05$ & $3711.7 \mathrm{~N}$ & $7127.7 \mathrm{~W}$ & 15.5 & & 270 & 505 \\
\hline 084 & 2200 & $02 / 05$ & $3832.2 N$ & $7134.4 \mathrm{k}_{\mathrm{i}}$ & 16.7 & 300 & 450 & 670 \\
\hline 085 & 000.0 & $03 / 05$ & $3854.4 \mathrm{~N}$ & $7139.8 \mathrm{~W}$ & 16.7 & 455 & 575 & \\
\hline 086 & 0200 & $05 / 05$ & $3859.3 N$ & $7132.4 \mathrm{~W}$ & 16.6 & 365 & 507 & 718 \\
\hline 087 & 0300 & $05 / 05$ & $39.03 .6 \mathrm{~N}$ & $7121.4 \mathrm{~W}$ & 16.7 & 240 & 420 & 58 \\
\hline 088 & 0400 & $05 / 05$ & $3908.3 N$ & $7108.7 \mathrm{~W}$ & 9.5 & & 30.3 & 450 \\
\hline 089 & 0518 & $05 / 05$ & $3915.1 \mathrm{~N}$ & $7052.5 \mathrm{~W}$ & 10.8 & & 200 & 370 \\
\hline 090 & 0610 & $05 / 05$ & $3919.5 \mathrm{~N}$ & $7041.6 \mathrm{~W}$ & 11.7 & & $227^{\circ}$ & 407 \\
\hline
\end{tabular}


CRUISE REPORT

OCEANUS CRUISE NO. $118,4 / 18 / 82-5 / 3 / 82$

Peter $\mathrm{H}$. Wiebe

Woods Hole Oceanographic Institution

Woods Hole, Massachusetts

\section{Contents}

I. Cruise Narrative

II. Individual Reports

III. Scientific Observation Lọ

IV. XBT Digitizing Log 
OCEANUS Cruise No. $118,4 / 18 / 82-5 / 3 / 82$

Peter H. Wiebe

Woods Hole Oceanographic Institution

R/V OCEANUS left port on 18 April as one of four ships participating in the first cruise of a time-series study of a Gulf Stream warm core ring. The other ships in this coordinated study were R/V ENDEAVOR, R/V KNORR and the R/V ALBATROSS IV. In addition, a NASA P-3 aircraft made two overflights of the ring while the research vessels were in the ring environs. The overali objective of the scientific research aboard the OCEANUS was to map the ring's. horizontal and vertical distributions of zooplankton and microne'ton, and to study the vertical distribution of size fractionated particulate matter, chlorophyll a, radon, radium 228 and radium 226 .

As a result of time required to make major modifications to the traw 1 winch sheave train, to install the ATLANTIS II's A-frame on the OCEANUS, to construct a starboard support for the crane, to complete construction of two new towing blocks and to do a strength test on the trawl wire train, we left port three days late. These modifications were required to accommodate our large trawls (the MOCNESS-20 and the double MOCNESS-1), the large volume pumping system (LVFS), and the six-shooter (an STD/30 liter Rosette/radium pumping system).

We steamed from Woods Hole, stopped for an hour in Vineyard Sound for testing the launch and recovery procedure for the six-shooter, and then steamed out to a Slope Water station $\left(39^{\circ} 20^{\prime} \mathrm{N} ; 69^{\circ} 30^{\prime} \mathrm{W}\right)$ which was $\sim 130$ nautical miles northwest of ring $82 \mathrm{D}$ and $\sim 80$ miles northeast of ring $82 \mathrm{~B}$. This location was chosen for our first station to permit the ENDEAVOR, which left port 24 hours ahead of us, to complete an XBT survey designed to assess the feasibility of using ring $82 \mathrm{D}$ as opposed to $82 \mathrm{~B}$ as the time-series study site and to exercise our gear in a relatively low current regime.

Based on the ENDEAVOR results, $82 \mathrm{~B}$ was selected as the study ring. After completing work at the Slope Water station on 21 April, we steamed to the center of this ring, and over a two-day period, completed a major station which included a day/night pair of tows with the double MOCNESS-1 and the MUCNESS-20, an 8-sample LVFS series from the surface to 700 meters, an i1-bottle chlorophy 11 cast to 100 meters, a shallow and deep radon cast, and a six-shooter lowering. Between the 24th and 26th of April, two stations ( 3 and 4 ) in the high velocity region of the southwest portion of the ring followed the center station. We intended to complete another set of observations in the ring center (station 5) on the 27th prior to the first joint ship experiment, but a storm with winds up to 50 knots forced us to curtail the net towing and some of the LVFS work. This storm also caused the cancellation of much of the work we intended to do during the joint ship experiment in the ring center on the 28th. Work was limited to hydro-wire casts with 5- and 3U-liter bottles for analysis of chlorophyll and radon, respectively. The weather improved enough on the 29 th to permit the second joint ship experiment 
to be carried out in the high velocity region of the northest sector of the ring. At this station (no. 6), we did a shallow radon cast, a chlorophyll cast, a day/night pair of MOCNESS-1 tows, and a shallow LVFS cast in the vicinity of the Loran drifter/plank-on-edge buoy combination set out early on the 2yth by the scientific party aboard the R/V ENDEAVOR.

The OCEANUS returned to the ring center on the 30 th to further characterize the biological and chemical characteristics of the ring core (station no. 7). After this work was completed on May 1, we steamed to a Slope Water location to the northeast of the ring center $\left(39^{\circ} 30^{\prime} \mathrm{N} ; 71^{\circ} 00^{\prime} \mathrm{W}\right.$ ) for our final station (no. 8) where a nearly complete set of observations was obtained; only a MUCNESS-20 daytime tow was omitted because of time lost $(\sim 5$ hrs.) when the trawl wire unraveled on the drum as the trawl was being brought on board on the previous night tow

A summary of the placement of the net tows and radon, chlorc, hyll, LVFS, and six-shooter casts are graphically presented in Figure 1. Table 1 gives the ring center positions used to calculate distances of individual observations from the ring center. A summary of events is given in Section 3; XBT data are given in Section 4. Individual principal investigators reports follow.

In spite of the loss of two days of ship time caused by the delay in sailing and that lost to bad weather, and in spite of a variety of electronics problems afflicting the MUCNESS and the six-shooter, this cruise can be characterized as quite successful because of a full complement of data obtained at the various locations around the ring. Our success is in no small way due to the full and friendly cooperation of the officers and crew of the UCEAIVUS. Their hard work and assistance is greatly appreciated.

Table 1. Ring Center Positions

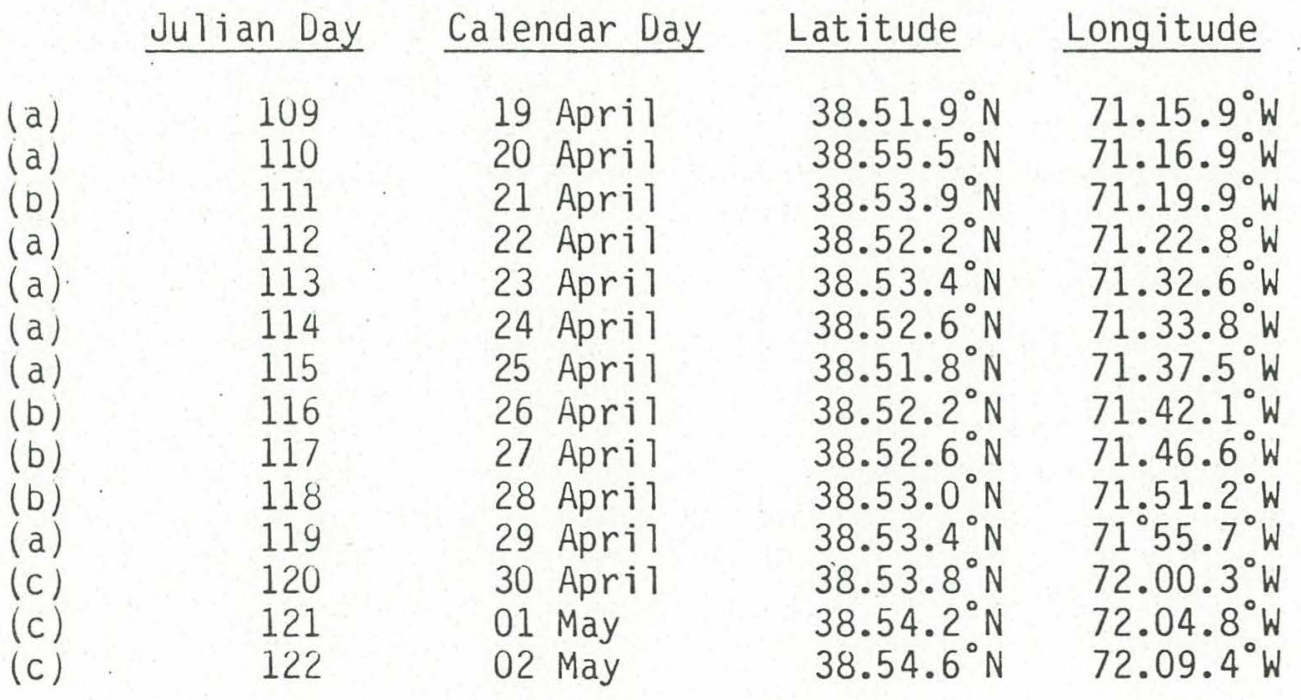

(a) Uata from Utis Brown, University of Miami

(b) Interpolated

(c) Extrapolated 
Figure 1.

WARM-CORE RINGS TIME SERIES

OCEANUS 118
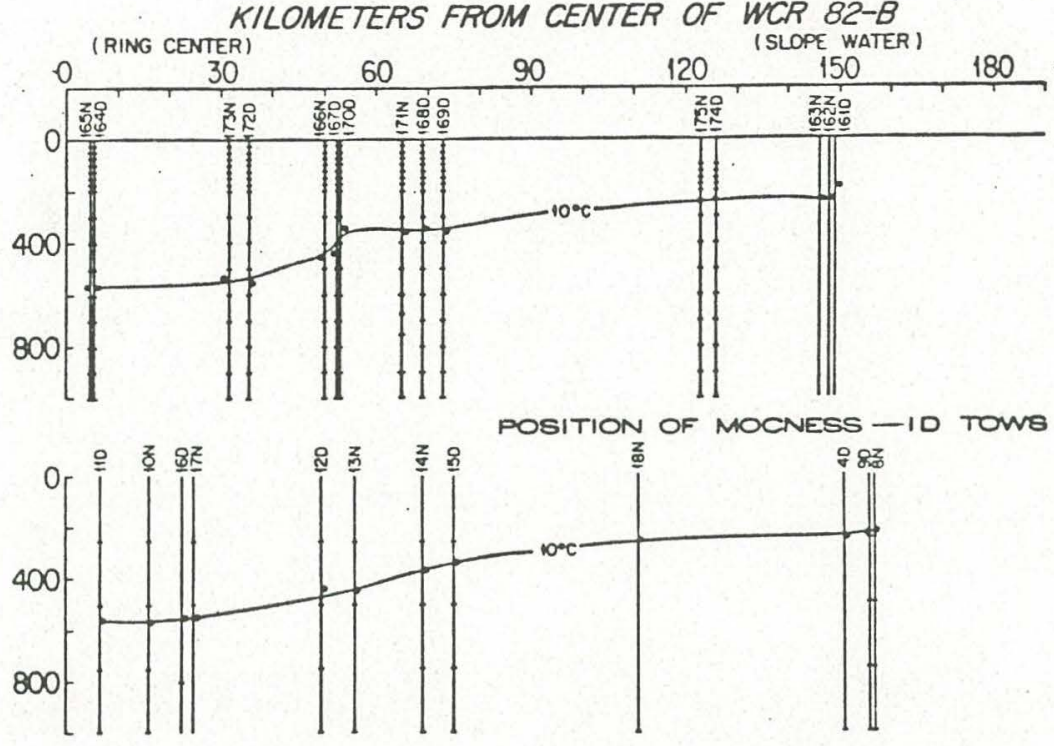

POSITION OF MOCNESS - 20 TOWE
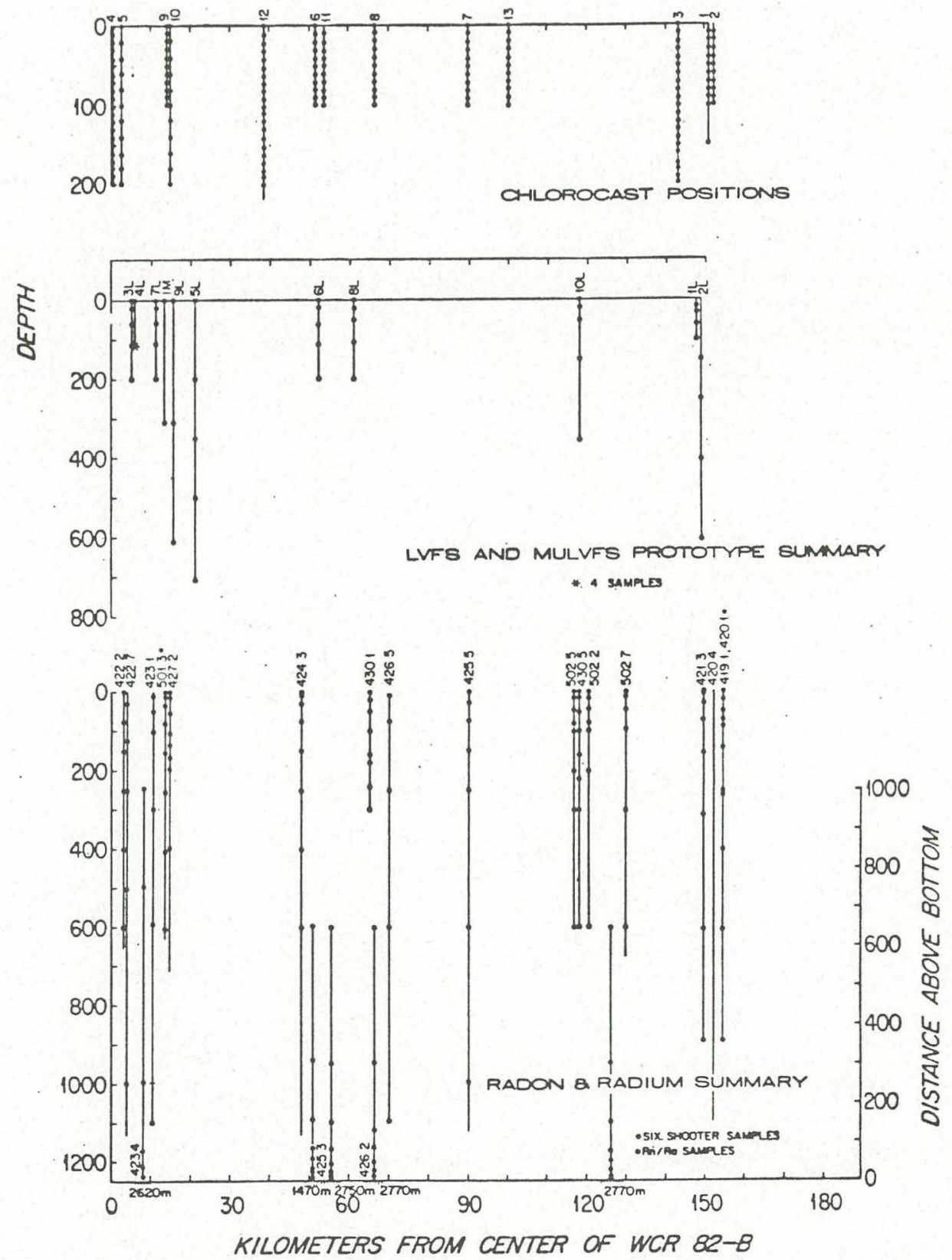
II. INDIVIDUAL REPURTS

\title{
PARTICULATE MATTER STUDIES
}

\author{
Principal Investigator: James K. B. Bishop \\ Cruise Participants: Robert Sherrel1, Maureen Conte and James K. B. Bishop \\ Lamont-Doherty Geological Observatory
}

Tne objectives of large volume in situ filtration studies during OCEANUS cruise 118 were fourfold:

1) to obtain size fractionated particulate matter samples from various environments in warm core ring systems so that factors governing partulate matter chemistry, reactivity, and sedimentation rates may be understood.

2) to test the deployment of sediment traps on the LVFS.

3) to test new filter holder and modified prototype designs for the Multiple Unit Large Volume In Situ Filtration System (MULVFS).

4) to test Gelman microquartz filter material as a substitute for Mead $935-3 \mathrm{~J}$ glass fiber filters.

All these objectives were accomplished.

The deployments of the LVFS and MULVFS prototype were entirely successful, and 40 samples were collected from Slope Water, the high velocity region, and ring center stations from a depth range of 15 to 700 meters (Table 1). Not one component of either system malfunctioned in spite of occasional high sea state. The OCEANUS was an excellent sampling platform. Traps which were deployed on the LVFS on three casts (Table 2) returned material for gravimetric and chemical analysis. The new filter holder design appeared to De an improvement over the LVFS filter holders. The Gelman filters, although snowing slightly slower filtration rates than the Mead filters, performed we11. A decision on the suitability of the Gelman material will have to await tne results of chemical analyses at Lamont-Doherty Geological Observatory.

Two additional experiments undertaken on the R/V ENDEAVOR were to deploy a 1 -meter transmissometer on the CTD/Rosette to gain a high resolution view of the spatial variations of particulate matter and also to deploy Martin- and Knauer-style sediment traps on the Loran $C$ navigated drifter deployed in the ring center and high velocity region. The transmissometer failed on the first lowering in the Slope Water, and attempts to repair the instrument were suspended as the instrument was under warranty. The sediment traps were deployed on the drifter in the ring center at 113 meters, and three of four traps were recovered. Traps could not be redeployed in the HVR due to excessive sea state conditions. The three samples were allowed to drain under gravity and were stored under refrigeration.

Continuous $12 \mathrm{KHz}$ echo sounder recordings were made during the cruise except during deep radon casts (Figure 1 ). These records profiled the scattering layers to $75 \mathrm{Um}$, although the upper $50 \mathrm{~m}$ were frequently obscured. 


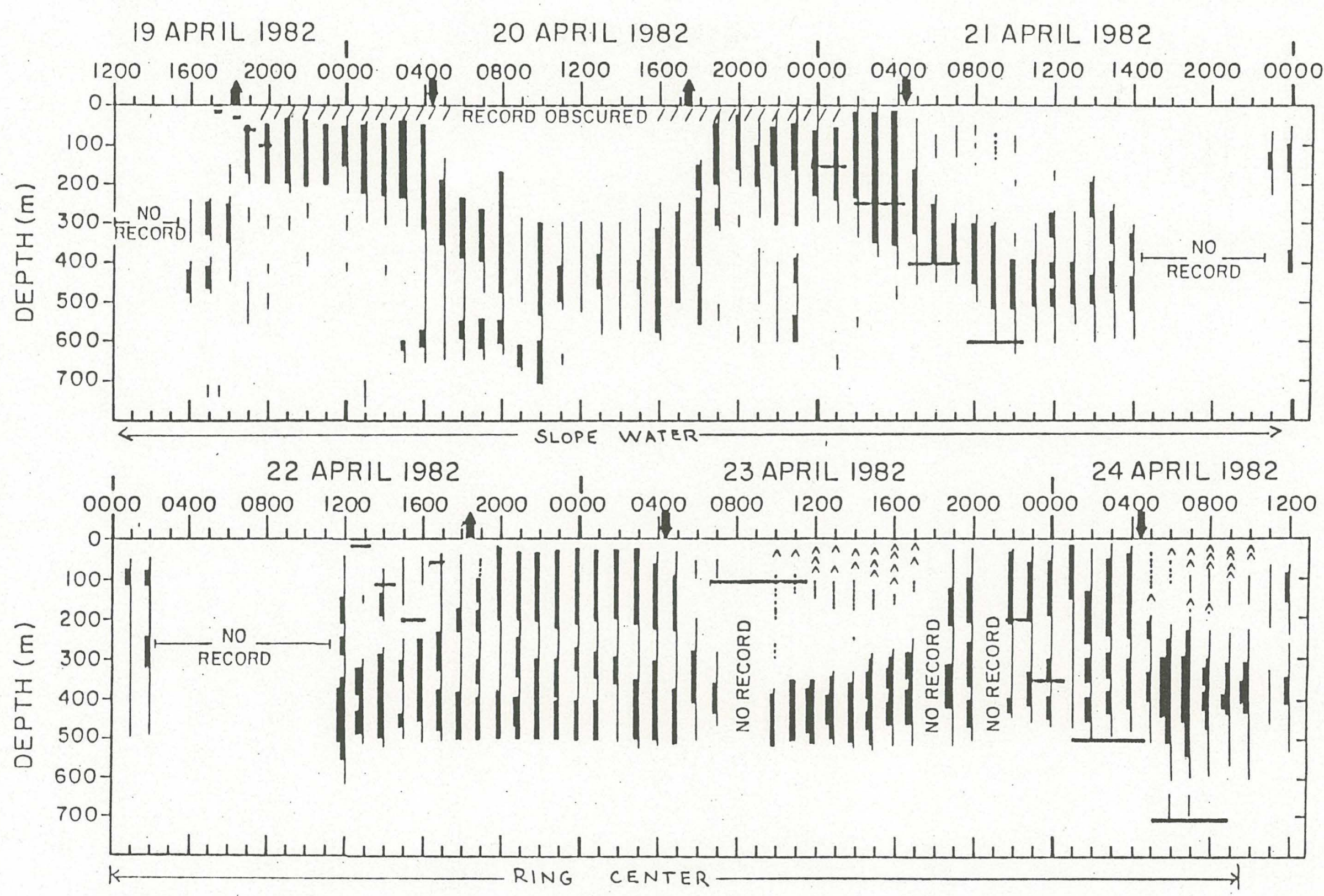

Fisure 1. Hourly summary of maxtmum scattering depths from $12 \mathrm{KHz}$ echo sounder recordings. 


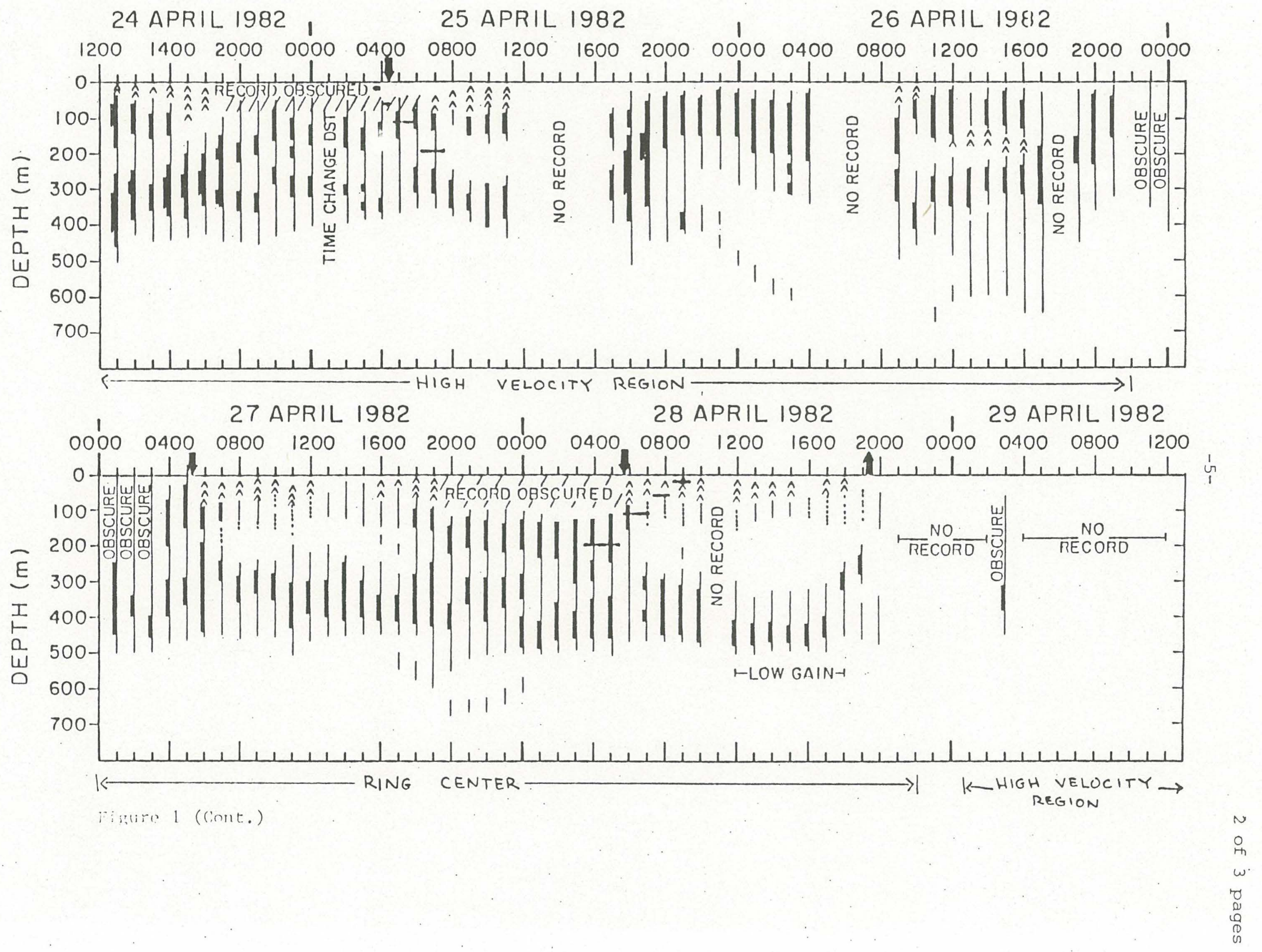




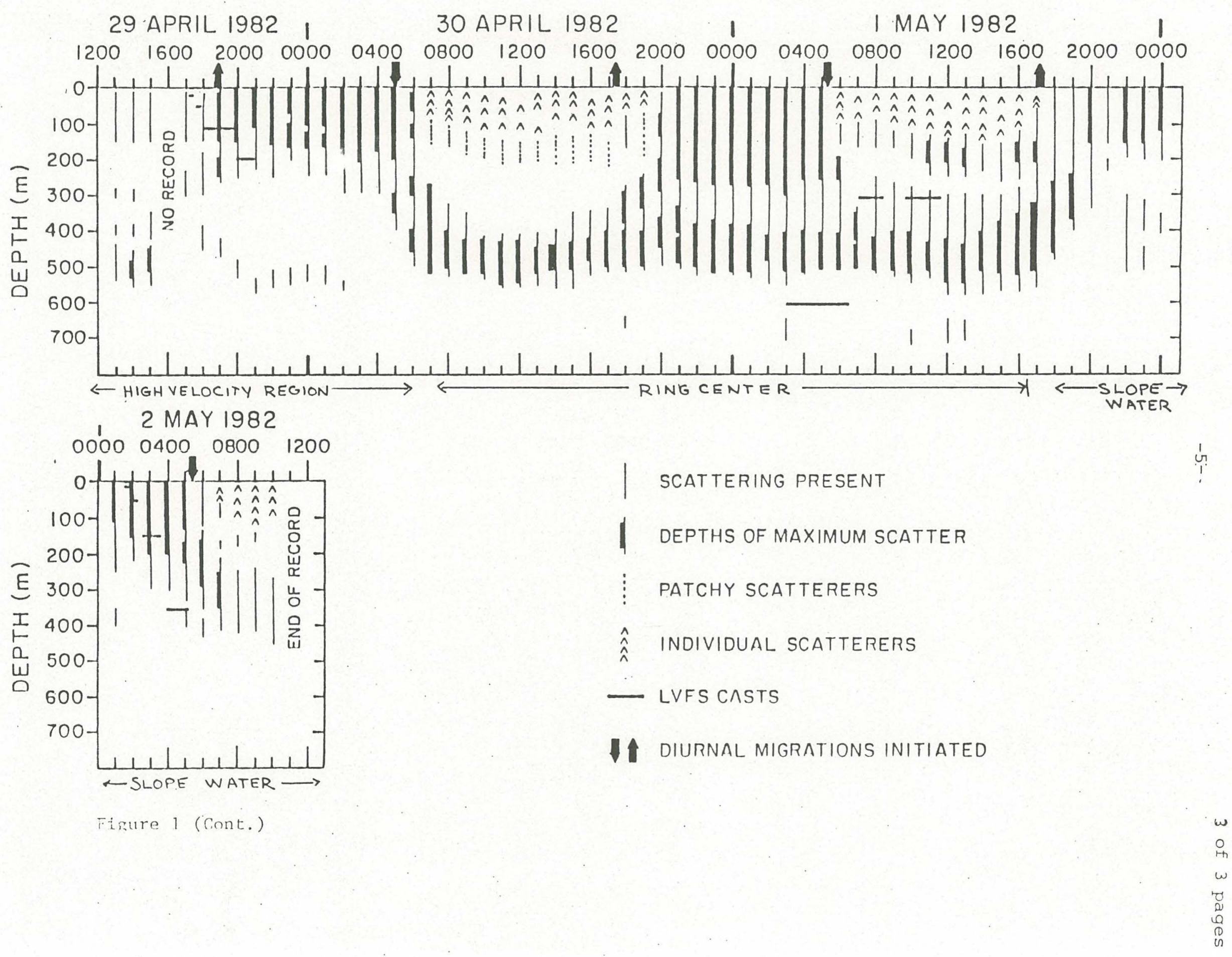


The majority of the scattering was confined to the upper $500 \mathrm{~m}$ with some scattering layers present at $600 \mathrm{~m}$ or greater in all three regions. The Slope water was dominated by scatters showing strong diurnal migration patterns. maximum scattering was concentrated between 250 and $550 \mathrm{~m}$ during mid-day and in the upper 3 uum at night. In contrast, the ring center waters exhibited a strong, non-migrating scattering layer betweeen 350 and $500 \mathrm{~m}$ in adddition to diurnal migrators. The night-time water column contained many scattering layers throughout the 0 to $500 \mathrm{~m}$ range. The ring center also exhibited patches between 100 and $200 \mathrm{~m}$ and a greater number of individual signals during the day. The nigh velocity zone was distinct from either the Slope or ring center waters. Maximum scattering was generally confined above $400 \mathrm{~m}$ and, unlike the slope Water and ring core, strong scattering layers were present in the upper zoum during the day.

Bishop, et al.

Table 1. LVFS and MULVFS Cast Summary, 2 May 1982

vate Uay Cast Sta Time Local Depths Mean Position Dist Samp from

R.C.

\begin{tabular}{|c|c|c|c|c|c|c|c|c|c|c|c|}
\hline $4 / 19 / 82$ & luy & $1 \mathrm{~L}$ & 1 & +5 & 1720 & 2103 & $\begin{array}{l}15 \\
60\end{array}$ & $\begin{array}{r}30 \\
100\end{array}$ & $39.13 .0^{\circ} \mathrm{N}$ & $69.36 .8^{\circ} \mathrm{W}$ & 79.7 \\
\hline $4 / 2 U / 82$ & 110 & $2 \mathrm{~L}$ & 1 & +5 & 2355 & 1030 & $\begin{array}{l}150 \\
405\end{array}$ & $\begin{array}{l}250 \\
605\end{array}$ & $39.08 .6^{\circ} \mathrm{N}$ & $69.38 .6^{\circ} \mathrm{W}$ & 80.2 \\
\hline $4 / 22 / 82$ & 112 & $3 \mathrm{~L}$ & 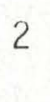 & +5 & 1218 & 1707 & $\begin{array}{r}20 \\
112\end{array}$ & $\begin{array}{r}60 \\
200\end{array}$ & $38.51 .1^{\circ} \mathrm{N}$ & $71.20 .0^{\circ} \mathrm{W}$ & 2.8 \\
\hline $4 / 23 / 82$ & 13 & $4 \mathrm{~L}$ & 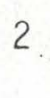 & +5 & $0647^{\circ}$ & 1137 & $\begin{array}{l}112 \\
112\end{array}$ & $\begin{array}{l}112 \\
112\end{array}$ & $38.49 .9^{\circ} \mathrm{N}$ & $71.33 .2^{\circ} \mathrm{W}$ & 3.2 \\
\hline $4 / 23 / 82$ & 13 & $5 \mathrm{~L}$ & , & +5 & 2303 & 0856 & $\begin{array}{l}200 \\
500\end{array}$ & $\begin{array}{l}350 \\
707\end{array}$ & $38.43 .6^{\circ} \mathrm{N}$ & $71.42 .6^{\circ} \mathrm{W}$ & 11.3 \\
\hline $4 / 25 / 82$ & 115 & $6 \mathrm{~L}$ & & 4 & 0330 & 0744 & $\begin{array}{r}21 \\
112\end{array}$ & $\begin{array}{r}62 \\
200\end{array}$ & $38.34 .9^{\circ} \mathrm{N}$ & $72.07 .2^{\circ} \mathrm{W}$ & 28.5 \\
\hline $4 / 28 / 82$ & 118 & $7 \mathrm{~L}$ & 5 & +4 & 0332 & 0914 & $\begin{array}{r}20 \\
112\end{array}$ & $\begin{array}{r}60 \\
200\end{array}$ & $38.49 .3^{\circ} \mathrm{N}$ & $71.43 .9^{\circ} \mathrm{W}$ & 5.4 \\
\hline $4 / 2 y / 92$ & 114 & $8 \mathrm{~L}$ & 6 & +4 & 1708 & 2152 & $\begin{array}{r}21 \\
110\end{array}$ & $\begin{array}{r}50 \\
200\end{array}$ & $39.23 .1^{\circ} \mathrm{N}$ & $71.37 .3^{\circ} \mathrm{W}$ & 32.9 \\
\hline $5 / 01 / 82$ & 121 & GL & 7 & +4 & 0258 & 0855 & $\begin{array}{r}310 \\
\text { bla }\end{array}$ & $\begin{array}{l}610 \\
\text { ank }\end{array}$ & $38.52 .6^{\circ} \mathrm{N}$ & $71.54 .5^{\circ} \mathrm{W}$ & 8.4. \\
\hline $5 / 01 / 82$ & & $1 \mathrm{~s}$ & & +4 & & 1134 & 308 & & $38.53 .7^{\circ} \mathrm{N}$ & $71.55 .7^{\circ} \mathrm{W}$ & 7.1 \\
\hline & & & 8 & +4 & 0147 & 050 & $\begin{array}{r}15 \\
150\end{array}$ & $\begin{array}{r}52 \\
355\end{array}$ & & & \\
\hline
\end{tabular}

Key: vay, Julian Vay; Cast, L=LVFS; M=MULVFS prototype; Time ${ }^{+n}$ hours earlier than GilT; Depths sampled, meters; Distance, distance from ring center in nautical miles based on satellite data. 
Bishop, et al.

Table 2. LVFS Sediment Trap Deployment Summary, 2 May 1982

\begin{tabular}{|c|c|c|c|c|c|c|c|}
\hline Uate & Uay & Cast & Sta & Time ( $\mathrm{L}$ & $=a 1)$ & Depth Range & Trap Numbers \\
\hline $4 / 28 / 82$ & 118 & $7 \mathrm{~L}$ & 5 & 0300 & 0930 & $20-200 \mathrm{~m}$ & 13,14 \\
\hline $4 / 29 / 82$ & 119 & $8 \mathrm{~L}$ & 0 & 1700 & 2210 & $20-200 m$ & 15,16 \\
\hline $5 / 01 / 82$ & 121 & $9 \mathrm{~L}$ & 7 & $+4 \quad 0200$ & 0855 & $310-610 m$ & 17,18 \\
\hline
\end{tabular}

Sediment traps were attached to filter holder No. 1 on LVFS.

\section{RADON AND RADIUM IN WARM CORE RINGS}

Principal Investigator: David R. Schink

Cruise Participants: Norman L. Guinasso, James Orr, and Patrick Setser Texas AaM University

Our purpose was to measure radium 226 , radium 228 , and radon 222 . The distribution of these isotopes should provide insight into horizontal and vertical mixing processes that affect the biology and chemistry of warm core rings.

we made three different kinds of casts to collect our samples.

Surface radon casts (SR) were made with 30 liter Niskin bottles on the hydrowire usually in the upper few hundred meters of the water column. Reversing theremometers were used to measure temperature; salinity and oxygen were measured on samples taken from these bottles. Radon was measured and counted aboard ship. Radium was extracted on treated manganese fiber and will be returned to our laboratory for analys is of radium 226 . Samples for nutrient determination were frozen for later analysis.

Ueep radon casts (DR) were taken with 8, 30-1iter Niskin bottles on the nyarowire. Bottles were positioned very near the bottom with the aid of a pinger. The bottom bottle typically was tripped about 5 meters off the Dottom. Uther bottles were generally within 300 meters of the bottom. salinities and oxygen were determined on samples taken from these casts. Samples for nutrient determination were frozen for later analysis.

six-shooter (SS) casts were taken to measure radium 228 . This device is an in situ pumping system which pumps six, 1000 liter samples through acrylic fiber filters treated with permanganate to permit the filters to adsorb radium. Un tinis cruise, this system was lowered attached to a 9040 STD. The STU data was aigitized and recorded for later analysis. Data on the salinity channel was somewhat noisy.

vur group also ran surface salinity samples for each XBT station and for each weatner observation. 
The table below gives a summary of our activities. SRa denotes a surface cast where tne radon samples were not run, but radium samples were taken. Day denotes the Julian Uay. Latitude and longitude are given in degrees.

Schink, et al.

Table 1.

\begin{tabular}{|c|c|c|c|c|c|}
\hline Uay & Cast & Est. Ring Center & Station Position & Dist. RC. & $(\mathrm{NM})$ \\
\hline IUY & 419.1 & $38.865^{\circ} \mathrm{N} ; 71.265^{\circ} \mathrm{W}$ & $39.140^{\circ} \mathrm{N} ; 69.521^{\circ} \mathrm{W}$ & 82.96 & SR \\
\hline $11 U$ & 420.1 & $38.925^{\circ} \mathrm{N} ; 71.282^{\circ} \mathrm{W}$ & $39.277^{\circ} \mathrm{N} ; 69.558^{\circ} \mathrm{W}$ & 82.97 & SS \\
\hline 110 & 420.4 & $38.925^{\circ} \mathrm{N} ; 71.282^{\circ} \mathrm{W}$ & $39.263^{\circ} \mathrm{N} ; 69.578^{\circ} \mathrm{W}$ & 81.88 & STD \\
\hline 111 & 421.3 & $38.898^{\circ} \mathrm{N} ; 71.332^{\circ} \mathrm{W}$ & $39.182^{\circ} \mathrm{N} ; 69.645^{\circ} \mathrm{W}$ & 80.42 & SRa \\
\hline 112 & 422.2 & $38.870^{\circ} \mathrm{N} ; 71.380^{\circ} \mathrm{W}$ & $38.840^{\circ} \mathrm{N} ; 71.385^{\circ} \mathrm{W}$ & 1.82 & SR \\
\hline 112 & 422.7 & $38.870^{\circ} \mathrm{N} ; 71.380^{\circ} \mathrm{W}$ & $38.907^{\circ} \mathrm{N} ; 71.388^{\circ} \mathrm{W}$ & C.23 & SS \\
\hline 113 & 423.1 & $38.890^{\circ} \mathrm{N} ; 71.543^{\circ} \mathrm{W}$ & $38.840^{\circ} \mathrm{N} ; 71.437^{\circ} \mathrm{W}$ & 5.82 & STD \\
\hline 113 & 423.4 & $38.890^{\circ} \mathrm{N} ; 71.543^{\circ} \mathrm{W}$ & $38.842^{\circ} \mathrm{N} ; 71.617^{\circ} \mathrm{W}$ & 4.49 & $\mathrm{DR}$ \\
\hline 114 & 424.3 & $38.877^{\circ} \mathrm{N} ; 71.563^{\circ} \mathrm{W}$ & $38.643^{\circ} \mathrm{N} ; 72.032^{\circ} \mathrm{W}$ & 26.00 & SR \\
\hline 115 & 425.3 & $38.863^{\circ} \mathrm{N} ; 71.625^{\circ} \mathrm{W}$ & $38.593^{\circ} \mathrm{N} ; 72.163^{\circ} \mathrm{W}$ & 29.95 & $\mathrm{DR}$ \\
\hline Day & Cast. & Est. Ring Center & Station Position & Dist. RC. & $(N M)$ \\
\hline 115 & 425.5 & $38.863^{\circ} \mathrm{N} ; 71.625^{\prime} \mathrm{W}$ & $38.442^{\circ} \mathrm{N} ; 72.510^{\circ} \mathrm{W}$ & 48.58 & SR \\
\hline 110 & 426.2 & $38.870^{\circ} \mathrm{N} ; 71.702^{\prime} \mathrm{W}$ & $38.538^{\circ} \mathrm{N} ; 72.335^{\circ} \mathrm{W}$ & 35.71 & $\mathrm{DR}$ \\
\hline 110 & $4<0.5$ & $38.870^{\circ} \mathrm{N} ; 71.702^{\prime} \mathrm{W}$ & $38.633^{\circ} \mathrm{N} ; 72.450^{\circ} \mathrm{W}$ & 37.79 & SS \\
\hline 117 & 427.2 & $38.877^{\circ} \mathrm{N} ; 71.777^{\circ} \mathrm{W}$ & $38.847^{\circ} \mathrm{N} ; 71.620^{\circ} \mathrm{W}$ & 7.54 & SR \\
\hline 118 & 428.2 & $38.883^{\circ} \mathrm{N} ; 71.853^{\circ} \mathrm{W}$ & $38.880^{\circ} \mathrm{N} ; 71.680^{\circ} \mathrm{W}$ & 8.16 & SR \\
\hline 119 & 429.1 & $38^{\circ} 890^{\circ} \mathrm{N} ; 71: 928^{\circ} \mathrm{W}$ & $39.347^{\circ} \mathrm{N} ; 71.883^{\circ} \mathrm{W}$ & 27.48 & $\mathrm{DR}$ \\
\hline 120 & 430.1 & $38.897^{\circ} \mathrm{N} ; 72.005^{\circ} \mathrm{W}$ & $39.340^{\circ} \mathrm{N} ; 71.513^{\circ} \mathrm{W}$ & 35.09 & SR \\
\hline 120 & 430.5 & $38.897^{\circ} \mathrm{N} ; 72.005^{\circ} \mathrm{W}$ & $39.833^{\circ} \mathrm{N} ; 71.568^{\circ} \mathrm{W}$ & 59.74 & SR \\
\hline $1\llcorner 1$ & 501.3 & $38.943^{\circ} \mathrm{N} ; 72.080^{\circ} \mathrm{W}$ & $38.920^{\circ} \mathrm{N} ; 71.957^{\circ} \mathrm{W}$ & 5.86 & SS \\
\hline 122 & 502.2 & $38.900^{\circ} \mathrm{N} ; 72.080^{\circ} \mathrm{W}$ & $39.510^{\circ} \mathrm{N} ; 70.930^{\circ} \mathrm{W}$ & 64.80 & SR \\
\hline $1<2$ & 502.3 & $38.900^{\circ} \mathrm{N} ; 72.080^{\circ} \mathrm{W}$ & $39.557^{\circ} \mathrm{N} ; 70.885^{\circ} \mathrm{W}$ & 68.10 & DR \\
\hline 122 & 502.5 & $39.900^{\circ} \mathrm{N} ; 72.080^{\circ} \mathrm{W}$ & $39.495^{\circ} \mathrm{N} ; 70.970^{\circ} \mathrm{W}$ & 62.76 & SR \\
\hline 122 & 502.7 & $38.900^{\circ} \mathrm{N} ; 72.080^{\circ} \mathrm{W}$ & $39.529^{\circ} \mathrm{N} ; 70.813^{\circ} \mathrm{W}$ & 69.95 & SS \\
\hline
\end{tabular}

\section{MIDWATER FISHES}

Principal Investigator: Richard Backus

Lruise Participants: James Craddock, Mary Ann Daher, John Pirie and Joseph Wroblewski

Woods Hole Uceanographic Institution and Dalhousie University

During UCEAivUS Cruise No. 118, the MOCNESS-20 trawl system was fished twelve times. Each lowering was to 1000 meters using 5 nets, the first net fisning $U-1000$ meters and each successive net fishing at 250-meter intervals on the way back to the surface. 
Four lowerings were made in Slope Water; four lowerings were made within $25 \mathrm{~km}$ of the center of warm core ring 82B; and four lowerings were made in the high velocity region of ring $82 \mathrm{~B}(50-75 \mathrm{~km}$ from its center).

Tnese collections are summarized in the accompanying Table 1.

Likewise, fifteen surface collections were made at night with a 1 meter neuston net at two Slope water stations and at two ring stations;

specifically, 6 tows along the track of MOC-1D-163 (2 hrs); 4 along the track of MUC-2U-13 ( 2 hrs); 2 along the track of MOC-1D-168 (1.45 hrs); and 3 along the track of MUC-20-18 (2.33 hrs).

\section{ZUUPLANKTON SPATIAL PATTERNS}

Principal Investigator: Peter $\mathrm{H}$. Wiebe

Cruise Participants: Steven Boyd, Alfred Morton, Valerie Barber and Peter $\mathrm{H}$. Wiebe

Woods Hole Oceanographic Institution

Our objectives in this first of four time-series cruises to Gulf Stream warm core rings were to sample the macro-zooplankton at station locations placed from the center of ring $82 \mathrm{~B}$ out into the adjacent Slope Water in order to provide a picture of the vertical structure in the upper 1000 meters across the ring for a variety of species, especially the euphausiids, and to characterize the diel vertical movements of these species as well as the total zooplankton biomass. In addition, we intended to collect individuals of a number of euphausiid species for biochemical analysis in order to study the effects of spatial and time-course changes in rings on their physiological and biochemical properties. During the joint ship experiments in the ring center and high velocity region, we intended to look at the coarse-scale spatial structure of the zooplankton in the upper 100 meters associated with intrusions at the ring boundary and with internal wave patterns in the ring core.

We used a double MUCNESS-1 to sample the zooplankton in the upper 1000 meters. Un each 3-hour haul, we generally obtained 8 samples integrating 100 meter intervals from 1000 to 200 meters and 8 samples integrating 25 meter intervals from 200 to 0 meters. A total of 15 hauls were made, of which 13 will probably be suitable for documenting ring zooplankton structure. A total of 231 samples were collected on these tows. We were unable to take the tows to study the coarse-scale patterns of zooplankton primarily because of high winds and waves during both of the days set aside for the joint experiments. Table 1 presents details of times, dates, positions, etc., of the double MUCiveSS tows; Table 2 gives the distance of each tow from the ring center using positions listed in narrative Table 1).

To help correlate our zooplankton data with the findings of phytoplankton investigators on ship 2 ( $R / V$ KNORR), we made a number of 5-7iter Niskin bottle casts in the upper 100 to 200 meters for analysis of chlorophyl1. This chlorophyll data will also be used in conjunction with pyranometer data 
Backus et al.

Table 1. Summary of MOCNESS-20 collections, Oceanus Cruise 118

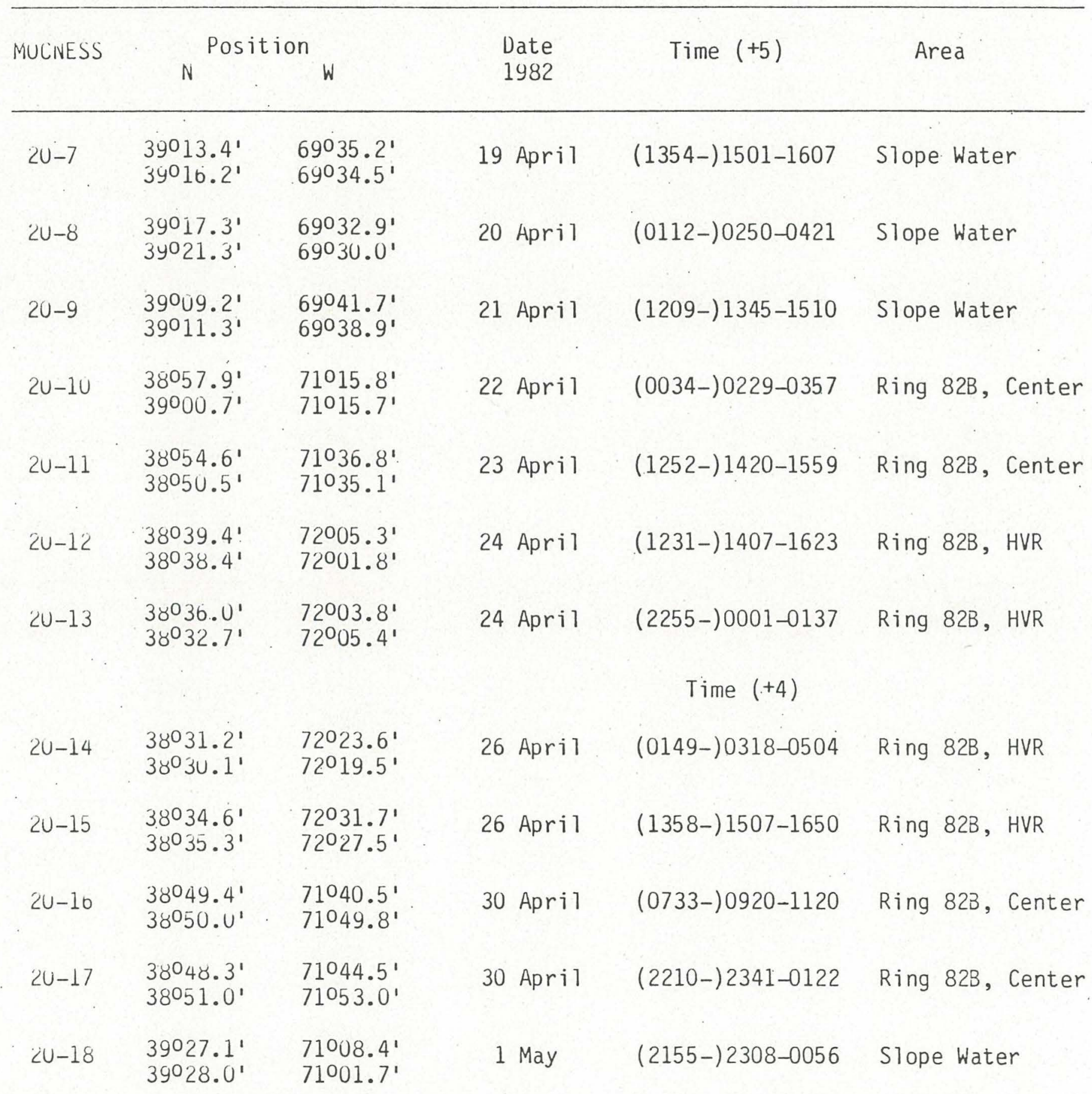


GULF STREAM RING TIME SERIES CRUISE

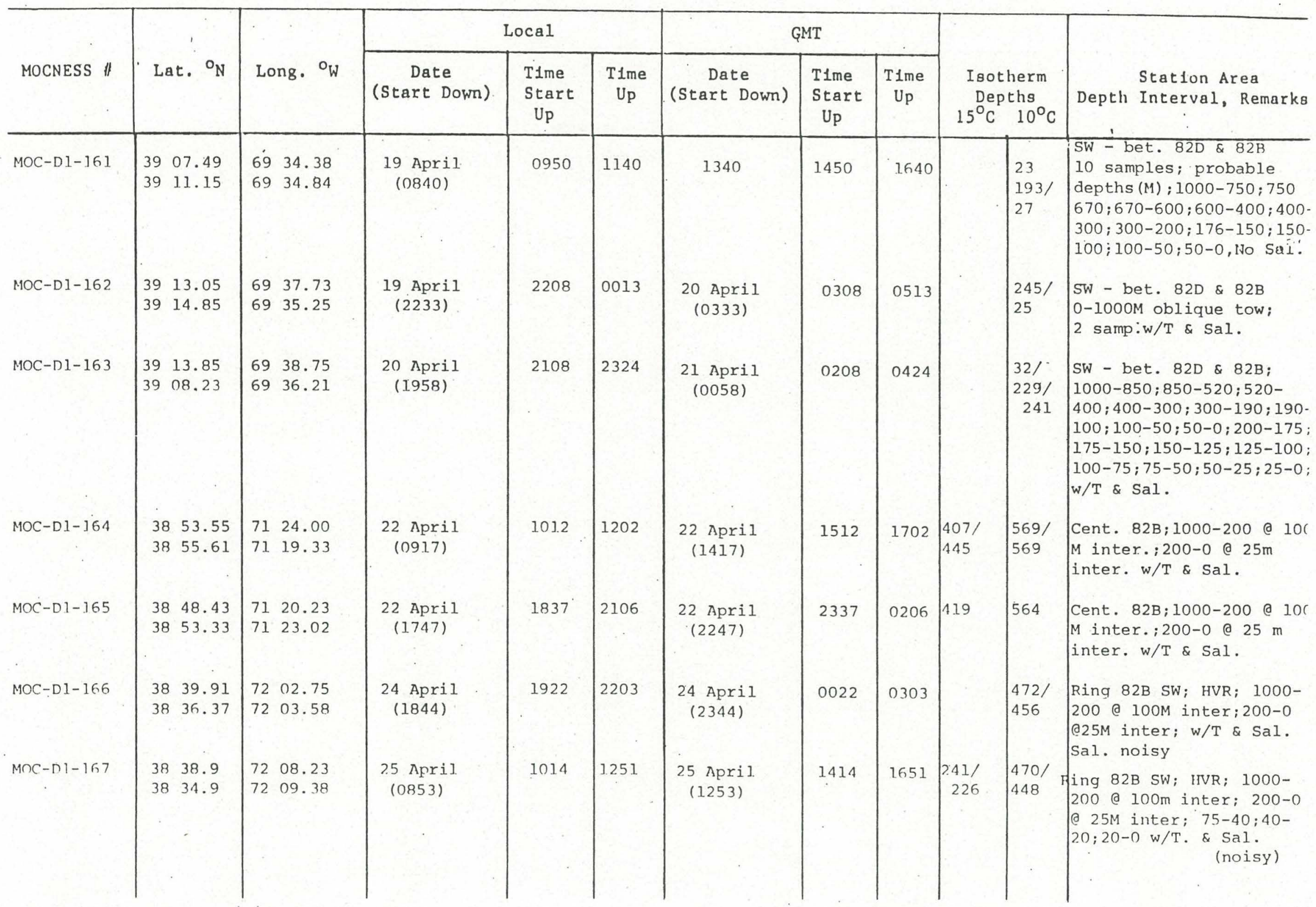


WARM CORE

GULF STREAM RING TIME SERIES CRUISE

\begin{tabular}{|c|c|c|c|c|c|c|c|c|c|c|c|}
\hline \multirow[b]{2}{*}{ MOCNESS } & \multirow{2}{*}{ Lat. ${ }^{\circ} \mathrm{N}$} & \multirow[b]{2}{*}{ Long. ${ }^{o} W$} & \multicolumn{3}{|c|}{ Local } & \multicolumn{3}{|c|}{ GMT } & \multirow{2}{*}{\multicolumn{2}{|c|}{$\begin{array}{l}\text { Isotherm } \\
\text { Depths } \\
15^{\circ} \mathrm{C \quad} 10^{\circ} \mathrm{C}\end{array}$}} & \multirow[b]{2}{*}{$\begin{array}{cc}\text { Station Area } \\
\text { Depth Interval, Remark } \\
\end{array}$} \\
\hline & & & $\begin{array}{l}\text { Date } \\
\text { (St rt Down) }\end{array}$ & $\begin{array}{l}\text { TIme } \\
\text { Start } \\
\text { Up }\end{array}$ & $\underset{\text { Up }}{\text { TIme }}$ & $\begin{array}{c}\text { Date } \\
\text { (Start Down) }\end{array}$ & $\begin{array}{l}\text { TIme } \\
\text { Start } \\
\text { Up }\end{array}$ & $\begin{array}{l}\text { TIme } \\
\text { Up }\end{array}$ & & & \\
\hline MOC-D1-168 & $\begin{array}{ll}38 & 26.78\end{array}$ & 7230.89 & $\begin{array}{l}26 \text { April } \\
(2145)\end{array}$ & 2247 & 0106 & $\begin{array}{l}27 \text { April } \\
(0145)\end{array}$ & 0247 & 0506 & 15 & $\begin{array}{l}287 \\
344\end{array}$ & $\begin{array}{l}\text { Ring 82B SW; HVR; } \\
\text { 1000-200 @ 100M inter; } \\
200-0 \text { \& 25M inter; ok } \\
\text { w/T. \& Sal. }\end{array}$ \\
\hline MOC-D1-169 & $\begin{array}{ll}38 & 33.07 \\
38 & 34.35\end{array}$ & $\begin{array}{ll}72 & 21.75 \\
72 & 32.08\end{array}$ & $\begin{array}{l}26 \text { April } \\
(1004)\end{array}$ & 1113 & 1314 & $\begin{array}{l}26 \text { April } \\
(1404)\end{array}$ & 1513 & 1714 & 107 & $\begin{array}{l}391 / \\
357\end{array}$ & $\begin{array}{l}\text { Ring 82B SW; HVR; 1000- } \\
200 \text { a } 100 \mathrm{M} \text { inter; 200- } \\
0 \text { @ 25M inter.; 50-0 } \\
\text { w/T. \& Sal. }\end{array}$ \\
\hline MOC-D $1-170$ & $\begin{array}{ll}39 & 22.6 \\
39 & 21.5\end{array}$ & $\begin{array}{l}71: 52.6 \\
7153.5\end{array}$ & $\begin{array}{l}29 \text { April } \\
(1057)\end{array}$ & 1157 & 1447 & $\begin{array}{l}29 \text { April } \\
\text { (1457) }\end{array}$ & 1557 & 1947 & & $\begin{array}{r}330 / \\
347\end{array}$ & $\begin{array}{l}\text { Ring 82B NW; HVR; Joint } \\
\text { Ship Exp.; 1000-200 } \\
\text { 100M inter; } 200-0 \text { @ } \\
25 \mathrm{M} \text { inter; ok w/T \& Sa: }\end{array}$ \\
\hline MOC-D 1-171 & $\begin{array}{ll}39 & 24.09 \\
39 & 20.41\end{array}$ & $\begin{array}{ll}71 & 27.68 \\
71 & 30.75\end{array}$ & $\begin{array}{l}29 \text { April } \\
(2256)\end{array}$ & 0017 & 0224 & $\begin{array}{l}30 \text { April } \\
\text { (0256) }\end{array}$ & 0417 & 0624 & & $\begin{array}{l}350 / \\
357\end{array}$ & $\begin{array}{l}\text { Ring 82B NW; HVR; Joint } \\
\text { Ship Exp.; 1000-200: @ } \\
100 \mathrm{~m} \text { inter; 200-0 @ } 25 \mathrm{~m} \\
\text { inter.; ok w/T. \& Sal. }\end{array}$ \\
\hline MOC-D1-172 & $\begin{array}{ll}38 & 55.7 \\
38 & 51.25\end{array}$ & $\begin{array}{ll}71 & 39.8 \\
71 & 31.48\end{array}$ & $\begin{array}{l}30 \text { April } \\
(1305)\end{array}$ & 1418 & 1622 & $\begin{array}{l}30 \text { April } \\
\text { (1705) }\end{array}$ & 1818 & 2022 & $\begin{array}{l}458 / \\
427\end{array}$ & $\begin{array}{l}579 / \\
556\end{array}$ & $\begin{array}{l}\text { Ring 82B Center; 1000- } \\
200 \text { a 100m inter; 200- } \\
\text { o a } 25 \mathrm{~m} \text { inter; ok w/ } \\
\text { T \& Sal. }\end{array}$ \\
\hline$M O C-D i-173$ & $\begin{array}{ll}38 & 19.1 \\
38 & 48.3\end{array}$ & $\begin{array}{ll}71 & 31.8 \\
71 & 11.5\end{array}$ & $\begin{array}{l}30 \text { nprji. } \\
(1830)\end{array}$ & 1928 & 2.133 & $\begin{array}{c}30 \text { nprit } \\
(2230)\end{array}$ & 2.328 & 0.1 .33 & $\begin{array}{l}367 / \\
386\end{array}$ & $\begin{array}{l}521 / \\
535\end{array}$ & $\begin{array}{l}\text { Ring } 82 \mathrm{~B} \text { Center; } 1000- \\
200 \text { a } 100 \mathrm{~m} \text { inter; } 200- \\
0 \text { at } 25 \mathrm{~m} \text { inter; } 100-0 \\
\text { No T. or Sal. }\end{array}$ \\
\hline
\end{tabular}


WARM CORE

GULF STREAM RING TIME SERIES CRUISE

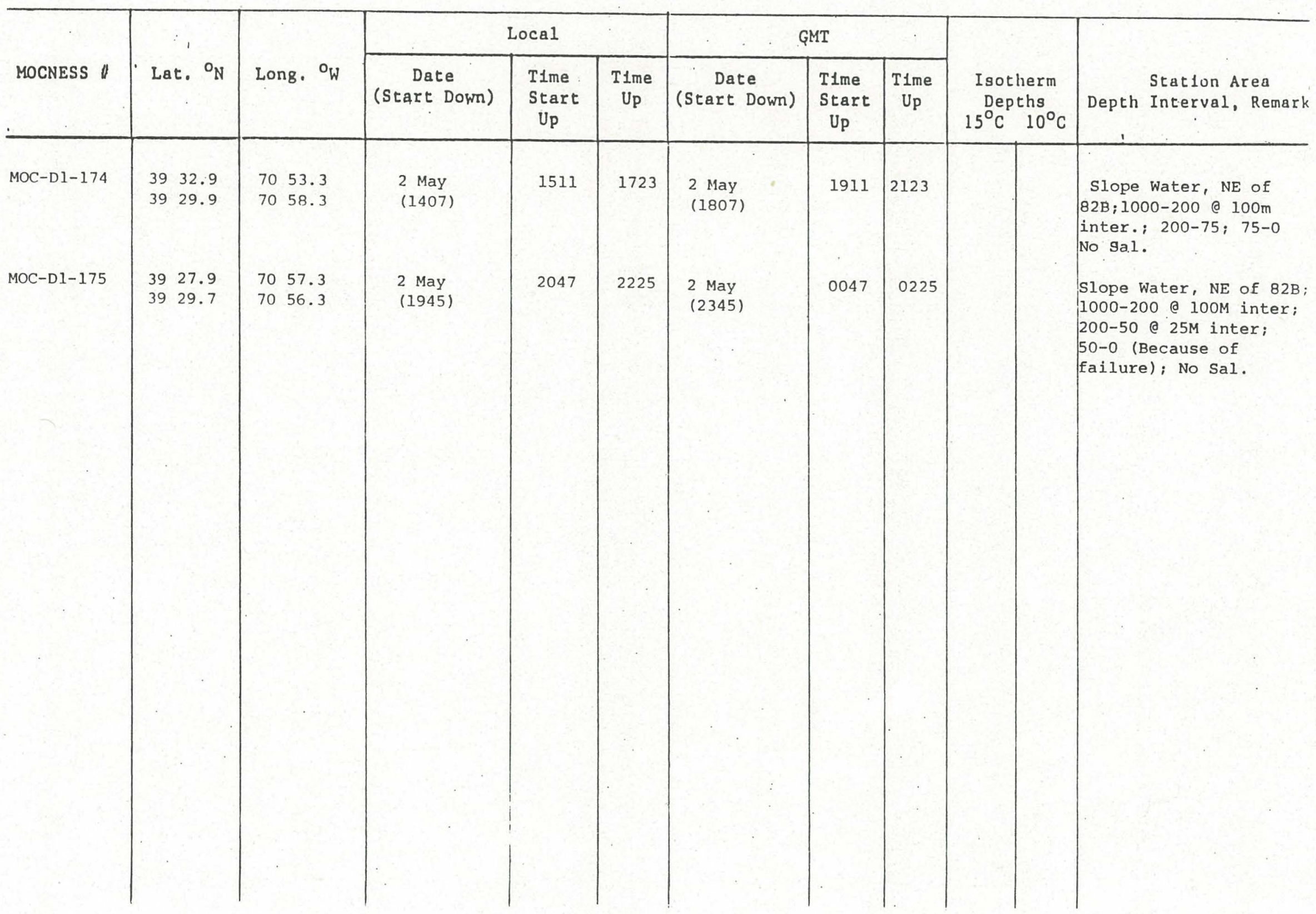


collected by us on the OCEANUS to calculate downwelling light levels in the upper 1000 meters. These light data were collected at 10 -minute intervals for the duration of the cruise with an MR-5 pyranometer (Hollis Observatory product). Uuring each station, a chlorocast was made in the euphotic zone to determine tne standing stocks of chlorophyll a and phaeophytin. Generally, samples were taken in ten-meter intervals from the surface to 100 meters. On four separate occasions, samples were taken to 200 meters to establish changes below 100 meters. In addition, samples taken by the "radon group" were analyzed for chlorophyll on 3 occasions (Table 3). In general, chlorocast positions bracketed double MOCNESS tows and offered reasonable estimations of chlorophyll pigment standing stocks (see Narrative, Figure 1)

Euphausiias from ten MOCNESS-20 trawls and three neuston tows were preserved for carbon, nitrogen and hydrogen analysis, lipid fractionation or chloropnyll pigment levels. A total of eighteen species were processed with emphasis being placed on particular indicator species; i.e., Euphausia krohnii, Nematoscelis megalops, and Euphausia brevis. (Table 4).

\section{Wiebe et al.}

Table 2.

\begin{tabular}{|c|c|c|c|}
\hline $\mathrm{MOC}$ & & $\begin{array}{l}\text { Dist. to } \\
\text { Ring Center } \\
\text { NM }\end{array}$ & $\begin{array}{l}\text { Dist. to } \\
\text { Ring Center } \\
\text { KM }\end{array}$ \\
\hline 161 & $\begin{array}{l}S \\
F\end{array}$ & $\begin{array}{l}80.4 \\
80.8\end{array}$ & $\begin{array}{l}149.0 \\
149.8\end{array}$ \\
\hline 162 & $\begin{array}{l}S \\
F\end{array}$ & $\begin{array}{l}79.1 \\
81.5\end{array}$ & $\begin{array}{l}146.6 \\
150.9\end{array}$ \\
\hline 163 & $\begin{array}{l}S \\
F\end{array}$ & $\begin{array}{l}78.4 \\
79.2\end{array}$ & $\begin{array}{l}145.2 \\
146.8\end{array}$ \\
\hline 164 & $\begin{array}{l}S \\
F\end{array}$ & $\begin{array}{l}1.6 \\
4.3\end{array}$ & $\begin{array}{l}3.0 \\
8.1\end{array}$ \\
\hline 165 & $\begin{array}{l}S \\
F\end{array}$ & $\begin{array}{l}4.3 \\
1.1\end{array}$ & $\begin{array}{l}7.9 \\
2.1\end{array}$ \\
\hline 166 & $\begin{array}{l}S \\
F\end{array}$ & $\begin{array}{l}25.9 \\
28.3\end{array}$ & $\begin{array}{l}48.0 \\
52.5\end{array}$ \\
\hline 167 & $\begin{array}{l}S \\
F\end{array}$ & $\begin{array}{l}27.2 \\
30.1\end{array}$ & $\begin{array}{l}50.4 \\
55.7\end{array}$ \\
\hline 168 & $\begin{array}{l}S \\
F\end{array}$ & $\begin{array}{l}45.8 \\
39.6\end{array}$ & $\begin{array}{l}84.9 \\
73.3\end{array}$ \\
\hline
\end{tabular}




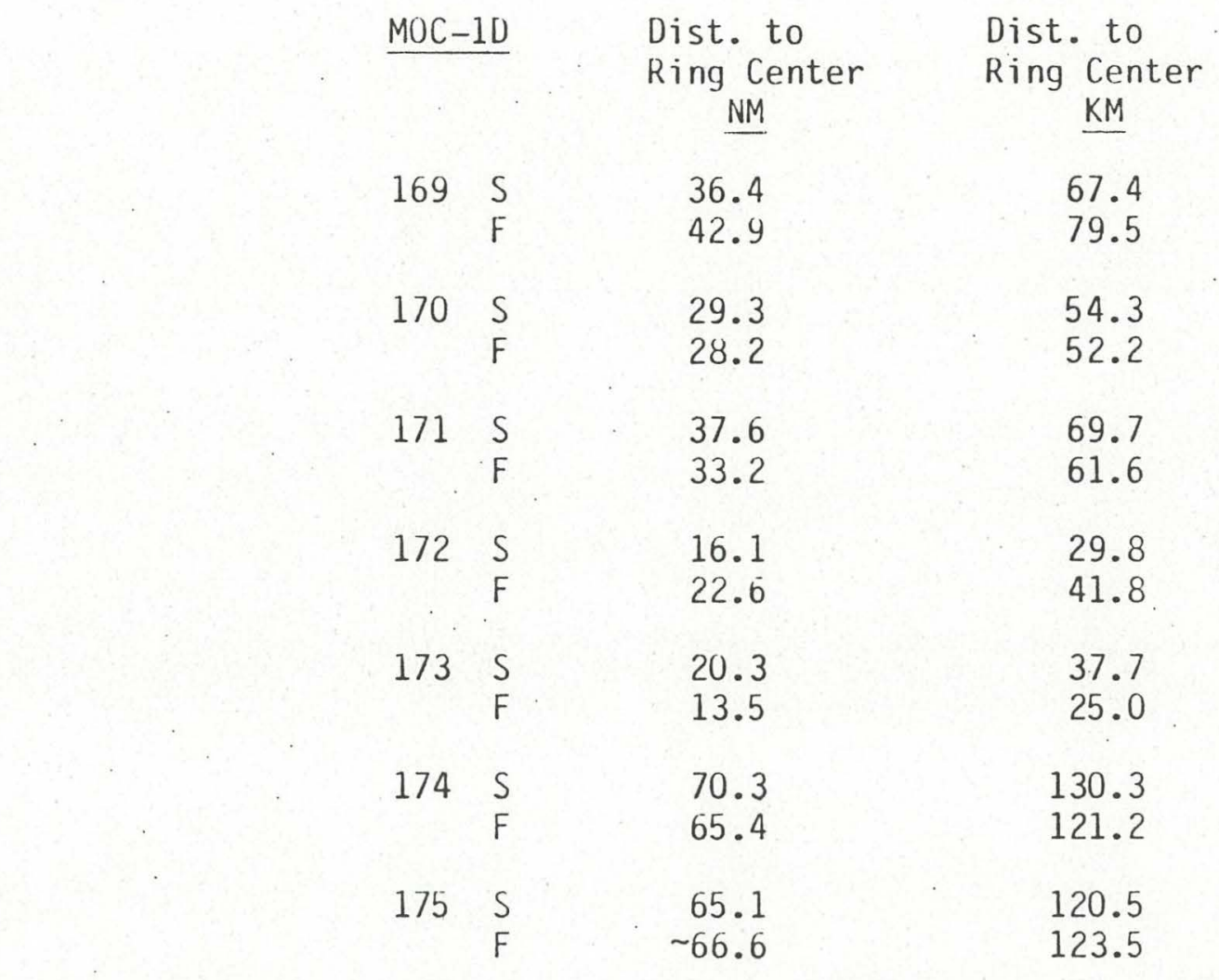

Wiebe, et al.

Table 3. Chlorophyll Cast Data

\begin{tabular}{|c|c|c|c|c|c|c|c|c|c|}
\hline $\begin{array}{l}\text { Cast } \\
\text { No. }\end{array}$ & Date & Time & Region & Lat. & Long. & \multicolumn{2}{|c|}{$\begin{array}{l}\text { Dist. } \\
\text { from R.C. } \\
\text { Km./Kt. }\end{array}$} & Depth & Int. \\
\hline 1 & $4 / 19 / 82$ & 0530 & SW & $39.07 .5^{\circ} \mathrm{N}$ & $69.33 .3^{\circ} \mathrm{W}$ & 150.5 & 81.3 & $140 \mathrm{~m}$ & $10 \mathrm{~m}$ \\
\hline 2 & $4 / 20 / 82$ & 1400 & SW & $39.16 .1^{\circ} \mathrm{N}$ & $69.34 .2^{\circ} \mathrm{W}$ & 152.5 & 82.3 & $100 \mathrm{~m}$ & $10 \mathrm{~m}$ \\
\hline 3 & $4 / 21 / 82$ & 1100 & SW & $39.09 .0^{\circ} \mathrm{N}$ & $69.42 .0^{\circ} \mathrm{W}$ & 143.7 & 77.5 & $200 \mathrm{~m}$ & $10 \mathrm{~m}$. \\
\hline 4 & $4 / 22 / 82$ & 0730 & $\mathrm{RC}$ & $38.51 .5^{\circ} \mathrm{N}$ & $71.22 .9^{\circ} \mathrm{W}$ & 1.3 & 0.7 & $200 m$ & $10 \mathrm{~m}$ \\
\hline 5 & $4 / 23 / 82$ & 1200 & $\mathrm{RC}$ & $38.53 .4^{\circ} \mathrm{N}$ & $71.36 .5^{\circ} \mathrm{W}$ & 5.6 & 3.0 & $200 m$ & $20 \mathrm{~m}$ \\
\hline 0 & $4 / 24 / 82$ & 1200 & HVR & $38.39 .0^{\circ} \mathrm{N}$ & $72.05 .5^{\circ} \mathrm{W}$ & 51.6 & 27.8 & $100 \mathrm{~m}$ & $10 \mathrm{~m}$ \\
\hline 7 & $4 / 25 / 82$ & 1721 & HVK & $38.26 .0^{\circ} \mathrm{N}$ & $72.30 .3^{\circ} \mathrm{W}$ & 91.1 & 48.6 & $100 \mathrm{~m}$ & $10 \mathrm{~m}$ \\
\hline 8 & $4 / 20 / 82$ & 0900 & RE & $38.33 .0^{\circ} \mathrm{N}$ & $72.21 .0^{\circ} \mathrm{W}$ & 66.6 & 35.9 & $100 \mathrm{~m}$ & $10 \mathrm{~m}$ \\
\hline y & $4 / 27 / 82$ & 1540 & $\mathrm{RC}$ & $38.51 .0^{\circ} \mathrm{N}$ & $71.37 .0^{\circ} \mathrm{W}$ & 14.2 & 7.6 & $100 \mathrm{~m}$ & $10 \mathrm{~m}$ \\
\hline 10 & $4 / 28 / 82$ & 1400 & $\mathrm{RC}$ & $38.53 .0^{\circ} \mathrm{N}$ & $71.41 .0^{\circ} \mathrm{W}$ & 14.7 & 7.9 & $200 \mathrm{~m}$ & $20 \mathrm{~m}$ \\
\hline 11 & $4 / 29 / 82$ & 0900 & HVK & $39.22 .0^{\circ} \mathrm{N}$ & $71.51 .0^{\circ} \mathrm{W}$ & 53.4 & 28.8 & $100 \mathrm{~m}$ & $10 \mathrm{~m}$ \\
\hline $1<$ & $4 / 30 / 82$ & 1630 & $\mathrm{RC}$ & $38.50 .0^{\circ} \mathrm{N}$ & $71.34 .0^{\circ} \mathrm{W}$ & 38.6 & 20.8 & $220 m$ & $10 \mathrm{~m}$ * \\
\hline 13 & $5 / 1 / 82$ & 2145 & SW & $39.27 .0^{\circ} \mathrm{N}$ & $71.08 .4^{\circ} \mathrm{K}$ & 101.3 & 54.7 & $100 \mathrm{~m}$ & $10 \mathrm{~m}$ \\
\hline
\end{tabular}

*Additional radon samples

$\mathrm{SW}=$ Slope Water; $\mathrm{RC}=$ Ring Center; HVR = High Velocity Region; RE = Ring Edge 


\section{Wiebe et al.}

Table 4. Number of Individuals Collected. For Biochemical Analysis According to Intended Treatment

\begin{tabular}{|c|c|c|c|}
\hline Species & Acetone & $\mathrm{CHN}$ & Chlor-Meth \\
\hline E. krohnii & 19 & 72 & 44 \\
\hline E. mutica & 1 & & \\
\hline E. brevis & 1 & 6 & 10 \\
\hline E. hemigibba & 8 & 6 & \\
\hline $\bar{E}$. gibboides & 1 & & 1 \\
\hline E. americana & & 2 & \\
\hline N. megalops & 10 & 6 & 13 \\
\hline N. microps & 2 & 4 & 2 \\
\hline $\bar{N}$ attantica & 2 & & 1 \\
\hline N. tenella & 2 & 5 & 1 \\
\hline T. monocantha & 1 & & \\
\hline T. aequalis & 4 & 10 & 3 \\
\hline T. acutifrons & 1 & & \\
\hline$\overline{T_{0}}$ obtusifrons & 1 & & \\
\hline T. gregaria & & 2 & \\
\hline N. flexipes & 4 & 6 & 3 \\
\hline N. sexspinosus & & & 1 \\
\hline S. elongatum & 1 & 5 & 2 \\
\hline
\end{tabular}


III.SCIENTIF IC OBSERVATION LOG

Sta. Up. No. Start Latitude Longitude Descriptor

Invest.

$10419.10400 \quad 39.08 .41^{\circ} \mathrm{N} 69.31 .28^{\circ} \mathrm{W}$ Shallow Radon Cast

Orr;

Setser

$1 \quad$ U419.2 $0515 \quad 39.07 .72^{\circ} \mathrm{N} \quad 69.32 .00^{\circ} \mathrm{W}$ 5-L Chlorophyll Cast No. 1

Boyd

I $\quad 0419.30840 \quad 39.07 .49^{\circ} \mathrm{N} \quad 69.34 .38^{\circ} \mathrm{W} \quad$ MOC-1D-161

Wiebe

$1 \quad 0419.4 \quad 1500 \quad 39.14 .78^{\circ} \mathrm{N} \quad 69.34 .94^{\circ} \mathrm{W} \quad$ MOC-20-7

Craddock

$1 \quad 0419.5 \quad 1714 \quad 39.15 .56^{\circ} \mathrm{N} \quad 69.34 .76^{\circ} \mathrm{W}$ LVFS No. 1

Bishop

I $\quad 0419.6 \quad 2210 \quad 39.13 .05^{\circ} \mathrm{N} \quad 69.37 .73^{\circ} \mathrm{W} \quad$ MOC-1D-162

Wiebe

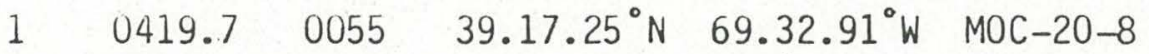

Craddock

$1 \quad 0420.1$ uguo $39.16 .6^{\circ} \mathrm{N} \quad 69.33 .5^{\circ} \mathrm{W}$ Six Shooter to $1000 \mathrm{M}$

Orr

1. $\quad 0420.2 \quad 1346 \quad 39.16 .6^{\circ} \mathrm{N} \quad 69.33 .5^{\circ} \mathrm{W}$ 5-L Chlorophyll Cast No. 2

Boyd

$1 \quad 0420.3 \quad 1420$

$39.15 .77^{\circ} \mathrm{N}$

$69.34 .66^{\circ} \mathrm{W}$ Net Tow

Boyd

1. $\quad 0420.4 \quad 1520$

$39.15 .2^{\circ} \mathrm{N}$

69.36. $1^{\circ} \mathrm{W}$ STD/Rosette, 30L

Orr

$\begin{array}{lll}1 & 0420.5 & 1945\end{array}$

$39.13 .85^{\circ} \mathrm{N}$

$69.38 .75^{\circ} \mathrm{W}$ MOC-1D-163

Wiebe

I $0420.6 \quad 2340$

$39.08 .07^{\circ} \mathrm{N} \quad 69.36 .17^{\circ} \mathrm{W}$ LVFS No. 2

Bishop

$10421.1 \quad 1115$

$39.09 .37^{\circ} \mathrm{N}$

69.41.59 $\mathrm{W}$ 5-L. Chlorophyl1 Cast No. 3

Boyd

$10421.2 \quad 1200$

$39.09 .13^{\circ} \mathrm{N} \quad 69.41 .62^{\circ} \mathrm{W} \quad$ MOC-20-9

Craddock

$1 \quad 0.421 .3 \quad 1530$

$39.10 .9^{\circ} \mathrm{N}$

69.38. $7^{\circ} \mathrm{W}$ Radium, Radon Cast to $1000 \mathrm{M}$

Orr

$2 \quad 0422.1 \quad 0020 \quad 38.57 .62^{\circ} \mathrm{N} \quad 71.15 .50^{\circ} \mathrm{W} \quad$ MOC-20-10

Craddock

$2 \quad 0422.2 \quad 0600 \quad 38.50 .4^{\circ} \mathrm{N} \quad 71.23 .1^{\circ} \mathrm{W}$ 500M Radon Hydrowire

Orr;
Setser

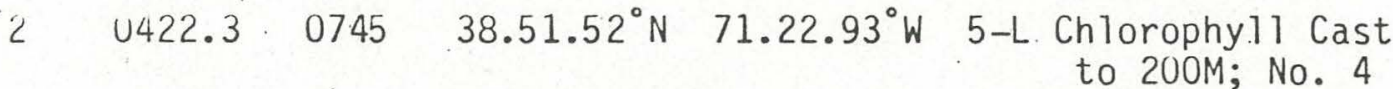

Boyd

¿ $\quad 0422.4 \quad 0915 \quad 38.53 .55^{\circ} \mathrm{N} \quad 71.24 .0^{\circ} \mathrm{W} \quad$ MOC-1D-164

Wiebe 
$\begin{array}{llllllll}\text { Sta. Op. No. } & \begin{array}{l}\text { Start } \\ \text { Time }\end{array} & \text { Latitude } & \text { Longitude } & \text { Descriptor } & \text { Invest. } \\ 2 & 0422.5 & 1210 & 38.55 .61^{\circ} \mathrm{N} & 71.19 .33^{\circ} \mathrm{W} & \text { LVFS No. 3 } & \text { Bishop } \\ 2 & 0422.6 & 1943 & 38.48 .43^{\circ} \mathrm{N} & 71.20 .23^{\circ} \mathrm{W} & \text { MOC-1D-165 } & \text { Wiebe } \\ 2 & 0422.7 & 2223 & 38.54 .39^{\circ} \mathrm{N} & 71.23 .26^{\circ} \mathrm{W} & \text { Six Shooter/STD } & \text { Orr } \\ 2 & 0423.1 & 0300 & 38.50 .37^{\circ} \mathrm{N} & 71.26 .24^{\circ} \mathrm{W} & \text { STD Rosette } & \text { Orr } \\ 2 & 0423.2 & 0645 & 38.48 .3^{\circ} \mathrm{N} & 71.31 .1^{\circ} \mathrm{W} & \text { LVFS No. 4 } & \text { Bishop } \\ 2 & 0423.3 & 1211 & 38.53 .4^{\circ} \mathrm{N} & 71.36 .5^{\circ} \mathrm{W} & \text { 5-L Chlorophyll Cast } & \text { Boyd }\end{array}$

$2 \quad 0423.4 \quad 1250 \quad 38.54 .22^{\circ} \mathrm{N} \quad 71.36 .63^{\circ} \mathrm{W}$ MOC-20-11 Craddock

2. $\quad 0423.5 \quad 1630 \quad 38.50 .5^{\circ} \mathrm{N} \quad 71.37 .0^{\circ} \mathrm{W}$ Deep Radon Cast to $2600 \mathrm{M}$ Orr

$\angle \quad 0423.62100 \quad 38.42 .57^{\circ} \mathrm{N} 71.42 .7^{\circ} \mathrm{W}$ Deep LVFS No. 5 Bishop

$3 \quad 0424.1 \quad 1200 \quad 38.39 .4^{\circ} \mathrm{N} \quad 72.05 .0^{\circ} \mathrm{W} 5-\mathrm{L}$ Chlorophyll Cast Boyd

3. $0424.21230 \quad 38.38 .4^{\circ} \mathrm{N} \quad 72.05 .0^{\circ} \mathrm{W}$ MOC-20-12 Craddock

$3 \quad 0424.3 \quad 1640 \quad 38.38 .61^{\circ} \mathrm{N} \quad 72.01 .91^{\circ} \mathrm{W}$ Shallow Radon Cast Orr

3. $\quad 424.4 \quad 1820 \quad 38.39 .91^{\circ} \mathrm{N} 72.02 .75^{\circ} \mathrm{W}$ MOC-1D-166 Wiebe

s $0424.52250 \quad 38.35 .98^{\circ} \mathrm{N} 72.03 .8^{\circ} \mathrm{W}$ MOC-20-13 Craddock

$3 \quad \quad 425.1 \quad U 324 \quad 38.33 .16^{\circ} \mathrm{N} 72.06 .71^{\circ} \mathrm{W}$ Shallow LVFS No.6. Bishop

$3 \quad 0425.20853 \quad 38.38 .8^{\circ} \mathrm{N} 72.08 .3^{\circ} \mathrm{W}$ MOC-1D-167 Wiebe

$3 \quad 0425.3 \quad 1300 \quad 38.35 .5^{\circ} \mathrm{N} \quad 72.09 .5^{\circ} \mathrm{W}$ Deep Radon Cast Orr

s $0425.41900 \quad 38.26 .04^{\circ} \mathrm{N} 72.30 .3^{\circ} \mathrm{W}$ 5-L Chlorophyll Cast Boyd

$3 \quad 042 b .5 \quad 1940 \quad 40.26 .04^{\circ} \mathrm{N} \quad 72.30 .3^{\circ} \mathrm{W}$ Shallow Radon Cast orr

$4 \quad 0425.0 \quad 2249 \quad 38.26 .6^{\circ} \mathrm{N} \quad 72.31 .1^{\circ} \mathrm{W}$ MOC-1D-168 Wiebe

$4 \quad 0426.1014938 .31 .2^{\circ} \mathrm{N} 72.23 .6^{\circ} \mathrm{W}$ MOC-20-14, Craddock

$4 \quad 0426.20520 \quad 38.30 .9^{\circ} \mathrm{N} 72.19 .4^{\circ} \mathrm{W}$ Deep Radon Cast Orr

4. U426.3 $0910 \quad 38.32 .9^{\circ} \mathrm{N} 72.20 .8^{\circ} \mathrm{W} 5$ - L Chlorophyll Cast Boyd 


\begin{tabular}{|c|c|c|c|c|c|c|}
\hline a. & Up. No. & $\frac{\text { Start }}{\text { Time }}$ & Latitude & Longitude & Descriptor & Invest. \\
\hline 4 & 0426.4 & 1005 & $38.31 .3^{\circ} \mathrm{N}$ & $72.21 .5 \mathrm{~W}$ & MOC-1D-169 & Wiebe \\
\hline 4 & 0426.5 & 1358 & $38.34 .5^{\circ} \mathrm{N}$ & $72.31 .7 ' \mathrm{~W}$ & MOC $-20-15$ & Craddock \\
\hline 4 & 0426.6 & 1800 & $38.38 .0^{\circ} \mathrm{N}$ & $72.27 .01 \mathrm{w}$ & Six-Shooter STD & Orr \\
\hline 5 & 0427.1 & 1521 & $38.51 .6^{\circ} \mathrm{N}$ & $71.37 .1 \mathrm{~W}$ & $\begin{array}{l}\text { 5-L Chlorophyll Cast } \\
\text { No. } 9\end{array}$ & Boyd \\
\hline 5 & 0427.2 & 1545 & $38.50 .8^{\circ} \mathrm{N}$ & $71.32 .2^{\prime} \mathrm{W}$ & Shallow Radon Cast & Orr \\
\hline 5 & 0428.1 & 0317 & $38.50 .5^{\circ} \mathrm{N}$ & $71.40 .9^{\prime} \mathrm{W}$ & LVFS No. 7 & Bishop \\
\hline 5 & 0428.2 & 1200 & $38.52 .8^{\circ} \mathrm{N}$ & $71.40 .8^{\prime} \mathrm{W}$ & Shallow Radon Cast & Orr \\
\hline 5 & 0428.3 & 1357 & $38.52 .8^{\circ} \mathrm{N}$ & $71.40 .8^{\prime} \mathrm{W}$ & $\begin{array}{c}\text { 5-L Chlorophy } 11 \text { Cast } \\
\text { No. } 10\end{array}$ & Boyd \\
\hline 6 & $042 y .1$ & 0535 & $38.52 .65^{\circ} \mathrm{N}$ & $71^{\circ} 39.15^{\prime} \mathrm{W}$ & Deep Radon Cast & Orr \\
\hline b & $042 y .2$ & 0838 & $38.52 .8^{\circ} \mathrm{N}$ & $71^{\circ} 40.8^{\prime} \mathrm{W}$ & $\begin{array}{l}\text { 5-L Chlorophyl1 Cast } \\
\text { No. } 11\end{array}$ & Boyd \\
\hline 6 & 0429.3 & 1054 & $39.20 .93^{\circ} \mathrm{N}$ & $71^{\circ} 53.9^{\prime} \mathrm{W}$ & MOC-1D-170 & Wiebe \\
\hline o & 0429.4 & 1704 & $39.22 .3^{\circ} \mathrm{N}$ & $71^{\circ} 39.7{ }^{\prime} \mathrm{W}$ & LVFS No. 8 & Bishop \\
\hline b & $042 y .5$ & 2254 & $39.24 .1^{\circ} \mathrm{N}$ & $71^{\circ} 27.7^{\prime} \mathrm{W}$ & MOC-1D-171 & Wiebe \\
\hline b & 0430.1 & 0235 & $39.20 .4^{\circ} \mathrm{N}$ & $71^{\circ} 30.8^{\prime} \mathrm{W}$ & Shallow Radon Cast & Orr \\
\hline 7 & 0430.2 & 0730 & $38.49 .4^{\circ} \mathrm{N}$ & $71^{\circ} 40.5^{\prime} \mathrm{W}$ & MOC-20-16 & Craddock \\
\hline 7 & 0430.3 & 1304 & $38.55 .4^{\circ} \mathrm{N}$ & $71^{\circ} 39.4^{\prime} \mathrm{W}$ & MOC-1D-172 & Wiebe \\
\hline 7 & 0430.4 & 1645 & $38.50 .7^{\circ} \mathrm{N}$ & $.71 .33 .6^{\circ} \mathrm{W}$ & $\begin{array}{c}\text { 5-L Chlorophyll Cast } \\
\text { No. } 12\end{array}$ & Boyc \\
\hline 7 & 0430.5 & 1700 & $38.50 .7^{\circ} \mathrm{N}$ & $71.33 .6^{\circ} \mathrm{W}$ & Shallow Radon Cast & Or \\
\hline 7 & 0501.1 & 0200 & $38.51 .1^{\circ} \mathrm{N}$ & $71.53 .4^{\circ} \mathrm{W}$ & LVFS No. 9 & Bishor \\
\hline 7 & 0501.2 & 0915 & $38.53 .5^{\circ} \mathrm{N}$ & $71.55 .8^{\circ} \mathrm{W}$ & MULVFS -1 & Bishor \\
\hline 7. & 0501.3 & 1230 & $38.55 .2^{\circ} \mathrm{N}$ & $71.57 .4^{\circ} \mathrm{W}$ & Six Shooter STD & Or \\
\hline 8 & U5U1.4 & 2128 & $39.27 .0^{\circ} \mathrm{N}$ & $71.09 .2^{\circ} \mathrm{W}$ & $\begin{array}{c}\text { 5-L Chlorophyll Cast } \\
\text { No. } 13\end{array}$ & Boyd \\
\hline
\end{tabular}




\begin{tabular}{|c|c|c|c|c|c|c|}
\hline Sta. & Up. No. & $\begin{array}{l}\text { Start } \\
\text { Time }\end{array}$ & Latitude & Longitude & Descriptor & Invest. \\
\hline 8 & 0501.5 & 2153 & $39.27 .1^{\circ} \mathrm{N}$ & $71.08 .5^{\circ} \mathrm{W}$ & $M O C-20-18$ & Craddock \\
\hline 8 & 0502.1 & 0143 & $39.28 .6^{\circ} \mathrm{N}$ & $71.00 .7^{\circ} \mathrm{W}$ & LFVS-10 & Bishop \\
\hline 8 & 0502.2 & U6U0 & $39.30 .6^{\circ} \mathrm{N}$ & $70.55 \therefore$ & Shallow Radon Cast & Orr \\
\hline 8 & U502.3. & 1030 & $39.33 .4^{\circ} \mathrm{N}$ & $70.53 .1 " w$ & Deep Radon Cast & Orr \\
\hline 8 & 0502.4 & 1413 & $39.32 .9^{\circ} \mathrm{N}$ & $70.53 .2^{\circ} \mathrm{W}$ & $M O C-1 D-174$ & Wiobe \\
\hline 8 & $05 \cup 2.5$ & 1730 & $39.29 .7^{\circ} \mathrm{N}$ & $70.58 .19^{\circ} \mathrm{W}$ & Shallow Radon Cast & Orr \\
\hline 8 & 0502.6 & 1932 & $39.29 .6^{\circ} \mathrm{N}$ & $70.57 .1^{\circ} \mathrm{W}$ & $M O C-1 D-175$ & Wiebe \\
\hline 8 & 0502.7 & 2331 & $39.31 .7^{\circ} \mathrm{N}$ & $70.48 .8^{\circ} \mathrm{W}$ & Six Shooter STD & Orr \\
\hline
\end{tabular}


IV. XBT DIGITIZING LOG

\begin{tabular}{|c|c|c|c|c|c|c|c|}
\hline XBT & Date & $\begin{array}{c}\text { Time } \\
\text { (Local) } \\
\end{array}$ & Latitude & Longitude & $\begin{array}{l}\text { XBT } \\
\text { Surface } \\
\text { Temp }\left({ }^{\circ} \mathrm{C}\right) \\
\end{array}$ & $\begin{array}{l}\text { Depth } \\
15\end{array}$ & M) 10 \\
\hline 13 & $4 / 19 / 82$ & 0019 & $39.39 .7^{\circ} \mathrm{N}$ & $6.9 .1^{\circ} \mathrm{W}$ & 7.7 & $N P$ & $65: 222$ \\
\hline 14 & $4 / 19 / 82$ & 0115 & $39.30 .0^{\circ} \mathrm{N}$ & $t: .30 .5^{\circ} \mathrm{W}$ & 6.8 & $\mathrm{NP}$ & $58 ; 225$ \\
\hline 15 & $4 / 19 / 82$ & 0207 & $39.19 .8^{\circ} \mathrm{N}$ & $6 y^{\circ} 30.8^{\circ} \mathrm{W}$ & 7.5 & NP & $32 ; 215$ \\
\hline 10 & $4 / 19 / 82$ & 0300 & $39.09 .0^{\circ} \mathrm{N}$ & $69.31 .2^{\circ} \mathrm{W}$ & 8.6 & $\mathrm{NP}$ & $7 ; 210$ \\
\hline 17 & $4 / 19 / 82$ & 1717 & $39.15 .5^{\circ} \mathrm{N}$ & $69.34 .8^{\circ} \mathrm{W}$ & 8.0 & NP & $32 ; 235$ \\
\hline 18 & $4 / 20 / 82$ & 0840 & $39.20 .0^{\circ} \mathrm{N}$ & $69.30 .5^{\circ} \mathrm{W}$ & 10.1 & NP & $2 ; 222$ \\
\hline 19 & $4 / 21 / 82$ & U303 & $39.08 .4^{\circ} \mathrm{N}$ & $69.38 .8^{\circ} \mathrm{W}$ & 10.1 & NP & $2 ; 220$ \\
\hline 20 & $4 / 21 / 82$ & 1802 & $39.10 .5^{\circ} \mathrm{N}$ & $69.43 .1^{\circ} \mathrm{W}$ & 10.7 & NP & 241 \\
\hline 21 & $4 / 21 / 82$ & 1903 & $39.08 .7^{\circ} \mathrm{N}$ & $69.58 .6^{\circ} \mathrm{W}$ & 9.5 & NP & $66 ; 226$ \\
\hline 22 & $4 / 21 / 82$ & 2000 & $39.06 .7^{\circ} \mathrm{N}$ & $70.12 .8^{\circ} \mathrm{W}$ & 9.4 & NP & $80 ; 240$ \\
\hline 23 & $4 / 21 / 82$ & 2100 & $39.04 .4^{\circ} \mathrm{N}$ & $70.28 .8^{\circ} \mathrm{W}$ & 9.2 & NP & $80 ; 253$ \\
\hline 24 & $4 / 21 / 82$ & 2200 & $39.01 .8^{\circ} \mathrm{N}$ & $70.44 .5^{\circ} \mathrm{W}$ & 12.9 & NP & 364 \\
\hline 25 & $4 / 21 / 82$ & 2300 & $38.59 .0^{\circ} \mathrm{N}$ & $71.00 .5^{\circ} \mathrm{W}$ & 15.7 & 272 & 470 \\
\hline $\begin{array}{l}26 \\
27\end{array}$ & $\begin{array}{c}4 / 22 / 82 \\
N G\end{array}$ & UUUU & $38.57 .8^{\circ} \mathrm{N}$ & 71.15 . & 15.9 & 421 & 566 \\
\hline 28 & $4 / 22 / 82$ & 1539 & $38.49 .8^{\circ} \mathrm{N}$ & 71.20 .5 & 15.8 & 427 & 565 \\
\hline 29 & $4 / 23 / 82$ & 0549 & 38. & 71.29 & 15.8 & 425 & 560 \\
\hline 30 & $4 / 23 / 82$ & 1230 & 38.54 & 71.36 & 15.7 & 420 & 552 \\
\hline $3 i$ & $4 / 23 / 82$ & $1950^{\circ}$ & $38.50 .7^{\circ} \mathrm{N}$ & 71.38 . & 15.9 & 410 & 533 \\
\hline 32 & $4 / 23 / 82$ & 2109 & $38.42 .6^{\circ} \mathrm{N}$ & $71.42 .2^{\circ} \mathrm{W}$ & 15.7 & 363 & 529 \\
\hline $\begin{array}{l}33 \\
34\end{array}$ & $4 / 24 / 82$ & 0417 & $38.44 .0^{\circ} \mathrm{N}$ & $71.46 .7^{\circ} \mathrm{W}$ & 15.6 & 386 & 510 \\
\hline 35 & $4 / 24 / 82$ & 0755 & $38.46 .0^{\circ} \mathrm{N}$ & $71.50 .5^{\circ} \mathrm{W}$ & 15.8 & 375 & 502 \\
\hline 36 & $4 / 24 / 82$ & 1015 & $38.46 .9^{\circ} \mathrm{N}$ & $71.52 .4^{\circ} \mathrm{W}$ & 15.8 & 340 & 489 \\
\hline 37 & $4 / 24 / 82$ & 1115 & $38.41 .7^{\circ} \mathrm{N}$ & $72.00 .6^{\circ} \mathrm{W}$ & 15.0 & $0 ; 242$ & 450 \\
\hline 38 & $4 / 24 / 82$ & 1138 & $38.39 .0^{\circ} \mathrm{N}$ & $72.04 .9^{\circ} \mathrm{W}$ & 14.4 & $97 ; 238$ & 431 \\
\hline 39 & $4 / 24 / 82$ & $163 b$ & $38.34 .5^{\circ} \mathrm{N}$ & $72.01 .9^{\circ} \mathrm{W}$ & 15.7 & $243^{200}$ & 452 \\
\hline $4 U$ & $4 / 25 / 82$ & U233 & $38.33 .6^{\circ} \mathrm{N}$ & $72.06 .8^{\circ} \mathrm{W}$ & 14.9 & $60 ; 80 ; 112$ & 430 \\
\hline 41 & $4 / 25 / 82$ & 0043 & $38.36 .4^{\circ} \mathrm{N}$ & 72.07 & 15.5 & 218 & 448 \\
\hline 42 & $4 / 25 / 82$ & 1535 & $38.36 .1^{\circ} \mathrm{N}$ & 72.10 & 15.0 & 230 & 426 \\
\hline 43 & $4 / 25 / 82$ & 1721 & $38.30 .4^{\circ} \mathrm{N}$ & 72.2 & 13.8 & NP & 355 \\
\hline 44 & $4 / 25 / 82$ & 1758 & $38.25 .8^{\circ} \mathrm{N}$ & $72.27 .6^{\circ} \mathrm{W}$ & 16.0 & 12 & 282 \\
\hline 45 & $4 / 20 / 82$ & 0938 & $38.33 .1^{\circ} \mathrm{N}$ & $72.21 .1^{\circ} \mathrm{W}$ & 13.6 & $45 ; 189$ & 385 \\
\hline 40 & $4 / 26 / 82$ & 2230 & $38.39 .3^{\circ} \mathrm{N}$ & $72.25 .0^{\circ} \mathrm{W}$ & 13.2 & NP & 393 \\
\hline 47 & $4 / 20 / 82$ & 2330 & $38.43 .1^{\circ} \mathrm{N}$ & $72.11 .5^{\circ} \mathrm{W}$ & 15.8 & 311 & 42 \\
\hline 48 & $N G$ & & & & & & \\
\hline 49 & $4 / 27 / 82$ & $0 \cup 30^{\circ}$ & $38.46 .9^{\circ} \mathrm{N}$ & $71.58 .4^{\circ} \mathrm{W}$ & 165 & 415 & 521 \\
\hline bU & $4 / 27 / 82$ & 0130 & & $71.47 .0^{\circ} \mathrm{W}$ & 16.1 & & 555 \\
\hline b1 & $4 / 27 / 82$ & 0605 & $38.46 .4^{\circ} \mathrm{N}$ & $71.79 .2^{\circ} \mathrm{W}$ & 16.0 & 385 & 529 \\
\hline 52 & $4 / 27 / 82$ & 1115 & $38.38 .5^{\circ} \mathrm{N}$ & $71.45 .9^{\circ} \mathrm{W}$ & 15.9 & 321 & 503 \\
\hline 53 & $4 / 27 / 82$ & 1345 & $38.43 .1^{\circ} \mathrm{N}$ & $71.42 .9^{\circ} \mathrm{W}$ & 16.2 & 373 & 535 \\
\hline 54 & $4 / 27 / 82$ & 1445 & $38.50 .1^{\circ} \mathrm{N}$ & $71.38 .0^{\circ} \mathrm{W}$ & 16.2 & 370 & 530 \\
\hline 55 & $4 / 27 / 82$ & 1835 & $38.49 .6^{\circ} \mathrm{N}$ & $71.46 .0^{\circ} \mathrm{W}$ & 16.1 & 423 & 545 \\
\hline 56 & $4 / 28 / 82$ & 0133 & $38.51 .8^{\circ} \mathrm{N}$ & $71.40 .8^{\circ} \mathrm{W}$ & 16.1 & 413 & 550 \\
\hline 57 & $4 / 28 / 82$ & 0035 & $38.48 .5^{\circ} \mathrm{N}$ & $71.45 .7^{\circ} \mathrm{W}$ & 15.9 & 405 & 538 \\
\hline 58 & $4 / 28 / 82$ & 1440 & $38.54 .8^{\circ} \mathrm{N}$ & $71.41 .3^{\circ} \mathrm{W}$ & 15.9 & 435 & 545 \\
\hline
\end{tabular}




\begin{tabular}{|c|c|c|c|c|c|c|c|}
\hline XET & Late & $\begin{array}{c}\text { Time } \\
\text { (Local) }\end{array}$ & Latitude & Longitude & $\begin{array}{l}\text { XBT } \\
\text { Surface } \\
\text { Temp }\left({ }^{\circ} \mathrm{C}\right)\end{array}$ & $\begin{array}{l}\text { Depth } \\
15\end{array}$ & ${ }_{10}$ \\
\hline $5 y$ & $4 / 28 / 82$ & 1836 & $38.57 .4^{\circ} \mathrm{N}$ & $71.39 .5^{\circ} \mathrm{W}$ & 16.0 & 411 & 535 \\
\hline 60 & $4 / 28 / 82$ & 2200 & $39.06 .4^{\circ} \mathrm{N}$ & $71.40 .0^{\circ} \mathrm{W}$ & 15.9 & 352 & 511 \\
\hline Gi & $4 / 28 / 82$ & 2300 & $39.13 .4^{\circ} \mathrm{N}$ & $\because \quad \therefore .9^{\circ} \mathrm{W}$ & 15.2 & 162 & 432 \\
\hline . & $4 / 29 / 82$ & UUOO & $39.20 .6^{\circ} \mathrm{N}$ & $\because .94 .1^{\circ} \mathrm{W}$ & 13.8 & NP & 376 \\
\hline 政 & $4 / 29 / 82$ & 0113 & $39.29 .4^{\circ} \mathrm{N}$ & $\therefore .97 .8^{\circ} \mathrm{W}$ & 12.5 & NP & 269 \\
\hline 0 & $4 / 29 / 82$ & 0215 & $39.36 .4^{\circ} \mathrm{N}$ & ii.51.4 $4^{\circ} \mathrm{W}$ & 9.2 & NP & $20 ; 42 ; 62$ \\
\hline 05 & $4 ! 29 / 82$ & 0315 & $39.28 .9^{\circ} \mathrm{N}$ & 71.5 & 8.9 & NP & $85 ; 215$ \\
\hline$v$ & $4 / 29 / 82$ & 0415 & $39.24 .1^{\circ} \mathrm{N}$ & $71.57 .1^{\circ} \mathrm{W}$ & 9.9 & NP & $12 ; 303$ \\
\hline 67 & $4 / 29 / 82$ & 0558 & $39.20 .5^{\circ} \mathrm{N}$ & $71.55 .0^{\circ} \mathrm{W}$ & 12.9 & NP & 353 \\
\hline br & $4 / 29 / 82$ & 1718 & $39.22 .3^{\circ} \mathrm{N}$ & $71.39 .4^{\circ} \mathrm{W}$ & 13.9 & NP & 340 \\
\hline 6 & $4 / 30 / 82$ & 0410 & $39.19 .1^{\circ} \mathrm{N}$ & $71.28 .1^{\circ} \mathrm{W}$ & 14.9 & NP & 392 \\
\hline 70 & $4 / 30 / 82$ & 0506 & $39.09 .8^{\circ} \mathrm{N}$ & $71.31 .6^{\circ} \mathrm{W}$ & 15.5 & 210 & 465 \\
\hline 71 & $4 / 30 / 82$ & 0600 & $38.59 .7^{\circ} \mathrm{N}$ & $71.36 .0^{\circ} \mathrm{W}$ & 15.9 & 381 & 530 \\
\hline 72 & $4 / 30 / 82$ & 1147 & $38.50 .0^{\circ} \mathrm{N}$ & 71.49 & 15.9 & 360 & 470 \\
\hline 73 & $4 / 30 / 82$ & 1257 & $38.55 .6^{\circ} \mathrm{N}$ & 71.39 & 16.6 & 431 & 543 \\
\hline 74 & $5 / 01 / 82$ & U:244 & $38.51 .0^{\circ} \mathrm{N}$ & 71.53 & 16.7 & 420 & 745 \\
\hline 75 & $5 / 01 / 82$ & 0630 & $38.52 .2^{\circ} \mathrm{N}$ & $71.54 .7^{\circ} \mathrm{W}$ & 15.8 & 410 & 525 \\
\hline 70 & $5 / 01 / 82$ & 1048 & $38.55 .5^{\circ} \mathrm{N}$ & $71.59 .9^{\circ} \mathrm{W}$ & 15.9 & 370 & 526 \\
\hline 77 & $5 / 01 / 82$ & 1802 & $39.04 .5^{\circ} \mathrm{N}$ & $71.48 .3^{\circ} \mathrm{W}$ & 15.7 & 351 & 508 \\
\hline 78 & $5 / 01 / 82$ & 1900 & $39.12 .7^{\circ} \mathrm{N}$ & $71.36 .9^{\circ} \mathrm{W}$ & 15.0 & 155 & 400 \\
\hline 79 & $5 / 01 / 82$ & 2000 & $39.23 .0^{\circ} \mathrm{N}$ & $71.25 .8^{\circ} \mathrm{W}$ & 9.2 & NP & $45 ; 270$ \\
\hline 80 & $5 / 01 / 82$ & 2103 & $39.27 .0^{\circ} \mathrm{N}$ & $71.13 .3^{\circ} \mathrm{W}$ & 9.5 & NP & $55 ; 248$ \\
\hline 81 & $5 / 02 / 82$ & 0312 & $39.29 .4^{\circ} \mathrm{N}$ & $70.58 .8^{\circ} \mathrm{W}$ & 10.3 & NP & 255 \\
\hline 82 & $5 / 02 / 82$ & 0548 & $39.30 .3^{\circ} \mathrm{N}$ & $70.56 .4^{\circ} \mathrm{W}$ & 8.6 & NP & $15 ; 240$ \\
\hline 83 & $5 / 02 / 82$ & 1342 & $39.33 .5^{\circ} \mathrm{N}$ & $70.53 .1^{\circ} \mathrm{W}$ & 9.0 & NP & $32 ; 235$ \\
\hline 84 & $5 / 02 / 82$ & 1948 & $39.29 .7^{\circ} \mathrm{N}$ & $70.56 .3^{\circ} \mathrm{W}$ & 12.0 & NP & \\
\hline 85 & $5 / 03 / 82$ & 0215 & $39.40 .6^{\circ} \mathrm{N}$ & $70.48 .2^{\circ} \mathrm{W}$ & 9.7 & NP & $40 ; 2$ \\
\hline 86 & $5 / 03 / 82$ & 0300 & $39.49 .8^{\circ} \mathrm{N}$ & $70.48 .4^{\circ} \mathrm{W}$ & 8.7 & NP & $8 ; 20$ \\
\hline
\end{tabular}

$N P=$ Not present

$\mathrm{iVG}=$ Probe no good 